\title{
17. GEOCHEMICAL INVESTIGATIONS OF VOLCANIC ASH LAYERS FROM LEG 119, KERGUELEN PLATEAU 1
}

\author{
Wolfgang Morche, ${ }^{2}$ Hans-W. Hubberten, ${ }^{2}$ Werner U. Ehrmann, ${ }^{2}$ and Jörg Keller ${ }^{3}$
}

\begin{abstract}
Geochemical investigations were carried out on 19 discrete ash layers and on 42 dispersed ash accumulations in Oligocene to Pleistocene sediments from Sites 736, 737, 745, and 746 of ODP Leg 119 (Kerguelen Plateau in the southern Indian Ocean). The chemical data obtained from more than 500 single-grain glass analyses allow the characterization of two dominant petrographic rock series. The first consists of transitional- to alkali-basalts, the second mainly of trachytes with subordinated alkali-rhyolites and rhyolites. Chemical correlation with possible source areas indicates that the tephra layers from the northern Kerguelen Plateau Sites 736 and 737 were probably erupted from the nearby Kerguelen Islands. The investigated ash layers clearly reflect the Oligocene to recent changes in the composition of the volcanic material recorded from the Kerguelen Islands. The dispersed ashes from Sites 745 and 746 in the Australian-Antarctic Basin display almost the same range in chemical compositions as those from the north. Heard Island and other sources may have contributed to their formation, in addition to the Kerguelen Islands. Dispersed ash of calc-alkaline composition is most probably derived from the South Sandwich island arc, indicating sea-ice rafting as an important mechanism of transport.
\end{abstract}

\section{INTRODUCTION}

During Leg 119 of the Ocean Drilling Program (ODP) two sites (736 and 737) on the northern Kerguelen Plateau, two sites (738 and 744) on the southern Kerguelen Plateau, and two sites (745 and 746) in the Australian-Antarctic Basin were drilled (Barron, Larsen, et al., 1989; Fig. 1). The Kerguelen Plateau is situated between $46^{\circ}$ and $64^{\circ} \mathrm{S}$ in the southern Indian Ocean and trends northwest-southeast for approximately $2500 \mathrm{~km}$. It is some $500 \mathrm{~km}$ wide and rises 2-4 km above the adjacent deep-sea basins. The Kerguelen Plateau is the world's largest submarine plateau and can be subdivided into two distinct domains (Schlich, 1975; Houtz et al., 1977), the northern Kerguelen Plateau, or Kerguelen-Heard Plateau, located between $46^{\circ}$ and $54^{\circ} \mathrm{S}$, and the southern Kerguelen Plateau.

For our study of volcanic ash geochemistry, Sites 736 and 737 from the northern Kerguelen Plateau and Sites 745 and 746 from the Australian-Antarctic Basin just east of the southern Kerguelen Plateau were selected. At all of these sites, especially from the northern ones, volcanic ash material was encountered. It either was dispersed within the sediments or occurred as discrete ash layers, and reflects the influence of numerous volcanic eruptions through time. In contrast, Sites 738 and 744 on the southern tip of the plateau did not yield any ash layers or larger amounts of dispersed glass grains.

The purpose of this study was to determine the chemical compositions of the ash layers and with these data discuss the provenance of the volcanic material. Because of the prevailing westerly winds and eastward-flowing ocean surface currents in this region, source areas of tephras distributed by wind, ocean currents, and ice rafting are to be sought to the west of the drill sites. During the stratigraphic interval examined (Oligocene to the present), subaerial volcanic activity in the vicinity of the

\footnotetext{
I Barron, J., Larsen, B., et al., 1991. Proc. ODP, Sci. Results, 119: College Station, TX (Ocean Drilling Program).

${ }^{2}$ Alfred Wegener Institute for Polar and Marine Research, Columbusstraße, D-2850 Bremerhaven, Federal Republic of Germany.

${ }^{3}$ Mineralogisch-Petrographisches Institut, Universität Freiburg/Br., Albertstraße 23b, D-7800 Freiburg, Federal Republic of Germany.
}

drill sites is recorded from the Kerguelen Islands, Crozet Island, and Heard Island (Girod and Nougier, 1972).

The magmas erupted on the Kerguelen Islands display a wide compositional range (Fig. 2) characterized by two main rock series, an older tholeiitic to transitional basalt series that probably formed since Eocene time, which was followed by alkali basalts since Oligocene and a younger trachy-phonolitic series, which started in late Oligocene (Nougier, 1972a, 1972b; Watkins et al., 1974; Giret, 1983; Giret and Lameyre, 1983; Nougier et al., 1983; Giret et al., 1987). The volcanic products of Heard Island belong to both an alkali-basalt and a trachytic series, as on the Kerguelen Islands, and are presumed to have formed at least since late Miocene (Stephenson, 1972; Clarke et al., 1983). The volcanic rocks of Crozet Island, in contrast, are of one basaltic rock suite showing much less compositional variability (Chevallier et al., 1983; Gunn et al., 1972).

\section{MATERIAL}

For this study of volcanic ash geochemistry a total of 19 discrete ash layers from Holes 736A, 737A, 737B, and 745B were investigated. In addition, 42 dispersed ash concentrations from Holes 737B, 745B, and 746A were studied (Table 1).

Site $736\left(49^{\circ} 24.12^{\prime} \mathrm{S}, 71^{\circ} 39.61^{\prime} \mathrm{E} ; 629 \mathrm{~m}\right.$ water depth) penetrated to a total depth of $371 \mathrm{~m}$ below seafloor (mbsf). The oldest sediments recovered were late early Pliocene (Barron, Larsen, et al., 1989). Volcanic ash and pumice accumulations are restricted to the uppermost $50 \mathrm{~m}$ of Hole 736A and have a Quaternary age. All 12 discrete layers recovered were available for our investigations (Table 1 and Fig. 3). The samples from Cores $119-736 \mathrm{~A}-6 \mathrm{H}$ and $119-736 \mathrm{~A}-7 \mathrm{H}$, however, possibly may represent downfall material during coring.

We do not discuss here whether the ash and pumice layers are true layers of air-fall ash, were ice-rafted to the site, or represent so-called "disorganized turbidites," as debated for the coarsegrained basaltic debris by the shipboard scientific party (Barron, Larsen, et al., 1989).

Site 737 is situated about $100 \mathrm{~km}$ southeast of Site 736, at $50^{\circ} 13.67^{\prime} \mathrm{S}, 73^{\circ} 01.97^{\prime} \mathrm{E}$, and was drilled in a water depth of $564 \mathrm{~m}$. It penetrated to $715.5 \mathrm{mbsf}$ and recovered, beneath a few centimeters of Quaternary cover, lower Pliocene to middle Eocene sediments which complement the stratigraphic sequence re- 


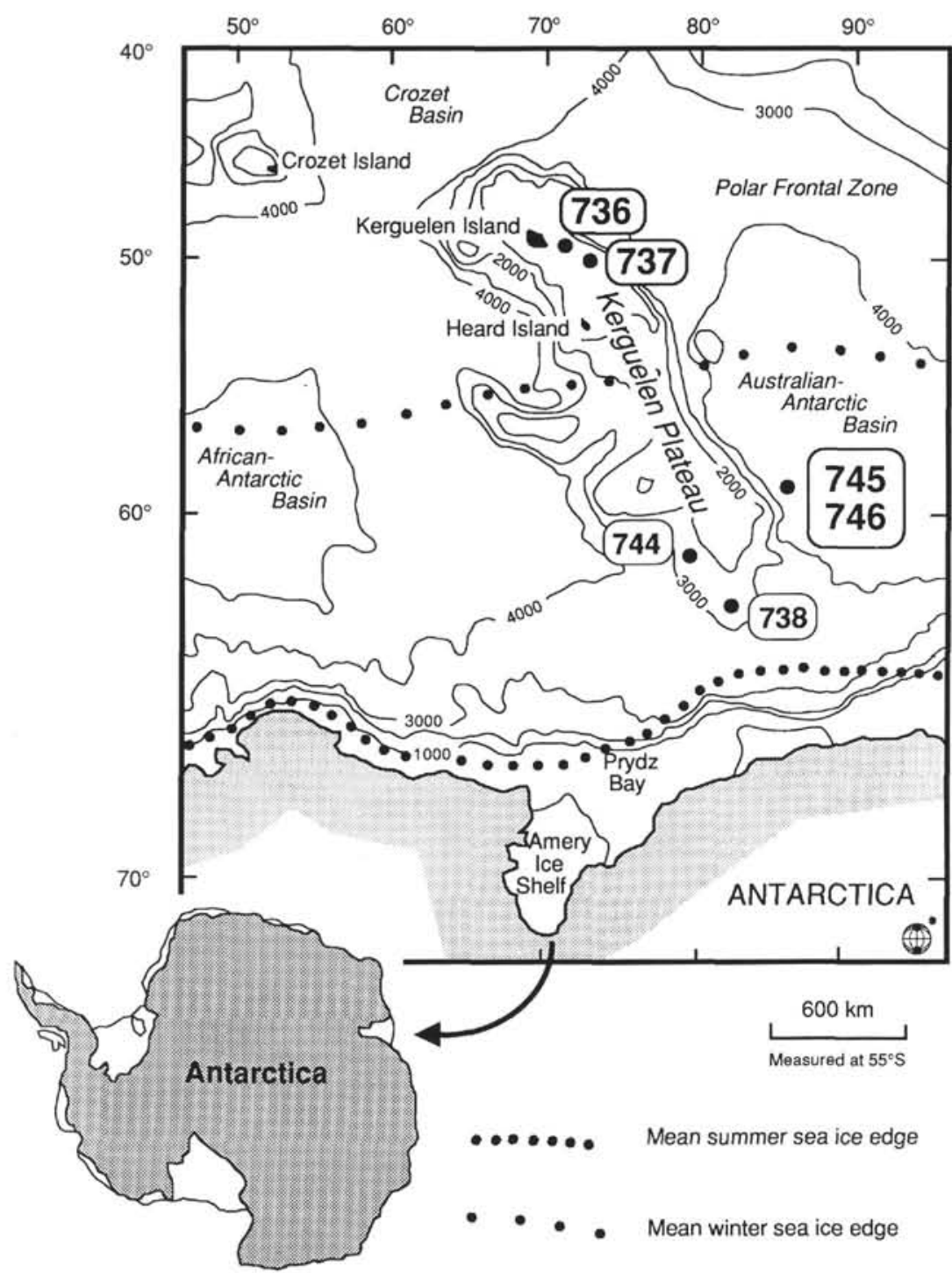

Figure 1. Location map of ODP Leg 119 drill sites in the southern Indian Ocean. Ash samples were investigated from Sites 736, 737, 745, and 746. Sites 738 and 744 were devoid of volcanic ash. Bathymetry (in meters) is from GEBCO (Hayes and Vogel, 1981; Fisher et al., 1982). The position of the Polar Front is according to Whitworth (1988), the sea-ice coverage according to Dietrich and Ulrich (1968).

covered at Site 736 (Barron, Larsen, et al., 1989). We described and sampled eight discrete ash layers from lower Pliocene to upper Oligocene sediments in Holes 737A and 737B (Table 1). The upper and lower Oligocene sediments (Cores 119-737B-13R to 119-737B-27R and 119-737B-33R to 119-737B-38R) are characterized by up to $10 \%$ dispersed volcanic glass (Barron, Larsen, et al., 1989). Therefore, in addition to the discrete ash layers, four samples of dispersed volcanic glass have been analyzed from the lower Oligocene strata (Table 1 and Fig. 3).

Site $745\left(59^{\circ} 35.71^{\prime} \mathrm{S}, 85^{\circ} 51.60^{\prime} \mathrm{E} ; 4308 \mathrm{~m}\right.$ water depth; 215 $\mathrm{m}$ penetration) and Site $746\left(59^{\circ} 32.82^{\prime} \mathrm{S}, 85^{\circ} 51.78^{\prime} \mathrm{E} ; 4070 \mathrm{~m}\right.$ water depth; $281 \mathrm{~m}$ penetration) together recovered a continuous upper Miocene to Quaternary sedimentary sequence (Barron, Larsen, et al., 1989). Only two discrete ash layers were obtained from Hole $745 \mathrm{~B}$, but glass shards were also recovered from samples, that had been systematically collected (one per core section; Ehrmann et al., this volume) for sedimentological studies (Table 1 and Fig. 3).

\section{METHODS}

All discrete ash layer samples were dried at $50^{\circ} \mathrm{C}$. Carbonate was removed by adding $10 \%$ hydrochloric acid. The carbonatefree residue was rinsed with distilled water and dried. The samples were then sieved in order to obtain the grain size fractions. Only the 63-125 $\mu \mathrm{m}$ and $125-250 \mu \mathrm{m}$ fractions were used for further investigations. From the dispersed ashes the sand-sized fraction $(>63 \mu \mathrm{m})$ of the carbonate-free and opal-free nonbiogenic material was studied. For convenience AWI laboratory numbers were used instead of full sample designation (Table 1).

The samples were first described using a petrographic microscope. Special textural phenomena were then documented with the help of a scanning electron microscope (SEM). The glass shards were separated using a heavy liquid and a Frantz Isodynamic magnetic separator, the magnetic field strength of which was varied in order to obtain a concentrated glass fraction and to get information on mineral contents. Polished grain mounts 


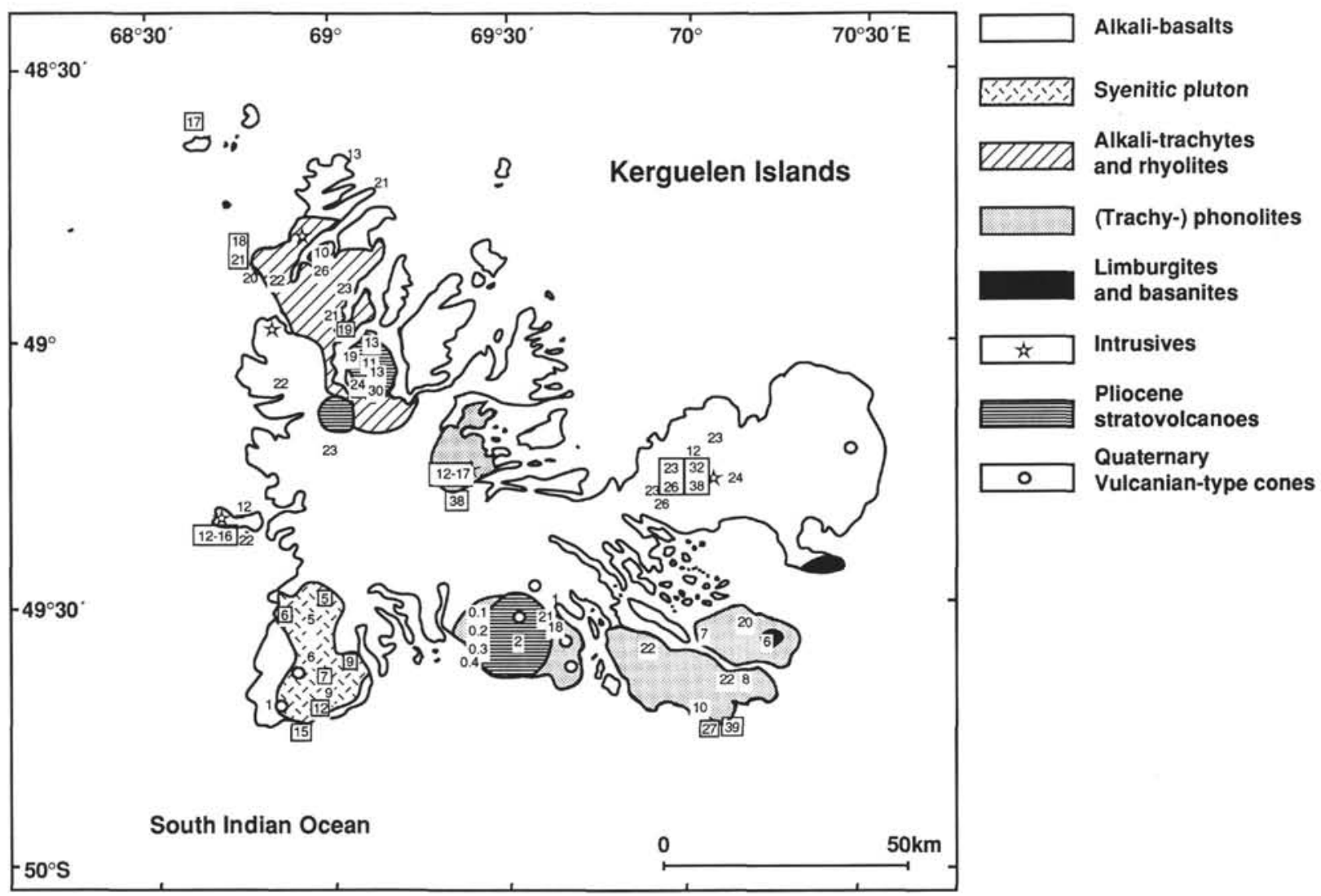

Figure 2. Schematic petrological map of the Kerguelen Islands, after Nougier (1972b). Compilation of age data from Giret et al. (1987): age data (in $\mathrm{Ma}$ ) in squares are related to plutons, those without squares to lava flows.

were prepared for microprobe analyses and petrographic investigations. The geochemical analyses were performed with energy dispersive KEVEX equipment (EDX) attached to an SEM at Freiburg University. To avoid uncontrolled loss of $\mathrm{Na}_{2} \mathrm{O}$, glass particles were analyzed under a defocused electron beam of 10 by $10 \mu \mathrm{m}$ size minimum (accelerating voltage $15 \mathrm{kV}$, beam current approximately $5 \mathrm{nA}$, counting time $100 \mathrm{~s}$ ). Matrix corrections and calibration with mineral and glass standards from the Smithsonian Institution (Jarosewich et al., 1980) were made. Selected analyses of standards are given in Table 2 for comparison.

\section{RESULTS}

The most important results of our study were obtained from the analyses of the discrete tephra layers from Holes 736A, 737A, and 737B. The ash and pumice layers of Hole 736A are Quaternary. The ash layers recovered from Holes 737A and 737B are early Oligocene to early Pliocene. Quaternary ashes could not be recovered at this site, because sediments of this age were only about $40 \mathrm{~cm}$ thick (Barron, Larsen, et al., 1989). No ash layers were recovered from Sites 738 and 744 on the southern Kerguelen Plateau, nor did the nonbiogenic $>63-\mu \mathrm{m}$ fraction of the samples from these sites contain significant amounts of volcanic glass.

\section{Petrography}

Maximum and median grain size of ash layers at Site 736 (mostly $>125 \mu \mathrm{m}$ ) is distinctly coarser than at Sites 737 and 745 (mostly $<125 \mu \mathrm{m}$; Fig. 4) and thus indicates that Site 736 is closer to the volcanic source, assuming similar modes of erup- tion and deposition. Content of heavy minerals (as indicated for example by content of ferromagnetics, Fig. 4) shows the same pattern and stresses the proximal position of Site 736. Quaternary tephras at Site 736 show a remarkable rounding of the clasts, thus indicating reworking of ash.

Most of the glass shards investigated are dark to pale brown or colorless, although some samples show bimodal composition with both the types. Glass textures range from dense, poorly vesicular, blocky shards to highly vesicular particles with an elongated, fibrous or platy bubble wall habit (Pl. 1.1 to 1.2$)$. The Quaternary tephras consist of fresh glass shards with almost no alteration visible under the SEM. In contrast, the Oligocene ashes from Hole 737B are heavily altered in part, and show etching cavities on the grain surfaces and coating by secondary minerals ( $\mathrm{Pl} .1 .3,1.4)$. Tiny mineral inclusions, such as $\mathrm{Fe}-\mathrm{Ti}$ oxides and pyroxenes, are common.

The mineral assemblages within the discrete ash layers (Table 3) are all thought to be phenocrysts (idiomorphic shape, adhering glass, etc.). The compositional difference between the glasses is also reflected in specific mineral assemblages. The Quaternary tephras from Hole 736A, which are composed almost exclusively of trachytes, are characterized by the occurrence of $\mathrm{Fe}-\mathrm{Ti}$ oxides, aegirine-augite, plagioclase, alkali feldspar, and apatite. The early Pliocene to Miocene tephras from Hole $737 \mathrm{~A}$ include the same mineralogy as at Hole $736 \mathrm{~A}$, commonly with additional contents of hedenbergite, biotite, and augite. The oldest Site 737 tephras, which are of Oligocene age, reveal alkali-basaltic to transitional basaltic composition and lack alkali pyroxenes and alkali feldspars. Plagioclase, augite, and, in two cases, olivine are the only phenocrysts. 
Table 1. List of samples taken from discrete tephra layers and samples containing dispersed ash material. ODP sample numbers and corresponding AWI laboratory numbers are given.

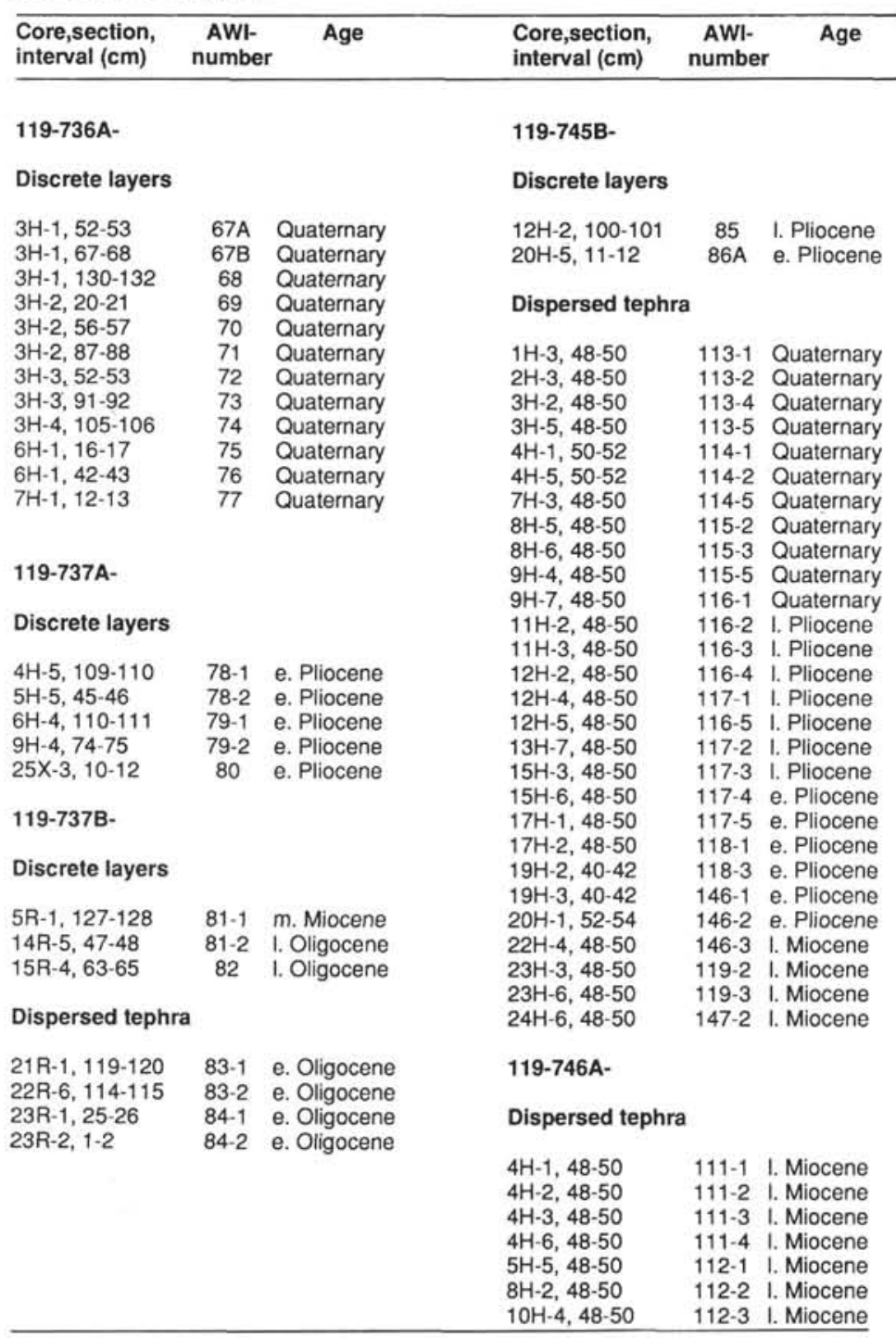

\section{Geochemistry}

The mean values of chemical data of about 500 analyses of individual glass shards from the Leg 119 tephra samples are presented in Table 4. The complete data set is listed in the Appendix.

The geochemistry of the discrete ash layers shows a large variation in most of the major elements, not only between, but also within different layers. Two distinctively different compositional groups can be recognized in the plots of total alkalis vs. silica (TAS diagrams, Fig. 5). The first group comprises all Quaternary ashes from Site 736, as well as the Miocene and early Pliocene ashes from Sites 737 and 745 . Most of these ashes are of trachytic composition (Le Bas et al., 1986), three of them showing an additional (alkali-) rhyolitic glass population. Widespread tephras of such composition are indicating strong explosive activity during these time intervals. The second group mainly comprises the Oligocene tephras of Hole 737B and displays a transitional- to alkali-basalt composition. The only other (basalt-andesitic to andesitic) tephra plotting within this group (Fig. 5, sample AWI 77), is from the Quaternary layers of Hole 736A. This ash layer includes a second glass population that is indistinguishable from the other trachytic Quaternary ashes of Hole 736A. Probably the two types of ash are from two discrete contemporaneous eruptive events, which have to be ascribed to different source areas.

The dispersed ashes from Sites 745 and 746 show almost the same geochemical trends as the total of the discrete ash layers from the other holes (Fig. 6). In Holes 745B and 746A the same compositional variability and the same two major groups (i.e., the transitional basalts to alkali basalts and the trachytic series) occur. However, subdivision into two groups, which is clearly related to age in the case of the discrete ash layers, does not seem to apply to the dispersed tephras. Basaltic shards, occurring almost exclusively in Oligocene sediments at the sites on the northern Kerguelen-Heard Plateau, are distributed throughout the late Miocene to Quaternary sediments of the southern sites. Additionally there is an input of dispersed basanitic ash over the whole time span recovered. Deposition of (alkali-) rhyolitic dispersed ash during late Miocene (AWI 111, 112, 146) can be 
NORTHERN KERGUELEN PLATEAU

Site 736

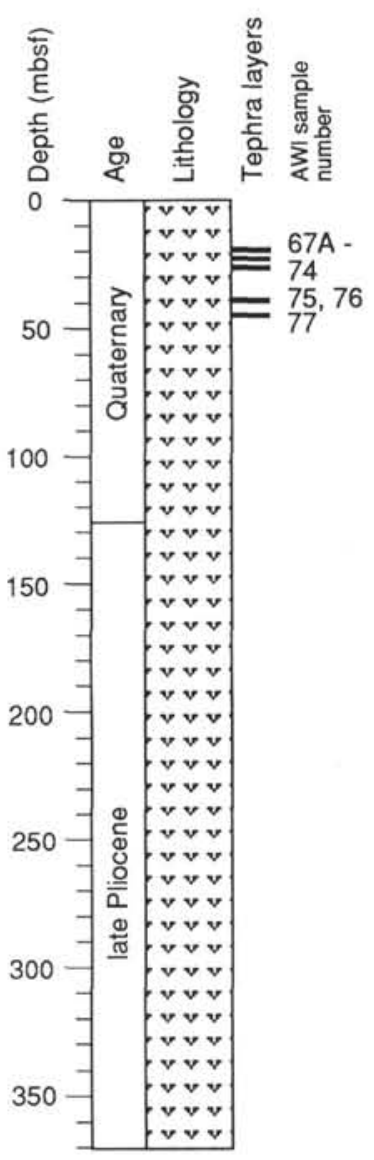

v v Diatom ooze

+.. Nannofossil

, o. oze

Calcareous

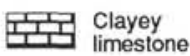
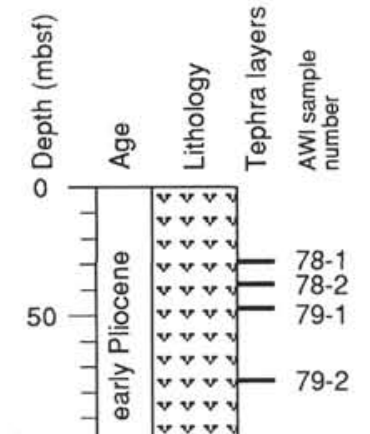

100

- 10.
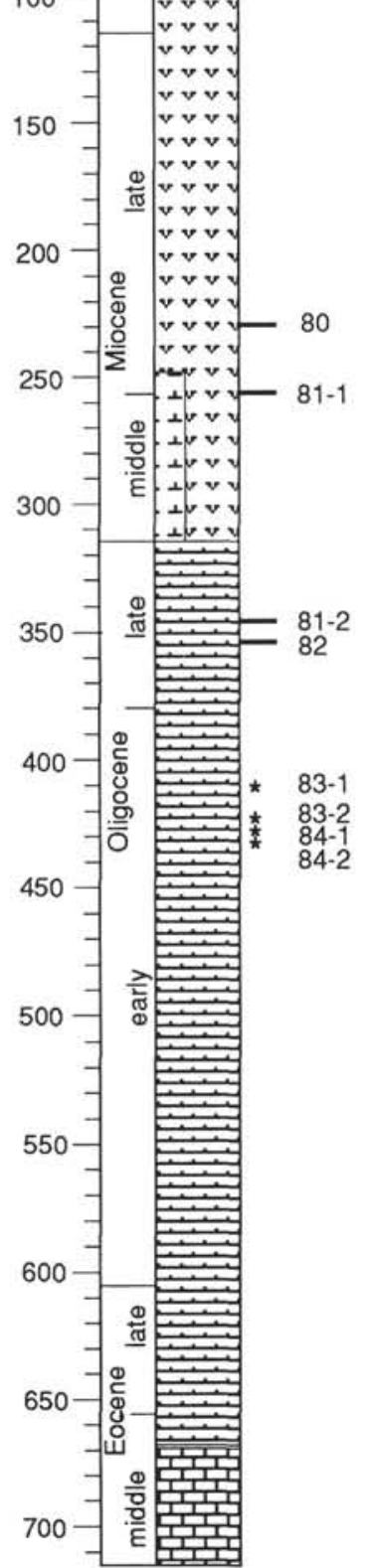

Site 745

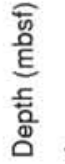

ठ্ㅎ 음

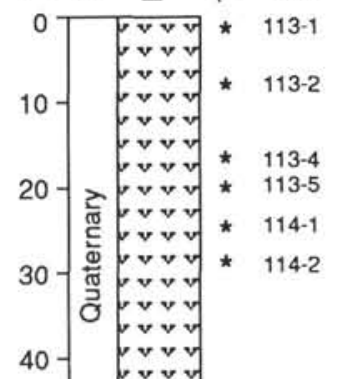

$50-\left[\begin{array}{lll}40-114-5 \\ v & v & v \\ v & v & v \\ v & v & v \\ v & v & v \\ v & v & v \\ v & v & v \\ v & v & v \\ v & v & v\end{array}\right] * 1$

$70-\left[\begin{array}{ll}v & v v \\ v & v v \\ v & v v \\ v & v v \\ v & v v\end{array} \mid \begin{array}{l}115-2 \\ 115-3\end{array}\right.$

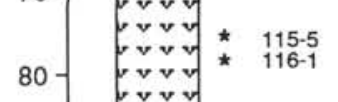

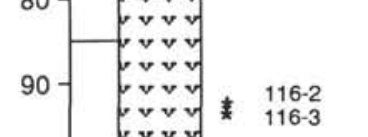

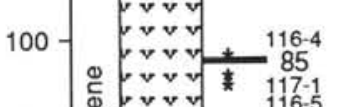

110

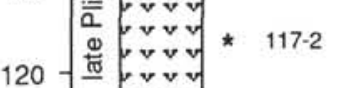

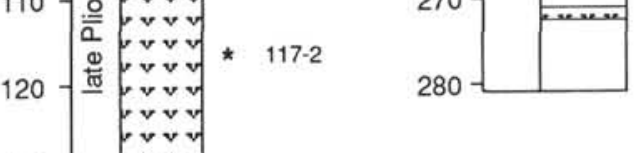

Site 746
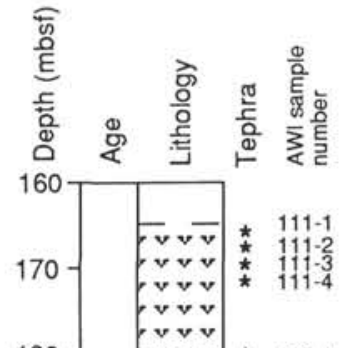

$180-\left(\begin{array}{l}v v v \\ v v v \\ v v v \\ v v v v\end{array}\right)+112-1$

$190-\left\{\begin{array}{l}v v v \\ v v v\end{array}\right.$

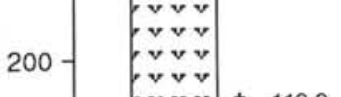

210
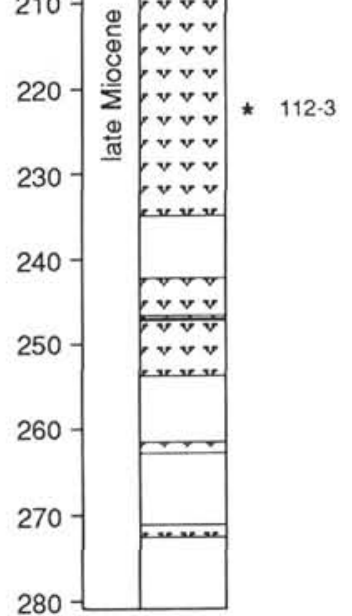

Figure 3. Schematic stratigraphic columns of ODP Sites 736, 737, 745, and 746. The position of the investigated discrete (solid line) and dispersed (asterisk) tephras and their AWI laboratory numbers are shown. 
Table 2. Analytical results obtained by EDX at Freiburg University on Smithsonian Institution reference glass and mineral standards. ${ }^{a}$

\begin{tabular}{|c|c|c|c|c|c|c|c|c|c|c|c|}
\hline USNM & & $(\%)$ & $\mathrm{SiO}_{2}$ & $\mathrm{TiO}_{2}$ & $\mathrm{Al}_{2} \mathrm{O}_{3}$ & $\mathrm{FeO}$ & $\mathrm{MnO}$ & $\mathrm{MgO}$ & $\mathrm{CaO}$ & $\mathrm{K}_{2} \mathrm{O}$ & $\mathrm{Na}_{2} \mathrm{O}$ \\
\hline 111240 & Smithsonian & (mean) & 51.11 & 1.86 & 14.14 & 11.91 & 0.22 & 6.75 & 11.18 & 0.19 & 2.64 \\
\hline Basalt & Freiburg & (mean) & 50.99 & 1.80 & 14.36 & 12.09 & 0.06 & 6.33 & 11.54 & 0.20 & 2.58 \\
\hline glass & & (s & 0.13 & 0.08 & 0.22 & 0.10 & 0.11 & 0.13 & 0.04 & 0.03 & 0.14 \\
\hline 113498 & Smithsonian & (mean) & 51.68 & 4.12 & 12.67 & 13.40 & 0.00 & 5.15 & 9.44 & 0.83 & 2.70 \\
\hline Basalt & Freiburg & (mean) & 51.71 & 3.95 & 12.92 & 13.80 & 0.13 & 4.34 & 9.54 & 0.89 & 2.70 \\
\hline glass & & (SD) & 0.17 & 0.06 & 0.17 & 0.21 & 0.08 & 0.14 & 0.09 & 0.03 & 0.10 \\
\hline 11371 & Smithsonian & $(\mathrm{m}$ & 5 & 31 & 1 & .12 & 00 & 8.26 & 39 & 0.09 & 2.49 \\
\hline Bas & Freiburg & (me & 51.93 & 1.25 & 15.62 & 9.34 & 0.03 & 7.46 & 11.60 & 0.08 & 2.51 \\
\hline glass & & & 0.54 & 0.09 & 0.25 & 0.13 & 0.07 & 0.32 & 0.14 & 0.05 & 0.14 \\
\hline \multirow{3}{*}{$\begin{array}{l}72854 \\
\text { Rhyolite } \\
\text { glass }\end{array}$} & Smith & (me & 77.19 & 0.12 & 12.14 & 1.24 & 0.03 & 0.09 & 0.50 & 4.92 & 3.77 \\
\hline & Freiburg & (mean) & 77.71 & 0.03 & 12.38 & 1.07 & 0.00 & 0.00 & 0.46 & 5.03 & 3.24 \\
\hline & & ISD & 0.23 & 0.07 & 0.05 & 0.06 & 0.00 & 0.00 & 0.05 & 0.03 & 0.08 \\
\hline \multirow{3}{*}{$\begin{array}{l}137041 \\
\text { Anorthite }\end{array}$} & Smithsonian & (mean) & 43.86 & 0.03 & 35.91 & 0.62 & 0.00 & 0.00 & 19.03 & 0.03 & 0.53 \\
\hline & Freiburg & (mean) & 44.35 & 0.00 & 35.71 & 0.32 & 0.00 & 0.00 & 19.58 & 0.00 & 0.00 \\
\hline & & (SD) & 0.11 & 0.00 & 0.18 & 0.05 & 0.00 & 0.00 & 0.13 & 0.00 & 0.00 \\
\hline \multirow{3}{*}{$\begin{array}{l}133868 \\
\text { Anorthoclas }\end{array}$} & Smithsonian & (mea & 66.92 & 0.00 & 20.26 & 0.2 & 0.00 & 0.00 & 0.88 & 2.37 & 9.38 \\
\hline & se Freiburg & (me & 67.55 & 0.00 & 20.27 & 0.1 & 0.00 & 0.00 & 0.60 & 2.54 & 8.94 \\
\hline & & & 0.25 & 0.00 & 0.11 & 0.11 & 0.00 & 0.00 & 0.06 & 0.20 & 0.12 \\
\hline
\end{tabular}

${ }^{a}$ Mean of Freiburg analyses are based on 4-8 single analyses ( $\mathrm{SD}=$ standard deviation). The given reference analyses (numbers = USNM standards) are taken from Jarosewich et al. (1980).

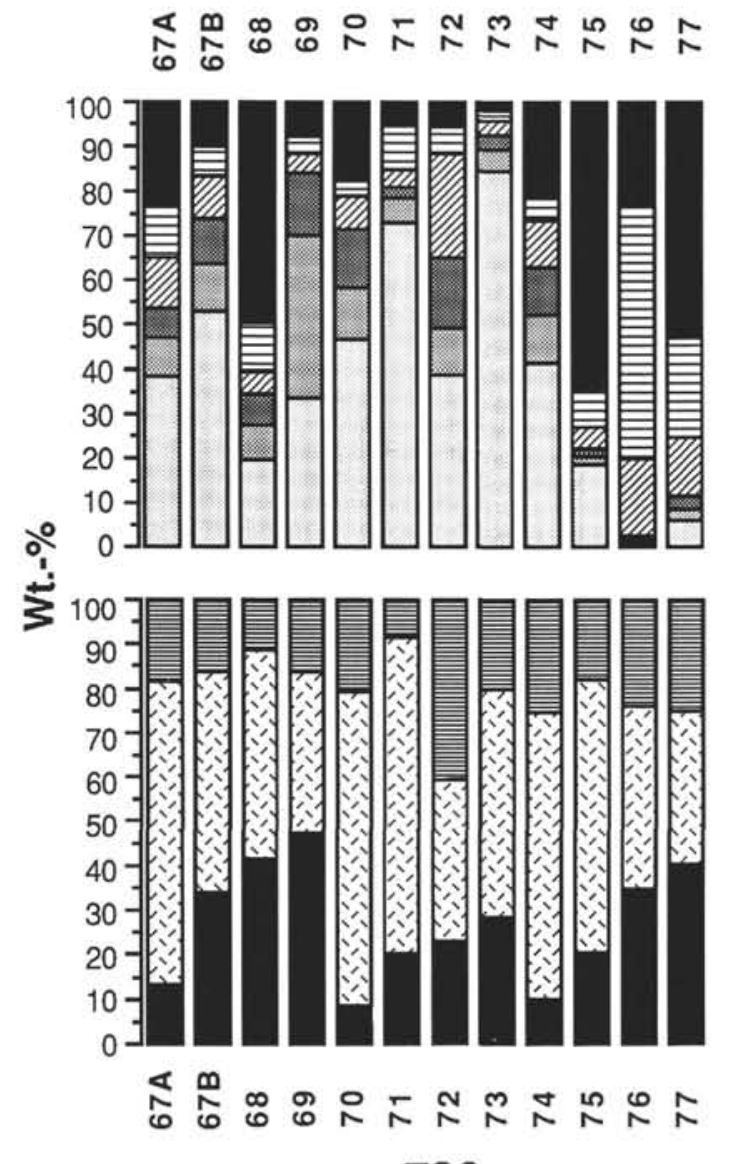

736
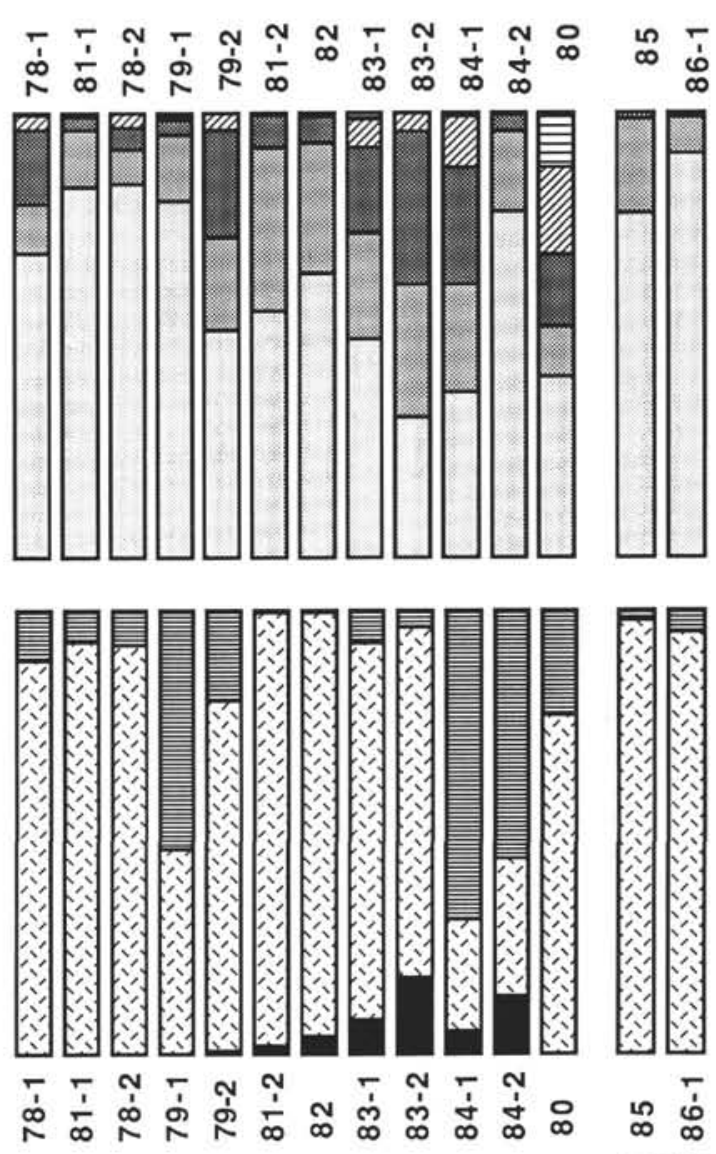

737
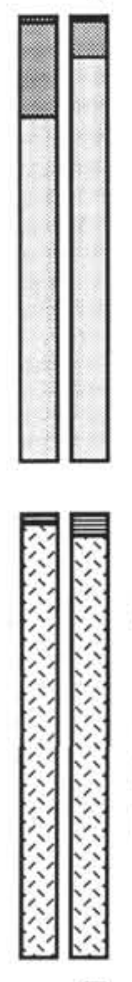

Grain size

$(\mu \mathrm{m})$

- $>1000$

目 500-1000

250-500

밈 $125-250$

63-125

$<63$

\section{Relative field strength of \\ magnetic separator (Ampere)}
目 $>1.2(\mathrm{Fsp}, \mathrm{Qz})$
[ $<1.2$ (Glass)
口 $<0.1$ (Mt)

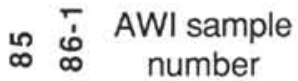 \\ 745 Site}

Figure 4. Results of grain size analysis and magnetic separating of tephra layers from Sites 736,737 , and 745.

correlated with the corresponding discrete tephra layer at Hole 737B (AWI 80), thus indicating strong explosive rhyolitic volcanism at this time.

At Site 745, furthermore, "exotic" glass shards of basalt-andesitic to andesitic composition occur during Quaternary to late
Pliocene (AWI 113-2/4, 115-3, 117-2, see Table 5) similar to the calc-alkaline Quaternary ash of Site 736 (AWI 77). All these tephras represent an island-arc tholeiitic series that is characteristic for the South Sandwich Islands about $6500 \mathrm{~km}$ to the west, thus indicating extensive sea-ice rafting as an important trans- 
Table 3. Petrographic summary of the ash samples studied. Mineral determination by petrographic microscope and EDXanalysis in part."

\begin{tabular}{|c|c|c|c|c|c|c|c|c|c|c|c|}
\hline $\begin{array}{l}\text { Core, section, } \\
\text { interval }(\mathrm{cm})\end{array}$ & $\begin{array}{c}\text { AWI- } \\
\text { number }\end{array}$ & Magnetite & Olivine & Augite & $\begin{array}{l}\text { Heden- } \\
\text { bergite }\end{array}$ & $\begin{array}{l}\text { Aegirine- } \\
\text { augite }\end{array}$ & Biotite & Plagioclase & $\begin{array}{l}\text { Alkali- } \\
\text { feldspar }\end{array}$ & Apatite & $\begin{array}{c}\text { Glass } \\
\text { Composition }\end{array}$ \\
\hline \multicolumn{12}{|l|}{$119.736 \mathrm{~A}=$} \\
\hline $3 \mathrm{H}-1,52-53$ & $67 \mathrm{~A}$ & + & & & & + & & + & + & + & $T$ \\
\hline $3 \mathrm{H}-1,67-68$ & $67 B$ & + & & & & + & & + & + & + & $\mathrm{T}$ \\
\hline $3 \mathrm{H}-1,130-132$ & 68 & + & & & & + & & + & + & + & $T$ \\
\hline $3 \mathrm{H}-2,20-021$ & 69 & + & & & & + & & + & + & + & $T / A B$ \\
\hline $3 \mathrm{H}-2,56-57$ & 70 & + & & & & ++ & & + & + & + & $\mathrm{T}$ \\
\hline $3 \mathrm{H}-2,87-88$ & 71 & + & & & & + & & + & + & + & $\mathrm{T}$ \\
\hline $3 \mathrm{H}-3,52 \cdot 53$ & 72 & + & & & & ++ & & + & + & + & $T$ \\
\hline $3 \mathrm{H}-3,91-92$ & 73 & + & & & & + & & + & + & + & T/BTA \\
\hline $3 \mathrm{H}-4,105-106$ & 74 & + & & & & ++ & & + & + & + & $T$ \\
\hline $6 \mathrm{H}-1,16-17$ & 75 & + & & & + & + & & + & + & + & $T$ \\
\hline $6 \mathrm{H}-1,42-43$ & 76 & + & & + & + & + & + & + & + & + & T \\
\hline $7 \mathrm{H}-1,12-13$ & 77 & + & & + & + & + & + & + & + & + & T/BTA/AND \\
\hline \multicolumn{12}{|l|}{$119 \cdot 737 \mathrm{~A}$. } \\
\hline $4 \mathrm{H}-5,109-110$ & $78-1$ & + & & & + & + & + & + & + & + & $T$ \\
\hline $5 \mathrm{H}-5,45-46$ & $78-2$ & + & & & + & + & + & + & + & + & $\mathrm{T}$ \\
\hline $6 \mathrm{H}-4,110-111$ & $79-1$ & + & & + & + & + & + & + & + & + & $\mathrm{T}$ \\
\hline $9 \mathrm{H}-4,74-75$ & $79-2$ & + & & + & + & + & + & + & + & + & T/AR \\
\hline $25 x-3,10-12$ & 80 & + & & & + & & & + & + & + & AR \\
\hline \multicolumn{12}{|l|}{$119-737 \mathrm{~B}}$. \\
\hline $5 R-1,127-128$ & $81-1$ & + & & + & + & & & + & + & + & $T$ \\
\hline $14 R-5,47-48$ & $81-2$ & + & & + & & & & + & & & AB/BAND \\
\hline $15 \mathrm{R} \cdot 4,63-65$ & 82 & + & & + & & & & + & & & TB/BAND \\
\hline $21 R-1,119-120$ & $83-1$ & + & & + & & & & + & & & TB/BAND \\
\hline $22 \mathrm{R}-6,114-115$ & $83-2$ & + & & + & & & & + & & & $A B$ \\
\hline $23 R-1,25-26$ & $84-1$ & + & + & & & & & + & & & $A B / T$ \\
\hline $23 R-2,1-2$ & $84-2$ & + & + & & & & & + & & & $\mathrm{AB}$ \\
\hline \multicolumn{12}{|l|}{$119-745 \mathrm{~B}$} \\
\hline $12 \mathrm{H}-2,100-101$ & 85 & + & & & + & + & & + & + & + & $T$ \\
\hline $20 \mathrm{H}-5,11-12$ & $86-1$ & + & & & + & + & & + & + & + & $T$ \\
\hline
\end{tabular}

port mechanism for volcanic ash. In Hole 701A of ODP Leg 114 close to the South Sandwich Islands several tephra layers of comparable composition have been found in Quaternary sediments (Hubberten et al., in press; Table 5).

\section{DISCUSSION}

Tephras at Sites 736 and 737 may be partly or entirely derived from the volcanoes of the Kerguelen Islands, $70 \mathrm{~km}$ and $150 \mathrm{~km}$ upwind from the two sites, respectively. Although the ash layers occurring in the sediments of both sites belong to different geologic ages, and therefore cannot be directly compared, the greater distance of Site 737 from the eruption center may be reflected in the smaller grain size of the volcanic material and minor content of heavy minerals (Fig. 4). Grain size distribution and low mineral content of the two discrete layers at Site $\mathbf{7 4 5}$ (ca. $1000 \mathrm{~km}$ downwind from the Kerguelen Islands) emphasize their more distal position.

The geochemical variations of the two ash groups encountered in the sediments of Sites 736,737 , and 745 show that both represent an almost complete compositional suite. When the composition of the analyzed glass shards is plotted together with published analyses of rocks from the Kerguelen Islands (Nougier, 1970) on a TAS diagram (Fig. 7), there is almost complete overlap. This is another indicator that the tephras probably derived from the Kerguelen Islands.

The subdivision of the tephras in an Oligocene basaltic group and a younger trachytic group should also be reflected in the eruptive sequence of the Kerguelen Islands (Fig. 8), if this is the eruption center. On the Kerguelen Islands, volcanic activity in late Oligocene to early Miocene time is dominated by the erup- tion of products of transitional- to alkali-basalt composition with subordinated eruptions of evolved magma (Nougier, 1972a; Watkins et al., 1974; Giret, 1983; Giret et al., 1987). This coincides well with the ash compositions found in the Oligocene section of Hole 737B (AWI 81-2 to 84-2).

The Miocene, Pliocene, and Quaternary tephras of Sites 736, 737 , and 745 belong predominantly to the trachytic series. As volcanic rocks displaying trachytic composition have erupted at the Kerguelen Islands during the Miocene to Quaternary time span (Nougier, 1972a, 1972b; Watkins et al. 1974; Giret et al., 1987), we suggest that the Kerguelen Islands also can be regarded as the source of the younger ashes found at these sites.

Other possible sources of the tephras could be Heard Island, about $450 \mathrm{~km}$ southeast of the Kerguelen Islands, and Crozet Island in the northwest. Heard Island shows many similarities with Kerguelen in terms of the composition of the magmatic rock series (Stephenson, 1972; Clarke et al., 1983). According to the position of Heard Island downwind of the Sites 736 and 737 , it can be regarded as a source area only for tephras at Sites 745 and 746 . The volcanoes of Crozet Island are Quaternary alkali-basalt (Gunn et al., 1972; Chevallier et al., 1983) and therefore cannot be related to the Oligocene basaltic or Quaternary trachytic tephras found.

It is therefore concluded that almost all of the discrete tephra layers studied at these sites were erupted from the Kerguelen Islands. A preliminary and schematic geochronological synopsis, comparing the tephra layers with the rock series of the Kerguelen Islands, is shown in Figure 8.

The provenance of the dispersed ash material from Sites 745 and 746 is much more difficult to define. These ashes show in 
Table 4. Mean values of chemical data from the ash layers studied. For ash layers which contain different populations of glass shards, these are indicated as (A), (B,) and $(C)$, respectively. ${ }^{a}$

\begin{tabular}{|c|c|c|c|c|c|c|c|c|c|c|c|c|}
\hline \multicolumn{2}{|l|}{$\begin{array}{l}\text { AWl- } \\
\text { number }\end{array}$} & $\begin{array}{c}\mathrm{P}_{2} \mathrm{O}_{5} \\
(\%)\end{array}$ & $\begin{array}{c}\mathrm{SiO}_{2} \\
(\%)\end{array}$ & $\begin{array}{l}\mathrm{TiO}_{2} \\
(\%)\end{array}$ & $\begin{array}{c}\mathrm{Al}_{2} \mathrm{O}_{3} \\
(\%)\end{array}$ & $\begin{array}{l}\mathrm{FeO} \\
(\%)\end{array}$ & $\begin{array}{c}\text { MnO } \\
(\%)\end{array}$ & $\begin{array}{l}\mathrm{MgO} \\
(\%)\end{array}$ & $\begin{array}{l}\mathrm{CaO} \\
(\%)\end{array}$ & $\begin{array}{l}\mathrm{K}_{2} \mathrm{O} \\
(\%)\end{array}$ & $\begin{array}{c}\mathrm{Na}_{2} \mathrm{O} \\
(\%)\end{array}$ & $\begin{array}{l}\mathrm{Cl} \\
(\%)\end{array}$ \\
\hline \multicolumn{13}{|c|}{ Hole 736A, discrete } \\
\hline 7-A & $\begin{array}{l}\text { Mean } \\
\text { SD }\end{array}$ & $\begin{array}{l}0.00 \\
0.00\end{array}$ & $\begin{array}{l}64.62 \\
1.90\end{array}$ & $\begin{array}{l}0.32 \\
0.13\end{array}$ & $\begin{array}{l}16.62 \\
1.54\end{array}$ & $\begin{array}{l}4.58 \\
0.69\end{array}$ & $\begin{array}{l}0.05 \\
0.09\end{array}$ & $\begin{array}{l}0.00 \\
0.00\end{array}$ & $\begin{array}{l}1.26 \\
0.40\end{array}$ & $\begin{array}{l}5.47 \\
0.46\end{array}$ & $\begin{array}{l}6.83 \\
0.49\end{array}$ & $\begin{array}{l}0.23 \\
0.09\end{array}$ \\
\hline $7 . B$ & $\begin{array}{l}\text { Mean } \\
\text { SD }\end{array}$ & $\begin{array}{l}0.00 \\
0.00\end{array}$ & $\begin{array}{l}4.72 \\
01\end{array}$ & $\begin{array}{l}0.36 \\
0.13\end{array}$ & $\begin{array}{l}16.72 \\
1.76\end{array}$ & $\begin{array}{l}4.36 \\
0.56\end{array}$ & $\begin{array}{l}0.03 \\
0.07\end{array}$ & $\begin{array}{l}0.00 \\
0.00\end{array}$ & $\begin{array}{l}1.17 \\
0.40\end{array}$ & $\begin{array}{l}61 \\
.63\end{array}$ & $\begin{array}{l}6.80 \\
0.60\end{array}$ & $\begin{array}{l}0.22 \\
0.13\end{array}$ \\
\hline 68 & $\begin{array}{l}\text { Mean } \\
\text { SD }\end{array}$ & $\begin{array}{l}0.00 \\
0.00\end{array}$ & $\begin{array}{l}62.26 \\
1.94\end{array}$ & $\begin{array}{l}0.35 \\
0.14\end{array}$ & & $\begin{array}{l}4.68 \\
0.75\end{array}$ & $\begin{array}{l}0.00 \\
0.00\end{array}$ & $\begin{array}{l}0.00 \\
0.00\end{array}$ & $\begin{array}{l}1.93 \\
0.54\end{array}$ & $\begin{array}{l}5.93 \\
0.37\end{array}$ & $\begin{array}{l}6.39 \\
0.46\end{array}$ & $\begin{array}{l}0.16 \\
0.05\end{array}$ \\
\hline $69(A)$ & $n=1$ & 0.78 & 51.20 & 3.17 & 15.65 & 8.01 & 0.00 & 6.11 & 9.45 & 1.64 & 3.99 & 0.00 \\
\hline $9(\mathrm{~B})$ & $\begin{array}{l}\text { Mean } \\
\text { SD }\end{array}$ & $\begin{array}{l}0.00 \\
0.00\end{array}$ & $\begin{array}{l}65.32 \\
0.76\end{array}$ & $\begin{array}{l}0.39 \\
0.22\end{array}$ & & $\begin{array}{l}3.54 \\
0.21\end{array}$ & $\begin{array}{l}0.00 \\
0.00\end{array}$ & $\begin{array}{l}0.00 \\
0.00\end{array}$ & $\begin{array}{l}1.18 \\
0.46\end{array}$ & $\begin{array}{l}5.76 \\
0.92\end{array}$ & $\begin{array}{l}6.39 \\
1.16\end{array}$ & $\begin{array}{l}0.13 \\
0.10\end{array}$ \\
\hline 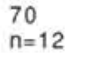 & ean & $\begin{array}{l}0.00 \\
0.00\end{array}$ & & & & $\begin{array}{l}3.32 \\
0.75\end{array}$ & $\begin{array}{l}.00 \\
.00\end{array}$ & $\begin{array}{l}0.00 \\
0.00\end{array}$ & $\begin{array}{l}1.45 \\
0.39\end{array}$ & $\begin{array}{l}.31 \\
48\end{array}$ & $\begin{array}{l}7.62 \\
0.75\end{array}$ & $\begin{array}{l}0.22 \\
0.09\end{array}$ \\
\hline 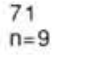 & an & & & & & $\begin{array}{l}5.62 \\
0.85\end{array}$ & $\begin{array}{l}.02 \\
.05\end{array}$ & $\begin{array}{l}0.00 \\
0.00\end{array}$ & $\begin{array}{l}2.26 \\
0.15\end{array}$ & $\begin{array}{l}94 \\
17\end{array}$ & $\begin{array}{l}6.51 \\
0.35\end{array}$ & $\begin{array}{l}0.13 \\
0.04\end{array}$ \\
\hline 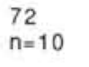 & $\begin{array}{l}\text { Mean } \\
\text { SD }\end{array}$ & & & $\begin{array}{l}34 \\
16\end{array}$ & & $\begin{array}{l}4.43 \\
0.38\end{array}$ & $\begin{array}{l}.00 \\
.00\end{array}$ & $\begin{array}{l}0.00 \\
0.00\end{array}$ & $\begin{array}{l}1.17 \\
0.25\end{array}$ & $\begin{array}{l}92 \\
41\end{array}$ & $\begin{array}{l}6.22 \\
0.23\end{array}$ & $\begin{array}{l}0.17 \\
0.05\end{array}$ \\
\hline 12 & an & $\begin{array}{l}0 \\
0\end{array}$ & & & & 79 & $\begin{array}{l}3 \\
6\end{array}$ & & $\begin{array}{l}1.75 \\
1.39\end{array}$ & $\begin{array}{l}37 \\
57\end{array}$ & $\begin{array}{l}51 \\
02\end{array}$ & $\begin{array}{l}0.24 \\
0.08\end{array}$ \\
\hline 4 & $\begin{array}{l}\text { Mean } \\
\text { SD }\end{array}$ & $\begin{array}{l}0.00 \\
0.00\end{array}$ & & $\begin{array}{l}0.52 \\
0.22\end{array}$ & & $\begin{array}{l}3.82 \\
0.52\end{array}$ & $\begin{array}{l}.02 \\
.06\end{array}$ & $\begin{array}{l}0.00 \\
0.00\end{array}$ & $\begin{array}{l}0.80 \\
0.20\end{array}$ & $\begin{array}{l}58 \\
40\end{array}$ & $\begin{array}{l}.50 \\
.45\end{array}$ & $\begin{array}{l}0.18 \\
0.11\end{array}$ \\
\hline$=10$ & $\begin{array}{l}\text { Mean } \\
\text { SD }\end{array}$ & $\begin{array}{l}0.00 \\
0.00\end{array}$ & 7 & $\begin{array}{l}0.43 \\
0.08\end{array}$ & $\begin{array}{l}15.95 \\
0.85\end{array}$ & $\begin{array}{l}3.99 \\
1.03\end{array}$ & $\begin{array}{l}.01 \\
.04\end{array}$ & $\begin{array}{l}0.00 \\
0.00\end{array}$ & $\begin{array}{l}0.89 \\
0.26\end{array}$ & $\begin{array}{l}66 \\
33\end{array}$ & $\begin{array}{l}6.49 \\
0.35\end{array}$ & $\begin{array}{l}0.22 \\
0.07\end{array}$ \\
\hline$=10$ & $\begin{array}{l}\text { Mean } \\
\text { SD }\end{array}$ & $\begin{array}{l}0.00 \\
0.00\end{array}$ & $\begin{array}{l}5.20 \\
.77\end{array}$ & $\begin{array}{l}0.47 \\
0.19\end{array}$ & $\begin{array}{l}16 \\
1.2\end{array}$ & $\begin{array}{l}4.27 \\
0.71\end{array}$ & $\begin{array}{l}0.03 \\
0.07\end{array}$ & $\begin{array}{l}0.00 \\
0.00\end{array}$ & $\begin{array}{l}1.11 \\
0.30\end{array}$ & $\begin{array}{l}.86 \\
61\end{array}$ & $\begin{array}{l}6.42 \\
0.78\end{array}$ & $\begin{array}{l}0.20 \\
0.11\end{array}$ \\
\hline $77(\mathrm{~A})$ & $n=1$ & 0.70 & 52.54 & 2.89 & 14.38 & 12.39 & 0.30 & 2.80 & 7.60 & 2.07 & 4.22 & 0.10 \\
\hline $\begin{array}{l}77(1) \\
n=7\end{array}$ & $\begin{array}{l}\text { Mean } \\
\text { SD }\end{array}$ & $\begin{array}{l}0.00 \\
0.00\end{array}$ & 8 & $\begin{array}{l}1.09 \\
0.15\end{array}$ & $\begin{array}{l}15 . \\
1.1\end{array}$ & $\begin{array}{l}10.69 \\
2.06\end{array}$ & $\begin{array}{l}0.09 \\
0.09\end{array}$ & $\begin{array}{l}2.91 \\
1.29\end{array}$ & $\begin{array}{l}8.74 \\
1.11\end{array}$ & $\begin{array}{l}0.50 \\
0.10\end{array}$ & $\begin{array}{l}2.98 \\
0.57\end{array}$ & $\begin{array}{l}0.08 \\
0.07\end{array}$ \\
\hline $77(\mathrm{C})$ & $\begin{array}{l}\text { Mean } \\
\text { SD }\end{array}$ & $\begin{array}{l}0.00 \\
0.00\end{array}$ & $\begin{array}{l}65.40 \\
1.57\end{array}$ & $\begin{array}{l}0.49 \\
0.11\end{array}$ & $\begin{array}{l}16.25 \\
1.11\end{array}$ & $\begin{array}{l}4.44 \\
0.87\end{array}$ & $\begin{array}{l}0.04 \\
0.08\end{array}$ & $\begin{array}{l}0.00 \\
0.00\end{array}$ & $\begin{array}{l}1.02 \\
0.48\end{array}$ & $\begin{array}{l}5.76 \\
0.57\end{array}$ & $\begin{array}{l}6.47 \\
0.71\end{array}$ & $\begin{array}{l}0.13 \\
0.11\end{array}$ \\
\hline
\end{tabular}

\section{Hole 737A, discrete ash layers}

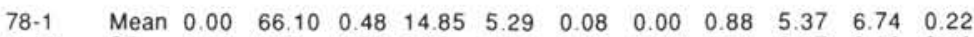
$\mathrm{n}=11 \quad \begin{array}{lllllllllllll} & \text { SD } & 0.00 & 0.91 & 0.15 & 1.35 & 0.64 & 0.09 & 0.00 & 0.39 & 0.67 & 0.90 & 0.10\end{array}$

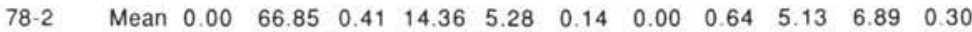
$\begin{array}{lllllllllllll}n=10 & \text { SD } & 0.00 & 0.31 & 0.06 & 0.33 & 0.14 & 0.08 & 0.00 & 0.06 & 0.17 & 0.23 & 0.05\end{array}$

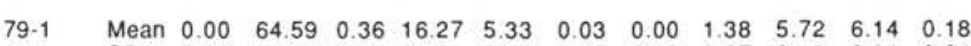

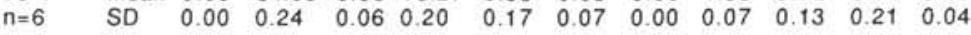

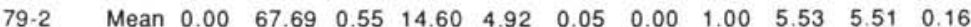
$\mathrm{n}=12 \quad \begin{array}{llllllllllll}\text { SD } & 0.00 & 1.73 & 0.19 & 1.05 & 0.30 & 0.09 & 0.00 & 0.41 & 0.18 & 0.44 & 0.08\end{array}$

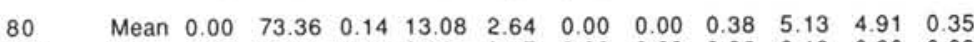
$\begin{array}{lllllllllllll}\mathrm{n}=9 & \mathrm{SD} & 0.00 & 0.62 & 0.08 & 0.32 & 0.17 & 0.00 & 0.00 & 0.06 & 0.19 & 0.30 & 0.03\end{array}$

\section{Hole $737 \mathrm{~B}$, discrete ash layers}

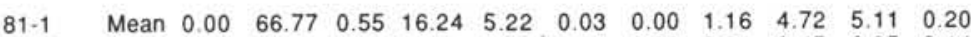
$\begin{array}{lllllllllllllll}\mathrm{n}=10 & \text { SD } & 0.00 & 1.18 & 0.13 & 1.28 & 0.68 & 0.06 & 0.00 & 0.31 & 0.45 & 0.95 & 0.14\end{array}$

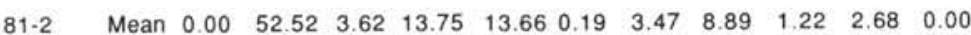
$\begin{array}{lllllllllllll}\mathrm{n}=7 & \mathrm{SD} & 0.00 & 1.19 & 0.15 & 0.70 & 0.73 & 0.03 & 0.27 & 0.48 & 0.33 & 0.29 & 0.00\end{array}$

$82 \quad \begin{array}{llllllllllll}\text { 8. } & 0.00 & 52.33 & 3.56 & 13.52 & 13.88 & 0.10 & 3.80 & 8.94 & 1.15 & 2.71 & 0.02\end{array}$ $\begin{array}{lllllllllllll}n=17 & S D & 0.00 & 0.33 & 0.10 & 0.17 & 0.28 & 0.09 & 0.12 & 0.26 & 0.09 & 0.17 & 0.06\end{array}$

\section{Hole $737 \mathrm{~A}$, dispersed ash}

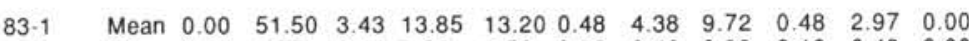
$\begin{array}{llllllllllllll}\mathrm{n}=7 & \mathrm{SD} & 0.00 & 2.75 & 0.44 & 1.02 & 1.50 & 0.16 & 0.46 & 0.99 & 0.10 & 0.43 & 0.00\end{array}$

83-2 $\quad \begin{array}{llllllllllll}\text { 8. } & 0.00 & 50.30 & 3.64 & 14.94 & 12.86 & 0.51 & 3.71 & 9.23 & 1.23 & 3.58 & 0.01\end{array}$ $\begin{array}{lllllllllllll}n=9 & \text { SD } & 0.00 & 0.41 & 0.25 & 0.45 & 0.74 & 0.21 & 0.59 & 0.84 & 0.44 & 0.35 & 0.02\end{array}$

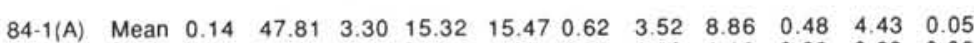
$\begin{array}{lllllllllllll}\mathrm{n}=4 & \mathrm{SD} & 0.28 & 1.81 & 0.59 & 0.86 & 1.53 & 0.06 & 1.20 & 1.16 & 0.09 & 0.68 & 0.06\end{array}$ 
Table 4 (continued).

\begin{tabular}{|c|c|c|c|c|c|c|c|c|c|c|c|c|}
\hline $\begin{array}{l}\text { AWl- } \\
\text { number }\end{array}$ & & $\begin{array}{c}\mathrm{P}_{2} \mathrm{O}_{5} \\
(\%)\end{array}$ & $\begin{array}{c}\mathrm{SiO}_{2} \\
(\%)\end{array}$ & $\begin{array}{l}\mathrm{TiO}_{2} \\
(\%)\end{array}$ & $\begin{array}{c}\mathrm{Al}_{2} \mathrm{O}_{3} \\
(\%)\end{array}$ & $\begin{array}{c}\mathrm{FeO} \\
(\%)\end{array}$ & $\begin{array}{c}\mathrm{MnO} \\
(\%)\end{array}$ & $\begin{array}{c}\mathrm{MgO} \\
(\%)\end{array}$ & $\begin{array}{l}\mathrm{CaO} \quad \mathrm{K} \\
(\%)\end{array}$ & $\begin{array}{c}\mathrm{K}_{2} \mathrm{O} \\
(\%)\end{array}$ & $\begin{array}{c}\mathrm{Na}_{2} \mathrm{O} \\
(\%)\end{array}$ & $\begin{array}{l}\mathrm{Cl} \\
(\%)\end{array}$ \\
\hline $\begin{array}{l}84-1(B) \\
n=2\end{array}$ & Mean & 0.00 & 61.84 & 0.83 & 17.01 & 5.46 & 0.00 & 0.00 & 1.81 & 7.46 & 5.56 & 0.04 \\
\hline $\begin{array}{l}84-2 \\
n=10\end{array}$ & $\begin{array}{l}\text { Mean } \\
\text { SD }\end{array}$ & $\begin{array}{l}0.12 \\
0.26\end{array}$ & $\begin{array}{l}48.78 \\
2.02\end{array}$ & $\begin{array}{l}3.26 \\
0.53\end{array}$ & $\begin{array}{l}15.46 \\
0.88\end{array}$ & $\begin{array}{l}12.61 \\
1.58\end{array}$ & $\begin{array}{l}0.19 \\
0.16\end{array}$ & $\begin{array}{l}4.71 \\
1.21\end{array}$ & $\begin{array}{l}10.71 \\
0.95\end{array}$ & $\begin{array}{l}1.01 \\
0.40\end{array}$ & $\begin{array}{l}3.15 \\
0.39\end{array}$ & $\begin{array}{l}0.01 \\
0.03\end{array}$ \\
\hline \multicolumn{13}{|c|}{ Hole $745 \mathrm{~B}$, discrete ash layers } \\
\hline $\begin{array}{l}85 \\
n=14\end{array}$ & $\begin{array}{l}\text { Mean } \\
\text { SD }\end{array}$ & $\begin{array}{l}0.00 \\
0.00\end{array}$ & $\begin{array}{l}62.64 \\
1.25\end{array}$ & $\begin{array}{l}0.79 \\
0.19\end{array}$ & $\begin{array}{l}16.99 \\
0.61\end{array}$ & $\begin{array}{l}5.57 \\
0.24\end{array}$ & $\begin{array}{l}0.08 \\
0.10\end{array}$ & $\begin{array}{l}0.00 \\
0.00\end{array}$ & $\begin{array}{l}1.75 \\
0.31\end{array}$ & $\begin{array}{l}6.36 \\
0.35\end{array}$ & $\begin{array}{l}5.75 \\
0.27\end{array}$ & $\begin{array}{l}0.06 \\
0.05\end{array}$ \\
\hline $\begin{array}{l}86-1 \\
n=11\end{array}$ & $\begin{array}{l}\text { Mean } \\
\text { SD }\end{array}$ & $\begin{array}{l}0.00 \\
0.00\end{array}$ & $\begin{array}{l}64.48 \\
0.60\end{array}$ & $\begin{array}{l}0.64 \\
0.04\end{array}$ & $\begin{array}{l}15.85 \\
0.69\end{array}$ & $\begin{array}{l}5.30 \\
0.44\end{array}$ & $\begin{array}{l}0.11 \\
0.11\end{array}$ & $\begin{array}{l}0.00 \\
0.00\end{array}$ & $\begin{array}{l}1.11 \\
0.15\end{array}$ & $\begin{array}{l}6.01 \\
0.41\end{array}$ & $\begin{array}{l}6.35 \\
0.43\end{array}$ & $\begin{array}{l}0.15 \\
0.04\end{array}$ \\
\hline Hole 745 & $5 \mathrm{~B}$ anc & 746 & A, disp & persed & ash & & & & & & & \\
\hline $\begin{array}{l}111-1 \\
n=4\end{array}$ & Mean & 0.11 & 46.42 & 3.07 & 15.70 & 12.04 & 0.27 & 5.57 & 12.09 & 1.29 & 3.42 & 0.03 \\
\hline $\begin{array}{l}111-2(A) \\
n=4\end{array}$ & Mean & 0.34 & 45.99 & 2.87 & 17.19 & 10.91 & 0.25 & 4.82 & 11.08 & 1.85 & 4.61 & 0.10 \\
\hline $111-2(\mathrm{~B})$ & & 0.00 & 54.61 & 1.52 & 18.44 & 8.89 & 0.22 & 1.12 & 4.81 & 3.57 & 6.67 & 0.14 \\
\hline $\begin{array}{l}111-3(A) \\
\mathrm{n}=2\end{array}$ & Mean & 0.27 & 46.54 & 3.22 & 16.05 & 10.68 & 0.27 & 4.81 & 11.90 & 2.00 & 4.18 & 0.11 \\
\hline $111-3$ & & 1.19 & 50.30 & 2.67 & 16.24 & 10.90 & 0.23 & 2.74 & 6.96 & 2.49 & 6.05 & 0.22 \\
\hline $\begin{array}{l}111-3 \\
111-3\end{array}$ & & $\begin{array}{l}0.19 \\
0.00\end{array}$ & $\begin{array}{l}57.95 \\
63.34\end{array}$ & $\begin{array}{l}1.36 \\
0.54\end{array}$ & $\begin{array}{l}19.15 \\
13.70\end{array}$ & $\begin{array}{l}7.08 \\
8.12\end{array}$ & $\begin{array}{l}0.15 \\
0.24\end{array}$ & $\begin{array}{l}0.00 \\
0.00\end{array}$ & $\begin{array}{l}3.59 \\
0.99\end{array}$ & $\begin{array}{l}4.69 \\
4.82\end{array}$ & $\begin{array}{l}5.74 \\
8.03\end{array}$ & $\begin{array}{l}0.11 \\
0.22\end{array}$ \\
\hline $\begin{array}{l}111-4 \\
n=2\end{array}$ & Mean & 0.00 & 74.29 & 0.21 & 13.23 & 2.05 & 0.00 & 0.00 & 0.51 & 5.08 & 4.36 & 0.29 \\
\hline $\begin{array}{l}112-1(A) \\
n=3\end{array}$ & Mean & 0.00 & 48.68 & 1.76 & 17.12 & 11.14 & 0.23 & 6.05 & 10.25 & 0.83 & 3.91 & 0.05 \\
\hline $\begin{array}{l}112-1(B) \\
n=2\end{array}$ & Mean & 0.00 & 61.07 & 0.66 & 12.82 & 10.31 & 0.38 & 0.00 & 1.15 & 4.61 & 8.59 & 0.35 \\
\hline $\begin{array}{l}112-1(C) \\
n=2\end{array}$ & Mean & 0.00 & 74.47 & 0.19 & 11.98 & 2.83 & 0.10 & 0.00 & 0.25 & 4.98 & 4.88 & 0.33 \\
\hline $\begin{array}{l}112-2(A) \\
n=2\end{array}$ & Mean & 0.00 & 45.83 & 3.10 & 16.12 & 11.55 & 0.30 & 5.90 & 12.81 & 1.21 & 3.16 & 0.04 \\
\hline $112-2(\mathrm{~B})$ & & 0.00 & 69.74 & 0.29 & 14.06 & 3.76 & 0.16 & 0.00 & 0.56 & 5.57 & 5.51 & 0.36 \\
\hline $\begin{array}{l}112-2(C) \\
n=3\end{array}$ & Mean & 0.00 & 75.25 & 0.14 & 11.57 & 2.79 & 0.00 & 0.00 & 0.24 & 4.79 & 4.78 & 0.45 \\
\hline $\begin{array}{l}112-3(A) \\
n=5\end{array}$ & $\begin{array}{l}\text { Mean } \\
\text { SD }\end{array}$ & $\begin{array}{l}0.43 \\
0.10\end{array}$ & $\begin{array}{r}46.62 \\
0.26\end{array}$ & $\begin{array}{l}3.32 \\
0.07\end{array}$ & $\begin{array}{r}16.11 \\
0.20\end{array}$ & $\begin{array}{r}11.81 \\
0.09\end{array}$ & $\begin{array}{l}0.25 \\
0.06\end{array}$ & $\begin{array}{l}5.03 \\
0.07\end{array}$ & $\begin{array}{r}10.85 \\
0.03\end{array}$ & $\begin{array}{l}1.39 \\
0.05\end{array}$ & $\begin{array}{l}4.19 \\
0.21\end{array}$ & $\begin{array}{l}0.01 \\
0.03\end{array}$ \\
\hline $\begin{array}{l}112-3(B) \\
n=2\end{array}$ & Mean & 0.00 & 71.54 & 0.34 & 9.61 & 6.34 & 0.32 & 0.00 & 0.27 & 4.51 & 6.30 & 0.80 \\
\hline $113-1$ & & 0.53 & 45.20 & 4.01 & 15.78 & 13.27 & 0.27 & 3.65 & 10.53 & 1.99 & 4.69 & 0.08 \\
\hline $113-2(A)$ & & 0.17 & 50.37 & 3.79 & 14.53 & 12.96 & 0.29 & 3.65 & 10.53 & 1.29 & 3.71 & 0.00 \\
\hline $\begin{array}{l}113-2(B) \\
n=5\end{array}$ & $\begin{array}{l}\text { Mean } \\
\text { SD }\end{array}$ & $\begin{array}{l}0.00 \\
0.00\end{array}$ & $\begin{array}{r}55.54 \\
0.78\end{array}$ & $\begin{array}{l}1.21 \\
0.30\end{array}$ & $\begin{array}{r}15.44 \\
1.04\end{array}$ & $\begin{array}{r}11.58 \\
1.25\end{array}$ & $\begin{array}{l}0.26 \\
0.03\end{array}$ & $\begin{array}{l}3.81 \\
0.43\end{array}$ & $\begin{array}{l}8.78 \\
0.30\end{array}$ & $\begin{array}{l}0.50 \\
0.11\end{array}$ & $\begin{array}{l}2.75 \\
0.36\end{array}$ & $\begin{array}{l}0.12 \\
0.06\end{array}$ \\
\hline $\begin{array}{l}113-2(C) \\
n=3\end{array}$ & Mean & 0.01 & 60.48 & 1.02 & 16.60 & 7.45 & 0.18 & 1.13 & 3.86 & 4.05 & 5.08 & 0.13 \\
\hline $\begin{array}{l}113-4(A) \\
n=3\end{array}$ & Mean & 0.00 & 55.27 & 1.33 & 16.19 & 10.71 & 0.19 & 3.68 & 8.50 & 0.65 & 3.39 & 0.09 \\
\hline $\begin{array}{l}113-4(B) \\
n=2\end{array}$ & Mean & 0.00 & 66.30 & 0.31 & 16.39 & 3.63 & 0.00 & 0.00 & 0.64 & 5.59 & 6.83 & 0.34 \\
\hline $\begin{array}{l}113-5 \\
n=3\end{array}$ & Mean & 0.00 & 64.73 & 0.42 & 16.75 & 4.39 & 0.12 & 0.00 & 1.26 & 5.76 & 6.41 & 0.16 \\
\hline $\begin{array}{l}114-1 \\
n=4\end{array}$ & Mean & 0.00 & 66.97 & 0.58 & 15.42 & 3.62 & 0.27 & 0.00 & 0.56 & 5.98 & 6.35 & 0.26 \\
\hline $114-1$ & & 0.00 & 74.47 & 0.22 & 12.42 & 2.85 & 0.00 & 0.00 & 0.29 & 4.66 & 4.82 & 0.27 \\
\hline $\begin{array}{l}114-2(A) \\
n=5\end{array}$ & Mean & 0.78 & 45.74 & 3.48 & 17.41 & 11.75 & 0.28 & 3.67 & 9.32 & 2.38 & 4.92 & 0.14 \\
\hline $\begin{array}{l}114-2(B) \\
n=2\end{array}$ & Mean & 0.00 & 64.75 & 0.54 & 16.39 & 4.11 & 0.27 & 0.00 & 0.84 & 6.32 & 6.51 & 0.30 \\
\hline $\begin{array}{l}114-5 \\
n=4\end{array}$ & Mean & 0.47 & 49.39 & 2.64 & 15.77 & 12.83 & 0.24 & 3.79 & 8.38 & 1.92 & 4.50 & 0.07 \\
\hline $\begin{array}{l}115-2 \\
n=8\end{array}$ & $\begin{array}{l}\text { Mean } \\
\text { SD }\end{array}$ & $\begin{array}{l}0.00 \\
0.00\end{array}$ & $\begin{array}{r}64.13 \\
0.50\end{array}$ & $\begin{array}{l}0.49 \\
0.13\end{array}$ & $\begin{array}{r}15.68 \\
0.53\end{array}$ & $\begin{array}{l}5.72 \\
0.28\end{array}$ & $\begin{array}{l}0.27 \\
0.06\end{array}$ & $\begin{array}{l}0.00 \\
0.00\end{array}$ & $\begin{array}{l}1.03 \\
0.29\end{array}$ & $\begin{array}{l}5.26 \\
0.40\end{array}$ & $\begin{array}{l}7.24 \\
0.63\end{array}$ & $\begin{array}{l}0.18 \\
0.06\end{array}$ \\
\hline $\begin{array}{l}115-3(A) \\
n=8\end{array}$ & $\begin{array}{l}\text { Mean } \\
\text { SD }\end{array}$ & $\begin{array}{l}0.52 \\
0.41\end{array}$ & $\begin{array}{r}45.80 \\
1.34\end{array}$ & $\begin{array}{l}3.52 \\
0.33\end{array}$ & $\begin{array}{r}16.28 \\
1.81\end{array}$ & $\begin{array}{r}12.10 \\
1.80\end{array}$ & $\begin{array}{l}0.24 \\
0.04\end{array}$ & $\begin{array}{l}4.77 \\
1.43\end{array}$ & $\begin{array}{r}10.63 \\
1.75\end{array}$ & $\begin{array}{l}1.76 \\
0.70\end{array}$ & $\begin{array}{l}4.29 \\
0.71\end{array}$ & $\begin{array}{l}0.06 \\
0.05\end{array}$ \\
\hline
\end{tabular}


Table 4 (continued).

\begin{tabular}{|c|c|c|c|c|c|c|c|c|c|c|c|c|}
\hline $\begin{array}{l}\text { AWI- } \\
\text { number }\end{array}$ & & $\begin{array}{c}\mathrm{P}_{2} \mathrm{O}_{5} \\
(\%)\end{array}$ & $\begin{array}{c}\mathrm{SiO}_{2} \\
(\%)\end{array}$ & $\begin{array}{l}\mathrm{TiO}_{2} \\
(\%)\end{array}$ & $\begin{array}{c}\mathrm{Al}_{2} \mathrm{O}_{3} \\
(\%)\end{array}$ & $\begin{array}{l}\mathrm{FeO} \\
(\%)\end{array}$ & $\begin{array}{c}\mathrm{MnO} \\
(\%)\end{array}$ & $\begin{array}{c}\mathrm{MgO} \\
(\%)\end{array}$ & $\begin{array}{l}\mathrm{CaO} \\
(\%)\end{array}$ & $\begin{array}{l}\mathrm{K}_{2} \mathrm{O} \\
(\%)\end{array}$ & $\begin{array}{c}\mathrm{Na}_{2} \mathrm{O} \\
(\%)\end{array}$ & $\begin{array}{c}\mathrm{Cl} \\
(\%)\end{array}$ \\
\hline $\begin{array}{l}115-3(B) \\
n=3\end{array}$ & Mean & 0.00 & 57.46 & 1.37 & 15.59 & 10.21 & 0.22 & 2.71 & 7.88 & 0.79 & 3.67 & 0.11 \\
\hline $\begin{array}{l}115-5 \\
n=5\end{array}$ & $\begin{array}{l}\text { Mean } \\
\text { SD }\end{array}$ & $\begin{array}{l}0.75 \\
0.39\end{array}$ & $\begin{array}{r}45.65 \\
1.97\end{array}$ & $\begin{array}{l}3.63 \\
0.28\end{array}$ & $\begin{array}{r}15.77 \\
1.01\end{array}$ & $\begin{array}{r}12.27 \\
0.87\end{array}$ & $\begin{array}{l}0.21 \\
0.02\end{array}$ & $\begin{array}{l}4.33 \\
0.32\end{array}$ & $\begin{array}{r}10.62 \\
1.03\end{array}$ & $\begin{array}{l}1.99 \\
0.51\end{array}$ & $\begin{array}{l}4.70 \\
0.86\end{array}$ & $\begin{array}{l}0.11 \\
0.07\end{array}$ \\
\hline $\begin{array}{l}117-1 \\
n=5\end{array}$ & $\begin{array}{l}\text { Mean } \\
\text { SD }\end{array}$ & $\begin{array}{l}0.83 \\
0.12\end{array}$ & $\begin{array}{r}43.29 \\
0.81\end{array}$ & $\begin{array}{l}4.08 \\
0.21\end{array}$ & $\begin{array}{r}16.52 \\
0.85\end{array}$ & $\begin{array}{r}13.07 \\
0.81\end{array}$ & $\begin{array}{l}0.26 \\
0.07\end{array}$ & $\begin{array}{l}4.08 \\
0.37\end{array}$ & $\begin{array}{r}11.50 \\
0.34\end{array}$ & $\begin{array}{l}2.05 \\
0.15\end{array}$ & $\begin{array}{l}4.22 \\
0.47\end{array}$ & $\begin{array}{l}0.09 \\
0.06\end{array}$ \\
\hline $\begin{array}{l}117-2 \\
n=3\end{array}$ & Mean & 0.00 & 57.34 & 1.44 & 14.53 & 11.36 & 0.27 & 3.32 & 7.76 & 0.76 & 3.14 & 0.08 \\
\hline $\begin{array}{l}1.17-3 \\
n=11\end{array}$ & $\begin{array}{l}\text { Mean } \\
\text { SD }\end{array}$ & $\begin{array}{l}0.85 \\
0.32\end{array}$ & $\begin{array}{r}45.15 \\
1.90\end{array}$ & $\begin{array}{l}3.27 \\
0.65\end{array}$ & $\begin{array}{r}16.82 \\
0.84\end{array}$ & $\begin{array}{r}11.11 \\
0.36\end{array}$ & $\begin{array}{l}0.27 \\
0.06\end{array}$ & $\begin{array}{l}4.05 \\
0.61\end{array}$ & $\begin{array}{r}11.05 \\
1.66\end{array}$ & $\begin{array}{l}2.32 \\
0.23\end{array}$ & $\begin{array}{l}4.98 \\
0.47\end{array}$ & $\begin{array}{l}0.17 \\
0.04\end{array}$ \\
\hline $\begin{array}{l}117-4(A) \\
n=2\end{array}$ & Mean & 0.82 & 46.98 & 3.65 & 16.41 & 11.53 & 0.34 & 3.35 & 9.15 & 2.51 & 5.20 & 0.09 \\
\hline $\begin{array}{l}117-4(B) \\
n=3\end{array}$ & Mean & 0.00 & 63.75 & 0.44 & 17.12 & 4.60 & 0.13 & 0.00 & 1.12 & 5.89 & 6.71 & 0.24 \\
\hline $\begin{array}{l}117-5(A) \\
n=6\end{array}$ & $\begin{array}{l}\text { Mean } \\
\text { SD }\end{array}$ & $\begin{array}{l}0.61 \\
0.24\end{array}$ & $\begin{array}{r}48.46 \\
1.25\end{array}$ & $\begin{array}{l}2.76 \\
0.21\end{array}$ & $\begin{array}{r}18.30 \\
0.59\end{array}$ & $\begin{array}{r}10.04 \\
0.36\end{array}$ & $\begin{array}{l}0.20 \\
0.10\end{array}$ & $\begin{array}{l}2.65 \\
0.50\end{array}$ & $\begin{array}{l}8.56 \\
1.31\end{array}$ & $\begin{array}{l}2.44 \\
0.64\end{array}$ & $\begin{array}{l}5.80 \\
0.89\end{array}$ & $\begin{array}{l}0.19 \\
0.07\end{array}$ \\
\hline $117-5(B)$ & & 0.00 & 67.42 & 0.63 & 16.07 & 3.36 & 0.22 & 0.00 & 0.50 & 6.38 & 5.28 & 0.15 \\
\hline $\begin{array}{l}118-1 \\
n=3\end{array}$ & Mean & 0.41 & 45.74 & 3.84 & 16.81 & 11.92 & 0.24 & 4.41 & 10.53 & 1.94 & 4.08 & 0.07 \\
\hline $\begin{array}{l}118-3 \\
n=3\end{array}$ & Mean & 0.00 & 48.83 & 2.08 & 16.51 & 11.29 & 0.17 & 5.77 & 10.46 & 0.90 & 3.88 & 0.05 \\
\hline $\begin{array}{l}118-4 \\
n=3\end{array}$ & Mean & 0.52 & 45.54 & 3.06 & 17.13 & 11.93 & 0.31 & 3.98 & 10.65 & 2.14 & 4.64 & 0.10 \\
\hline $\begin{array}{l}118-5 \\
n=2\end{array}$ & Mean & 0.23 & 49.86 & 1.90 & 18.90 & 9.56 & 0.21 & 3.94 & 8.62 & 1.99 & 4.73 & 0.08 \\
\hline $\begin{array}{l}119-2 \\
n=7\end{array}$ & $\begin{array}{l}\text { Mean } \\
\text { SD }\end{array}$ & $\begin{array}{l}0.00 \\
0.00\end{array}$ & $\begin{array}{r}47.40 \\
0.49\end{array}$ & $\begin{array}{l}2.21 \\
0.14\end{array}$ & $\begin{array}{r}16.66 \\
0.14\end{array}$ & $\begin{array}{r}11.55 \\
0.33\end{array}$ & $\begin{array}{l}0.21 \\
0.05\end{array}$ & $\begin{array}{l}6.44 \\
0.10\end{array}$ & $\begin{array}{r}10.32 \\
0.13\end{array}$ & $\begin{array}{l}0.96 \\
0.06\end{array}$ & $\begin{array}{l}4.17 \\
0.23\end{array}$ & $\begin{array}{l}0.03 \\
0.04\end{array}$ \\
\hline $\begin{array}{l}119-3(\mathrm{~A}) \\
119-3(\mathrm{~B}) \\
\mathrm{n}=3 \\
119-3(\mathrm{C})\end{array}$ & Mean & $\begin{array}{l}1.02 \\
0.00\end{array}$ & $\begin{array}{l}46.70 \\
67.79\end{array}$ & $\begin{array}{l}3.89 \\
0.51\end{array}$ & $\begin{array}{l}14.70 \\
14.56\end{array}$ & $\begin{array}{l}14.88 \\
4.90\end{array}$ & $\begin{array}{l}0.25 \\
0.24 \\
0.00\end{array}$ & $\begin{array}{l}4.00 \\
0.00\end{array}$ & $\begin{array}{l}9.19 \\
1.45 \\
\\
3.98\end{array}$ & $\begin{array}{l}1.28 \\
5.26 \\
2.20\end{array}$ & $\begin{array}{l}4.10 \\
5.14 \\
\\
8.33\end{array}$ & $\begin{array}{l}0.00 \\
0.15 \\
0.00\end{array}$ \\
\hline $\begin{array}{l}116-1 \\
n=2\end{array}$ & Mean & 0.81 & 47.71 & 3.65 & 15.43 & 13.63 & 0.29 & 3.65 & 8.37 & 1.88 & 4.44 & 0.09 \\
\hline $116-2$ & & 0.00 & 64.40 & 0.77 & 16.14 & 5.15 & 0.17 & 0.00 & 1.46 & 6.06 & 5.84 & 0.00 \\
\hline $\begin{array}{l}116 \cdot 3(\mathrm{~A}) \\
116 \cdot 3(\mathrm{~B}) \\
\mathrm{n}=3\end{array}$ & Mean & $\begin{array}{l}0.23 \\
0.00\end{array}$ & $\begin{array}{l}45.16 \\
63.12\end{array}$ & $\begin{array}{l}3.24 \\
0.71\end{array}$ & $\begin{array}{l}17.07 \\
16.74\end{array}$ & $\begin{array}{l}10.94 \\
5.42\end{array}$ & $\begin{array}{l}0.25 \\
0.22\end{array}$ & $\begin{array}{l}5.42 \\
0.00\end{array}$ & $\begin{array}{l}11.98 \\
1.59\end{array}$ & $\begin{array}{l}1.60 \\
5.93\end{array}$ & $\begin{array}{l}4.12 \\
6.16\end{array}$ & $\begin{array}{l}0.00 \\
0.11\end{array}$ \\
\hline $\begin{array}{l}116 \cdot 4(A) \\
n=2\end{array}$ & Mean & 0.36 & 45.70 & 2.86 & 17.21 & 12.16 & 0.19 & 6.60 & 10.39 & 0.94 & 3.62 & 0.00 \\
\hline $\begin{array}{l}116-4(B) \\
n=6\end{array}$ & $\begin{array}{l}\text { Mean } \\
\text { SD }\end{array}$ & $\begin{array}{l}0.00 \\
0.00\end{array}$ & $\begin{array}{l}64.33 \\
2.29\end{array}$ & $\begin{array}{l}0.80 \\
0.30\end{array}$ & $\begin{array}{l}15.88 \\
1.25\end{array}$ & $\begin{array}{l}5.41 \\
0.22\end{array}$ & $\begin{array}{l}0.26 \\
0.08\end{array}$ & $\begin{array}{l}0.00 \\
0.00\end{array}$ & $\begin{array}{l}1.52 \\
0.60\end{array}$ & $\begin{array}{l}5.85 \\
0.47\end{array}$ & $\begin{array}{l}5.86 \\
0.44\end{array}$ & $\begin{array}{l}0.08 \\
0.08\end{array}$ \\
\hline $\begin{array}{l}116.5 \\
116-5\end{array}$ & & $\begin{array}{l}0.00 \\
0.00\end{array}$ & $\begin{array}{l}55.44 \\
61.74\end{array}$ & $\begin{array}{l}1.31 \\
1.17\end{array}$ & $\begin{array}{l}17.02 \\
17.37\end{array}$ & $\begin{array}{l}9.05 \\
5.78\end{array}$ & $\begin{array}{l}0.18 \\
0.27\end{array}$ & $\begin{array}{l}3.39 \\
0.00\end{array}$ & $\begin{array}{l}8.15 \\
2.21\end{array}$ & $\begin{array}{l}1.04 \\
6.14\end{array}$ & $\begin{array}{l}4.22 \\
5.31\end{array}$ & $\begin{array}{l}0.20 \\
0.00\end{array}$ \\
\hline $\begin{array}{l}146-1 \\
n=11\end{array}$ & $\begin{array}{l}\text { Mean } \\
\text { SD }\end{array}$ & $\begin{array}{l}0.46 \\
0.23\end{array}$ & $\begin{array}{l}47.38 \\
2.70\end{array}$ & $\begin{array}{l}3.01 \\
0.61\end{array}$ & $\begin{array}{l}17.21 \\
0.82\end{array}$ & $\begin{array}{l}10.60 \\
1.06\end{array}$ & $\begin{array}{l}0.24 \\
0.05\end{array}$ & $\begin{array}{l}3.97 \\
1.06\end{array}$ & $\begin{array}{l}9.93 \\
2.01\end{array}$ & $\begin{array}{l}2.15 \\
0.75\end{array}$ & $\begin{array}{l}4.94 \\
0.74\end{array}$ & $\begin{array}{l}0.10 \\
0.08\end{array}$ \\
\hline $\begin{array}{l}146-2 \\
n=4\end{array}$ & $\begin{array}{l}\text { Mean } \\
\text { SD }\end{array}$ & $\begin{array}{l}0.00 \\
0.00\end{array}$ & $\begin{array}{l}67.73 \\
0.30\end{array}$ & $\begin{array}{l}0.43 \\
0.09\end{array}$ & $\begin{array}{l}14.84 \\
0.12\end{array}$ & $\begin{array}{l}4.46 \\
0.10\end{array}$ & $\begin{array}{l}0.18 \\
0.04\end{array}$ & $\begin{array}{l}0.00 \\
0.00\end{array}$ & $\begin{array}{l}0.80 \\
0.04\end{array}$ & $\begin{array}{l}5.45 \\
0.07\end{array}$ & $\begin{array}{l}5.92 \\
0.24\end{array}$ & $\begin{array}{l}0.19 \\
0.02\end{array}$ \\
\hline $\begin{array}{l}146 \cdot 3(A) \\
n=2\end{array}$ & & 0.29 & 47.07 & 2.99 & 16.19 & 12.90 & 0.31 & 5.45 & 10.18 & 0.97 & 3.53 & 0.03 \\
\hline $\begin{array}{l}146-3(B) \\
n=10\end{array}$ & $\begin{array}{l}\text { Mean } \\
\text { SD }\end{array}$ & $\begin{array}{l}0.00 \\
0.00\end{array}$ & $\begin{array}{l}61.89 \\
0.24\end{array}$ & $\begin{array}{l}0.68 \\
0.04\end{array}$ & $\begin{array}{l}12.87 \\
1.72\end{array}$ & $\begin{array}{l}9.97 \\
1.26\end{array}$ & $\begin{array}{l}0.33 \\
0.04\end{array}$ & $\begin{array}{l}0.00 \\
0.00\end{array}$ & $\begin{array}{l}1.40 \\
0.37\end{array}$ & $\begin{array}{l}4.73 \\
0.31\end{array}$ & $\begin{array}{l}7.85 \\
1.06\end{array}$ & $\begin{array}{l}0.25 \\
0.08\end{array}$ \\
\hline $\begin{array}{l}147-2(A) \\
n=8\end{array}$ & $\begin{array}{l}\text { Mean } \\
\text { SD }\end{array}$ & $\begin{array}{l}0.11 \\
0.24\end{array}$ & $\begin{array}{l}49.72 \\
2.42\end{array}$ & $\begin{array}{l}2.87 \\
0.54\end{array}$ & $\begin{array}{l}15.96 \\
0.38\end{array}$ & $\begin{array}{l}12.35 \\
0.57\end{array}$ & $\begin{array}{l}0.28 \\
0.04\end{array}$ & $\begin{array}{l}3.74 \\
0.65\end{array}$ & $\begin{array}{l}9.09 \\
0.88\end{array}$ & $\begin{array}{l}1.70 \\
0.29\end{array}$ & $\begin{array}{l}4.12 \\
0.23\end{array}$ & $\begin{array}{l}0.04 \\
0.05\end{array}$ \\
\hline $147-2(B)$ & & 0.00 & 75.67 & 0.20 & 11.32 & 3.10 & 0.00 & 0.00 & 0.27 & 4.80 & 4.44 & 0.21 \\
\hline
\end{tabular}

a " $n "$ = number of individual glass shards analysed from a single ash layer; "SD" = standard deviation. 


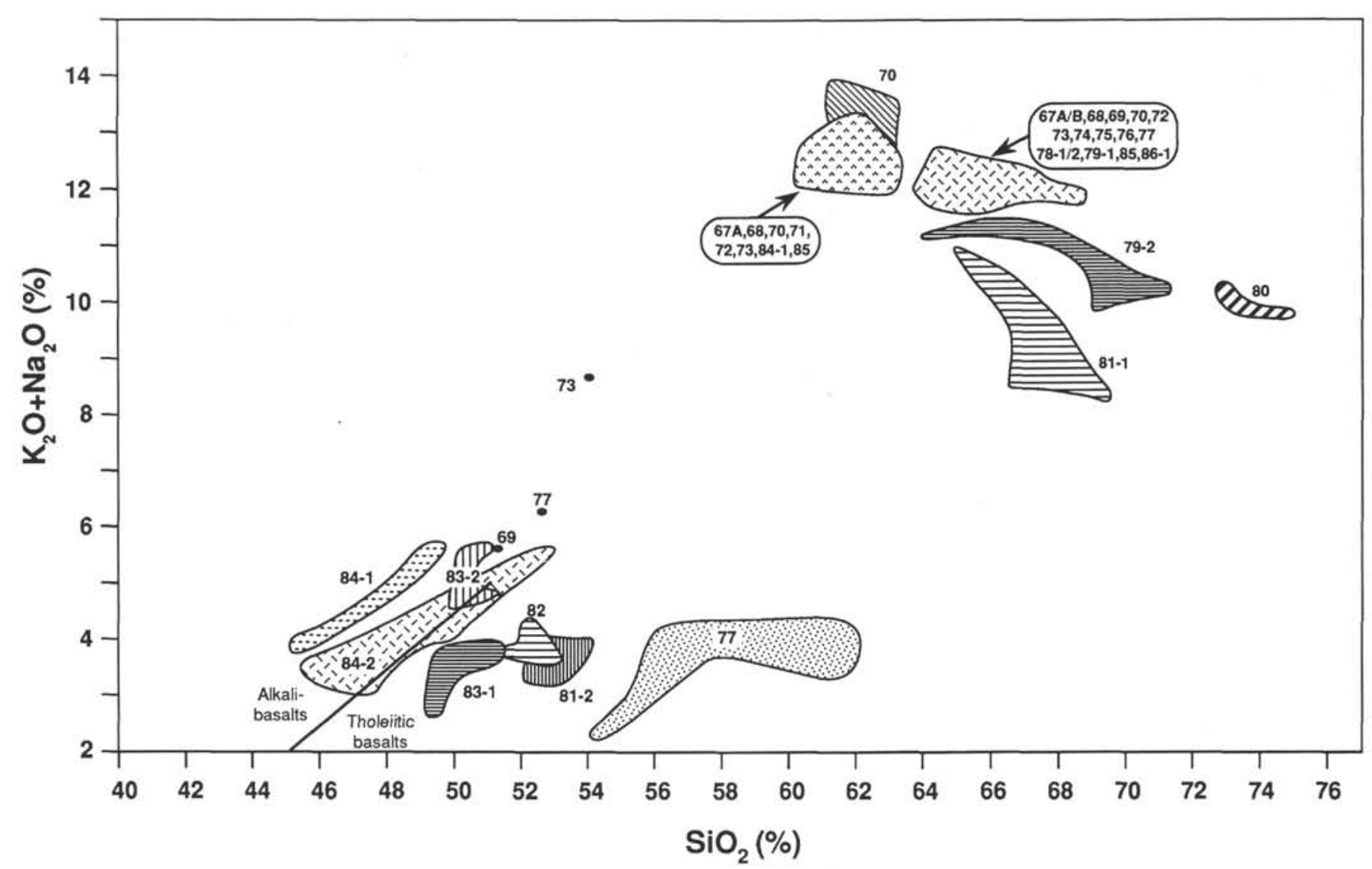

Figure 5. Total alkali vs. silica (TAS) diagram for the discrete tephra layers from Sites $736,737,745$, and dispersed ash from Site 737 . The alkali to tholeiitic basalt separation line is after Macdonald and Katsura (1964).

total the same two petrographic groups as defined for discrete tephra layers at Sites 736,737 , and 745 , that is the basaltic and the trachytic to (alkali-) rhyolitic groups. In contrast to the northern Kerguelen Plateau sites, both groups span the interval from late Miocene to Quaternary virtually without any systematic variation of chemistry with time. The overall chemical similarity with the magmatic rocks from the Kerguelen Islands argues for a provenance from that area, probably as subaerially transported material. Since volcanic activity of Heard Island can be regarded as contemporaneously and very similar in geochemical evolution to the Kerguelen Islands it represents an important source especially for the basaltic and basanitic tephras. On the other hand, the seismic reflection profiles at the sites indicate a remarkable degree of redistribution of sediment by bottom currents. The possible influence of other sources for the tephra, such as volcanic structures on the Kerguelen Plateau now eroded or subsided (e.g., Fröhlich and Wicquart, 1989) has to be considered.

Transport of additional tephra to the site of deposition by ice rafting is also possible, since the sites are situated in an area characterized by a high frequency of drifting icebergs (Barron, Larsen, et al., 1989). Indeed, ice-rafted material has been identified throughout the sedimentary sequence recovered at Sites 745 and 746. However, all of the detrital ice-rafted material is considered to be derived from icebergs originating from the Antarctic continent (Ehrmann et al., this volume). On the other hand, sea-ice rafting also has been an important transport process for the volcanic ashes, because the sites are situated far south of the mean winter sea-ice edge (Fig. 1). Examples for such transport are the "exotic" calc-alkaline tephras occurring, for which the South Sandwich island arc as source area is suggested.

\section{ACKNOWLEDGMENTS}

We thank Heike Ostermann (AWI) and Erika Lutz (Freiburg University) for technical help and assistance during sample processing and microprobe measurements. Financial support by the Deutsche Forschungsgemeinschaft (Grants Fu 119/14 and $\mathrm{Hu}$ $378 / 2$ ) is acknowledged. Michael J. Hambrey improved the English text of an earlier version of the manuscript. This is AWI publication No. 329.

\section{REFERENCES}

Baker, P. E., 1978. The South Sandwich Islands: III. Petrology of the volcanic rocks. Sci. Rept. Br. Antarct. Surv., 93.

Barron, J., Larsen, B., et al., 1989. Proc. ODP, Init. Repts., 119: College Station, TX (Ocean Drilling Program).

Chevallier, L., Nougier, J., and Cantagrel, J. M., 1983. Volcanology of Possession Island, Crozet Archipelago (TAAF). In Oliver, R. L., James, P. R., and Jago, J. B. (Eds.), Antarctic Earth Science: Cambridge (Cambridge Univ. Press), 652-658.

Clarke, I., McDougall, I., and Whitford, D. J., 1983. Volcanic evolution of Heard and McDonald Islands, southern Indian Ocean. In Oliver, R. L., James, P. R., and Jago, J. B. (Eds.), Antarctic Earth Science: Cambridge (Cambridge Univ. Press), 631-635.

Dietrich, G., and Ulrich, J., 1968. Atlas zur Ozeanographie: Mannheim (Bibliographisches Institut).

Fisher, R. L., Jantsch, M. Z., and Comer, R. L., 1982. General Bathymetric Chart of the Oceans (GEBCO), Scale 1:10,000,000: Ottawa (Canadian Hydrographic Service), 5.9.

Fröhlich, F., and Wicquart, E., 1989. Upper Cretaceous and Paleogene sediments from the northern Kerguelen Plateau. Geo-Mar. Lett., 9: 127-133.

Giret, A., 1983. Le plutonisme océanique intraplaque. Exemple des Iles Kerguelen. Com. Nat. Fr. Rech. Antarct., 54. 
Giret, A., and Lameyre, J., 1983. A study of Kerguelen plutonism: petrology, geochronology and geological implications. In Oliver, R. L., James, P. R., and Jago, J. B. (Eds.), Antarctic Earth Science: Cambridge (Cambridge Univ. Press), 646-651.

Giret, A., Lameyre, J., Beaux, J.-F., Gautier, I., Verdier, O., Chotin, P., and Cantagrel, J.-M., 1987. Géologie, deux siècles de recherche dans les Iles Kerguelen. Actes Colloq. Rech. Fr. Terres Australes: 345355 .

Girod, M., and Nougier, J., 1972. Volcanism of the sub-Antarctic islands. In Adie, R. J. (Ed.), Antarctic Geology and Geophysics: Oslo (Universitetsforlaget), 777-789.

Gunn, B. M., Abranson, E. C., Watkins, N. D., and Nougier, J., 1972. Petrology and geochemistry of Iles Crozet: a summary. In Adie, R. J. (Ed.), Antarctic Geology and Geophysics: Oslo (Universitetsforlaget), 825-829.

Hayes, D. E., and Vogel, M., 1981. General Bathymetric Chart of the Oceans (GEBCO), Scale 1:10,000,000: Ottawa (Canadian Hydrographic Service), 5.13.

Houtz, R. E., Hayes, D. E., and Markl, R. G., 1977. Kerguelen Plateau bathymetry, sediment distribution and crustal structure. Mar. Geol., 25:95-130.

Hubberten, H.-W., Morche, W., Westall, F., Fütterer, D. K., and Keller, J., in press. Geochemical investigations of volcanic ash layers from Southern Atlantic ODP Legs 113 and 114. In Ciesielski, P. F., Kristoffersen, Y., et al., Proc. ODP, Sci. Results, 114: College Station, TX (Ocean Drilling Program).

Jarosewich, E., Nelen, J. A., and Norberg, J. A., 1980. Reference samples for electron microprobe analysis. Geostandards Newsl., 4:43-47.

Le Bas, M. J., Le Maitre, R. W., Streckeisen, A., and Zanettin, B., 1986. A chemical classification of volcanic rocks based on the Total Alkali-Silica Diagram. J. Petrol., 27:745-750.
Macdonald, G. A., and Katsura, T., 1964. Chemical composition of Hawaiian lavas. J. Petrol., 5:82-133.

Nougier, J., 1970. Contribution à l'étude géologique et géomorphique des iles Kerguelen. Com. Nat. Fr. Rech. Antarct., 27:1-440. 1972a. Geochronology of the volcanic activity in Iles Kerguelen. In Adie, R. J. (Ed.), Antarctic Geology and Geophysics: Oslo (Universitetsforlaget), 803-808.

1972b. Volcanic associations in Iles Kerguelen. In Adie, R. J. (Ed.), Antarctic Geology and Geophysics: Oslo (Universitetsforlaget), 809-815.

Nougier, J., Pawlowski, D., and Cantagrel, J. M., 1983. Chrono-spatial evolution of the volcanic activity in southeastern Kerguelen (T.A.A.F.). In Oliver, R. L., James, P. R., and Jago, J. B. (Eds.), Antarctic Earth Science: Cambridge (Cambridge Univ. Press), 640645.

Schlich, R., 1975. Structure et age de l'ocean Indien occidental. Mem. Hors Ser. Soc. Geol. Fr., 6:1-103.

Stephenson, P. J., 1972. Geochemistry of some Heard Island igneous rocks. In Adie, R. J. (Ed.), Antarctic Geology and Geophysics: Oslo (Universitetsforlaget), 793-801.

Watkins, N. D., Gunn, B. M., Nougier, J., and Baksi, A. K., 1974. Kerguelen: continental fragment or oceanic island. Geol. Soc. Am. Bull., $85: 201-212$.

Whitworth, T., III, 1988. The Antarctic circumpolar current. Oceanus, 31:53-58.

Date of initial receipt: 16 December 1989

Date of acceptance: 3 August 1990

Ms 119B-124

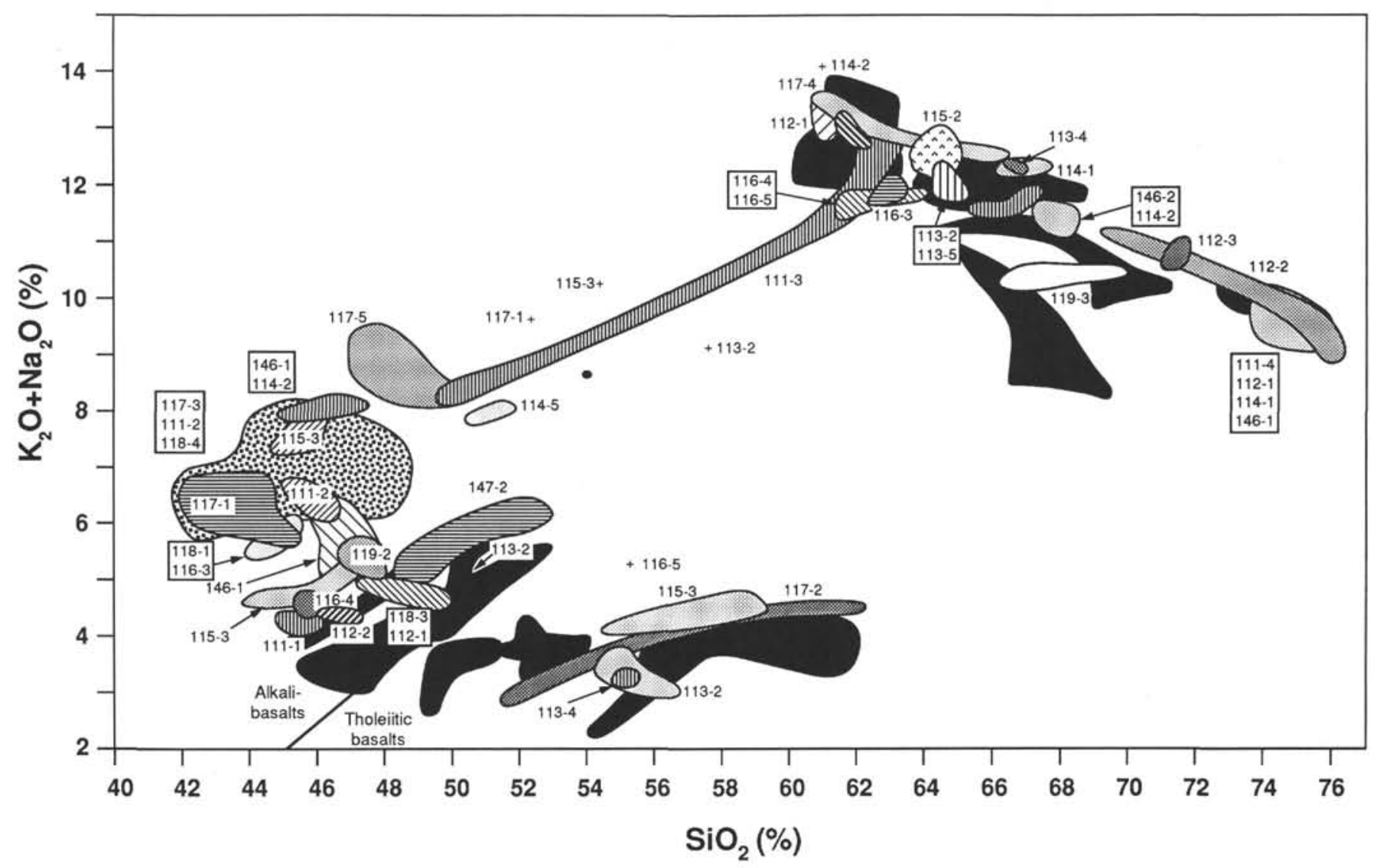

Figure 6. Total alkali vs. silica (TAS) diagram for the dispersed ashes from Sites 745 and 746 . Compositions of discrete tephra layers from Figure 5 are indicated in black for comparison. 
Table 5. Average composition of all Leg 119 calc-alkaline tephras (low-K-series) in comparison to South Sandwich Islands volcanic rocks and Quaternary Leg 114 tephra 701A-1H-4.

\begin{tabular}{|c|c|c|c|c|c|c|c|c|c|c|}
\hline & & $\begin{array}{c}\mathrm{SiO}_{2} \\
(\%)\end{array}$ & $\begin{array}{c}\mathrm{TiO}_{2} \\
(\%)\end{array}$ & $\begin{array}{c}\mathrm{Al}_{2} \mathrm{O}_{3} \\
(\%)\end{array}$ & $\begin{array}{l}\mathrm{FeO} \\
(\%)\end{array}$ & $\begin{array}{r}\mathrm{MnO} \\
(\%)\end{array}$ & $\begin{array}{r}\mathrm{MgO} \\
(\%)\end{array}$ & $\begin{array}{l}\mathrm{CaO} \\
(\%)\end{array}$ & $\begin{array}{l}\mathrm{K}_{2} \mathrm{O} \\
(\%)\end{array}$ & $\begin{array}{c}\mathrm{Na}_{2} \mathrm{O} \\
(\%)\end{array}$ \\
\hline Leg 119 & Mean & 56.87 & 1.12 & 15.40 & 10.96 & 0.20 & 3.25 & 8.49 & 0.58 & 3.03 \\
\hline $\mathrm{N}=24$ & SD & 2.90 & 0.21 & 0.98 & 1.40 & 0.11 & 1.46 & 1.28 & 0.18 & 0.54 \\
\hline S. Sandwich Is. ${ }^{a}$ & Mean & 55.50 & 1.00 & 16.13 & 10.96 & 0.19 & 3.85 & 8.70 & 0.55 & 3.00 \\
\hline$N=15$ & SD & 2.95 & 0.28 & 1.37 & 1.48 & 0.03 & 1.37 & 1.53 & 0.26 & 0.44 \\
\hline $\operatorname{Leg} 114^{b}$ & Mean & 56.64 & 1.09 & 15.56 & 11.17 & 0.25 & 3.41 & 8.27 & 0.36 & 3.20 \\
\hline $\mathrm{N}=15$ & $\mathrm{SD}$ & 0.79 & 0.10 & 0.41 & 0.62 & 0.09 & 0.43 & 0.25 & 0.06 & 0.32 \\
\hline
\end{tabular}

${ }^{a}$ Data from Baker (1978).

${ }^{b}$ Data from Hubberten et al. (in press).

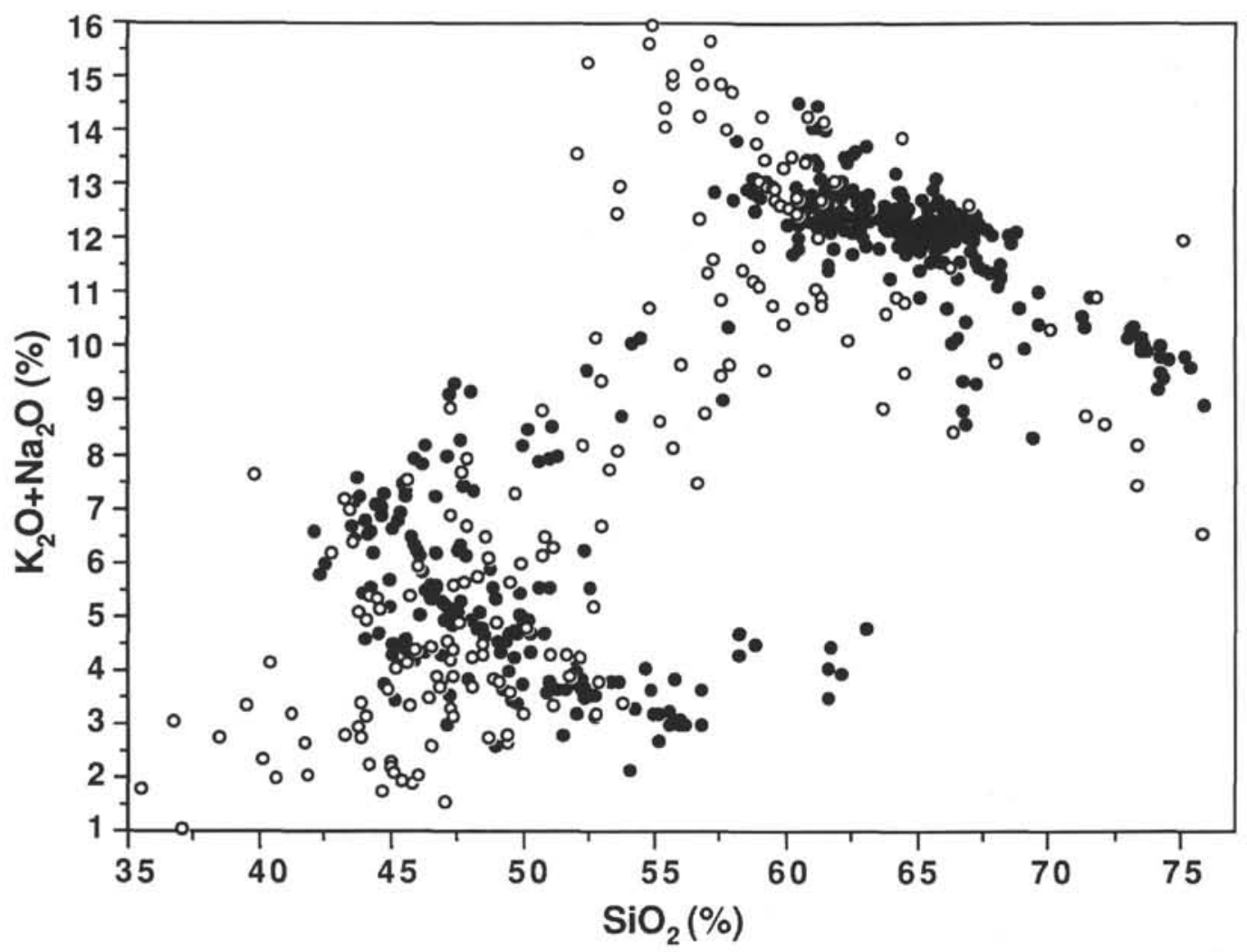

- Whole rock analyses Kerguelen (literature)

Analyses of glass

shards (this work)

Figure 7. Comparison of tephra geochemistry with whole-rock geochemistry from the Kerguelen Islands (Kerguelen data from Nougier, 1970). 


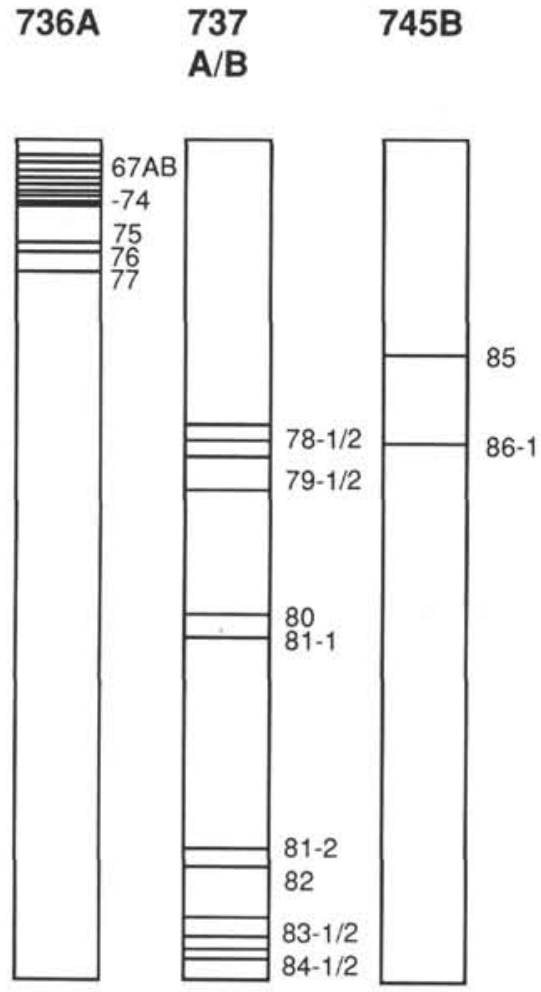

\section{Kerguelen}
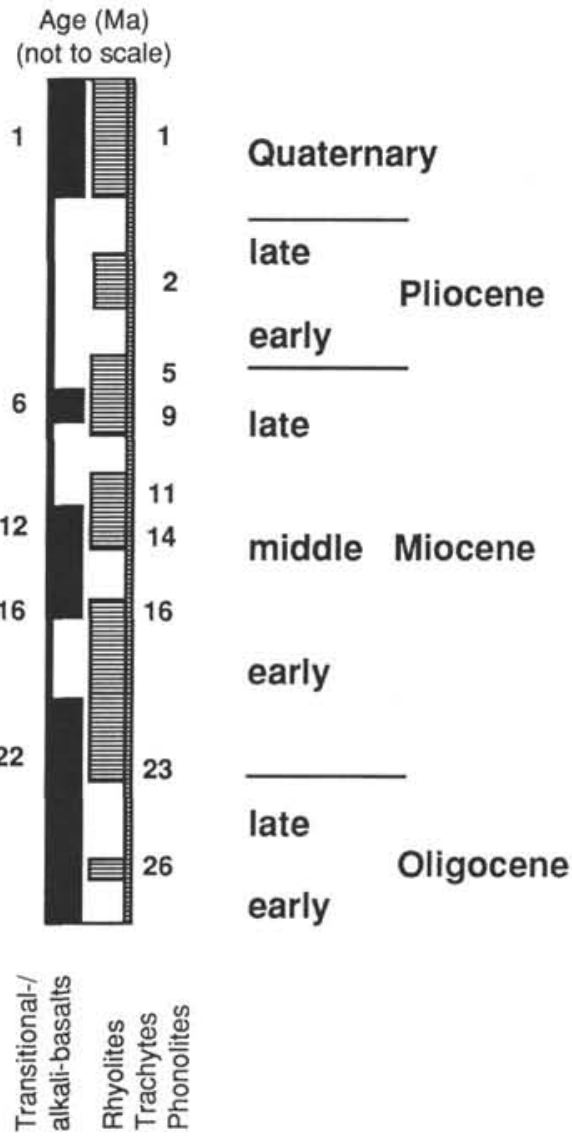

Figure 8. Schematic geochronological synopsis of discrete tephra layers from Leg 119 compared with dated rock series from the Kerguelen Islands (age determinations from Nougier, 1972a; Giret et al., 1987). 


\section{APPENDIX}

Major element analyses of all glass samples. Data set is normalized to $100 \%$ volatile-free with the exception for chlorine. Values of " 0.00 " are "below detection limit." Original sums vary in the range between $93 \%$ and $98 \%$ approximately, depending on volatile content and bubble-free surface measured.

\begin{tabular}{|c|c|c|c|c|c|c|c|c|c|c|c|}
\hline $\begin{array}{l}\text { AWI- } \\
\text { number }\end{array}$ & $\begin{array}{c}\mathrm{P}_{2} \mathrm{O}_{5} \\
(\%)\end{array}$ & $\begin{array}{c}\mathrm{SiO}_{2} \\
(\%)\end{array}$ & $\begin{array}{c}\mathrm{TiO}_{2} \\
(\%)\end{array}$ & $\begin{array}{c}\mathrm{Al}_{2} \mathrm{O}_{3} \\
(\%)\end{array}$ & $\begin{array}{l}\mathrm{FeO} \\
(\%)\end{array}$ & $\begin{array}{c}\mathrm{MnO} \\
(\%)\end{array}$ & $\begin{array}{c}\mathrm{MgO} \\
(\%)\end{array}$ & $\begin{array}{l}\mathrm{CaO} \\
(\%)\end{array}$ & $\begin{array}{l}\mathrm{K}_{2} \mathrm{O} \\
(\%)\end{array}$ & $\begin{array}{c}\mathrm{Na}_{2} \mathrm{O} \\
(\%)\end{array}$ & $\begin{array}{c}\mathrm{Cl} \\
(\%)\end{array}$ \\
\hline $67-1$ & 0.00 & 60.81 & 0.35 & 18.33 & 5.73 & 0.00 & 0.00 & 2.07 & 6.49 & 6.09 & \\
\hline $67-1$ & 0.00 & 61.71 & .38 & 18.70 & 4.47 & 0.00 & 0.00 & 1.71 & 6.17 & 6.69 & \\
\hline $67-1$ & 0.00 & 62.83 & 0.18 & 19.57 & 3.14 & 0.00 & 0.00 & 1.71 & 5.51 & 6.91 & \\
\hline $67-1$ & 0.00 & 65.18 & 0.48 & 16.14 & 4.95 & 0.00 & 0.00 & 1.00 & 5.45 & 6.58 & \\
\hline $67-1$ & 0.00 & 65.19 & 0.38 & 15.48 & 5.42 & 0.21 & 0.00 & 1.31 & 5.26 & 6.52 & \\
\hline $67-1$ & 0.00 & 65.69 & 0.00 & 14.79 & 4.80 & 0.00 & 0.00 & 1.29 & 5.11 & 7.89 & \\
\hline $67-1$ & 0.00 & 65.79 & 0.38 & 15.70 & 4.75 & 0.18 & 0.00 & 0.88 & & & \\
\hline $67-1$ & 0.00 & 65.80 & 0.36 & 15.94 & 4.32 & 0.00 & 0.00 & 0.82 & 5.16 & & \\
\hline $67-1$ & 0.00 & 65.83 & 0.40 & 16.38 & 4.12 & 0.00 & 0.00 & 1.02 & 5.45 & 6.61 & \\
\hline $67-1$ & 0.00 & 65.95 & 0.30 & 16.37 & 4.25 & 0.00 & 0.00 & 1.01 & 5.47 & & \\
\hline $67-1$ & 0.00 & 66.09 & 0.33 & 15.39 & 4.48 & 0.17 & 0.00 & 1.09 & 5.06 & 7.08 & \\
\hline $67-2$ & 0.00 & 60.52 & 36 & 18.1 & 4.97 & 0.00 & 0.00 & 1.38 & 6.62 & 7.74 & \\
\hline $67-2$ & 0.00 & 62.20 & 0.24 & 18. & 4.15 & 0.00 & 0.00 & 7 & .36 & 58 & \\
\hline $67-2$ & 0.00 & 62.37 & 0.33 & 19.18 & 3.68 & 0.00 & 0.0 & & .72 & 9 & \\
\hline $67-2$ & 0.00 & 63.13 & 0.14 & 18. & 4.00 & 0.00 & 0.0 & 1 & 7 & 22 & \\
\hline 67-2 & 0.00 & 63. & 0.69 & 17. & 4.01 & 0.00 & 0.0 & 6 & 0 & 7 & \\
\hline $67-2$ & $0: 00$ & 6 & .49 & 17. & 4.22 & 0.00 & 0. & & 3 & & \\
\hline $67-2$ & 0.00 & 64.62 & .46 & 17. & 3.94 & 0.00 & 0. & 9 & 0 & 2 & \\
\hline $67-2$ & 0.00 & 65.45 & 0.48 & 16.44 & 4.5 & 0.00 & 0. & 8 & .58 & 12 & \\
\hline $67-2$ & 0.00 & 65.49 & 0.31 & 16.51 & 4.2 & 0.00 & 0.00 & 1.07 & 5.43 & 6.81 & .1 \\
\hline $67-2$ & 0.00 & 65.85 & 0.37 & 16.24 & 4.36 & 0.00 & 0.00 & 1.06 & 5.42 & 6.42 & .2 \\
\hline $67-2$ & 0.00 & 66.07 & 0.28 & 16.85 & 3.42 & 0.00 & 0.00 & 0.84 & 5.22 & 7.17 & .1 \\
\hline $67-2$ & 0.00 & 6 & 0.36 & 14.59 & 4.99 & 0.19 & 0.0 & 0.87 & 4.87 & 7.62 & 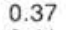 \\
\hline $67-2$ & 0.00 & 6 & 0.32 & 14.3 & 5.30 & 0.17 & 0.0 & 0.66 & 5.01 & 7.34 & \\
\hline $67-2$ & 0.00 & & 0.26 & 15 & 4.45 & 0.00 & 0.0 & 0.73 & 4.98 & 7.29 & \\
\hline 7.2 & & & & & 5.30 & 0.16 & 00 & 0.65 & 4.60 & 7.35 & \\
\hline 68 & 0.00 & 60.32 & 0.70 & & 3 & 0.00 & 0.00 & 2.62 & 6.21 & 5.41 & \\
\hline 68 & 0.00 & 60.58 & 0.61 & 18.39 & 5.85 & 0.00 & 0.00 & 2.58 & 6.18 & & \\
\hline 68 & 0.00 & 60.60 & 0.30 & 20.15 & 4.68 & 0.00 & 0.00 & 2.41 & 5.82 & 5.92 & \\
\hline 68 & 0.00 & 60.68 & 0.42 & 18.57 & 5.42 & 0.00 & 0.00 & 2.26 & 6.14 & 6.31 & \\
\hline 68 & 0.00 & 61.52 & 0.29 & 18.45 & 4.97 & 0.00 & 0.00 & 1.83 & 6.20 & 6.61 & \\
\hline 68 & 0.00 & 61.65 & 0.26 & 18.67 & 4.68 & 0.00 & 0.00 & 1.99 & 6.03 & 6.59 & \\
\hline 68 & 0.00 & 61.66 & 0.22 & 19.02 & 4.36 & 0.00 & 0.00 & 2.06 & 5.88 & 6.64 & \\
\hline 68 & 0.00 & 61.82 & 0.24 & 18.59 & 4.57 & 0.00 & 0.00 & 1.78 & 6.30 & 6.57 & \\
\hline 68 & 0.00 & 61.85 & 0.31 & 18.83 & 4.60 & 0.00 & 0.0 & 1.94 & 5.91 & & \\
\hline 68 & 0.00 & 6 & 0.25 & 18 & 4.10 & 0.00 & 0.0 & 2.11 & 6.32 & 6.37 & 0.1 \\
\hline 68 & 0.00 & 62 & .28 & 1 & 3.81 & 0.00 & 0.0 & 2.15 & 6.07 & & \\
\hline 68 & 0.00 & & .29 & 1 & 4. & 0.00 & & & 4 & 5 & \\
\hline 68 & 0.00 & & .38 & 16. & 3.72 & 0.00 & & 0.80 & 5.49 & & 0.2 \\
\hline 68 & 0.00 & & 0.32 & 16. & 3.73 & 0.00 & & 0.98 & 5.23 & 8 & \\
\hline$y$ & & & & & & 0. & & & & & \\
\hline 9 & 00 & & 2 & 8.14 & 0 & 0.00 & & & 4 & & \\
\hline 99 & 0.00 & & 6 & 1 & 3. & 0.00 & 0 & & 0 & & \\
\hline 99 & 0.00 & & 2 & 1 & 3. & 0.00 & & & 3 & & \\
\hline 39 & 0.00 & & 5 & 16 & 3 & 0.00 & & 8 & 9 & 1 & \\
\hline 70 & 0 & & 5 & & & 0 & & & & & \\
\hline 70 & 0.00 & & 30 & & 4.20 & 0.00 & & & & & \\
\hline 70 & 0.00 & & 28 & & 2.8 & 0.00 & & 4 & 1 & & \\
\hline 70 & 0.00 & & 15 & & 3.0 & 0.00 & & 1.30 & 0 & 8.25 & . \\
\hline 70 & 0.00 & & & & & 0.00 & & 4 & 3 & 5. & 0.0 \\
\hline 70 & 0.00 & & 8 & & & 0. & & 8 & 5.21 & 8.26 & 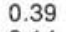 \\
\hline 70 & & & & & & & & 1.78 & 5.79 & 6.86 & 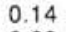 \\
\hline 70 & & & & & & & & 1.27 & 5.48 & 8.09 & 0.2 \\
\hline 70 & & & & & & 0.00 & & 1.89 & 4.38 & 8.19 & 0.1 \\
\hline 70 & 0.00 & & 5 & & & 0.00 & & 0.97 & 5.10 & 7.09 & 0.2 \\
\hline 70 & 0.00 & & & & & 0.00 & & 0.87 & 5. & & \\
\hline 70 & & & & 16 & & 0.00 & & 0.98 & 5.34 & 6.90 & .2 \\
\hline 71 & & & & & & & & & 5. & & 0.1 \\
\hline 71 & & & 26 & & & & & 7 & 6. & 6.66 & \\
\hline 71 & 0.00 & & .28 & 18. & & 0.00 & 0. & 2.30 & 5.81 & 6.87 & 0.1 \\
\hline 71 & & & 0.16 & 18 & & 0.00 & 0.00 & 2.30 & 6.07 & 6.40 & \\
\hline 71 & & & 0.20 & & & 0.00 & 0.00 & 2.39 & 5.96 & 6.24 & 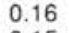 \\
\hline 71 & & & 0.25 & & & 0.00 & 0.00 & 1.94 & 5.80 & 7.17 & . \\
\hline 71 & & & 0.22 & & & 0.00 & 0.00 & 2.25 & 6.14 & 6.23 & 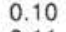 \\
\hline 71 & 0.00 & & 0.22 & 18.56 & & 0.00 & 0.00 & 2.21 & 5.77 & 6.55 & 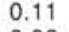 \\
\hline 71 & 0.00 & 62.71 & 0.00 & 18.77 & 4.09 & 0.00 & 0.00 & 2.31 & 6.03 & 6.03 & \\
\hline
\end{tabular}


Appendix (continued).

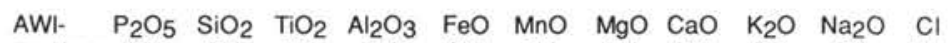

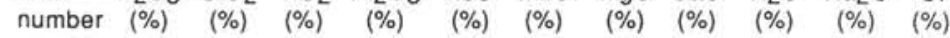

\begin{tabular}{|c|c|c|c|c|c|c|c|c|c|c|c|}
\hline 72 & 0.00 & 62.18 & 0.00 & 18.21 & 5.03 & 0.00 & 0.00 & 1.65 & 6.79 & 5.95 & 0.19 \\
\hline 72 & 0.00 & 62.76 & 0.13 & 18.66 & 4.01 & 0.00 & 0.00 & 1.57 & 6.20 & 6.45 & 0.22 \\
\hline 72 & 0.00 & 65.02 & 0.56 & 16.61 & 4.91 & 0.00 & 0.00 & 0.94 & 5.79 & 6.06 & 0.11 \\
\hline 72 & 0.00 & 65.04 & 0.47 & 16.74 & 4.31 & 0.00 & 0.00 & 1.13 & 5.94 & 6.23 & 0.13 \\
\hline 72 & 0.00 & 65.16 & 0.42 & 16.96 & 3.99 & 0.00 & 0.00 & 1.18 & 6.19 & 5.95 & 0.15 \\
\hline 72 & 0.00 & 65.49 & 0.39 & 16.37 & 4.41 & 0.00 & 0.00 & 1.08 & 5.72 & 6.43 & 0.11 \\
\hline 72 & 0.00 & 65.50 & 0.36 & 16.69 & 4.14 & 0.00 & 0.00 & 1.13 & 5.92 & 6.13 & 0.13 \\
\hline 72 & 0.00 & 65.62 & 0.42 & 16.48 & 4.33 & 0.00 & 0.00 & 1.09 & 5.82 & 5 & 0.20 \\
\hline 72 & 0.00 & 65.78 & 0.37 & 16.00 & 4.89 & 0.00 & 0.00 & 0.90 & 5.32 & & 0.18 \\
\hline 72 & 0.00 & 66.30 & 0.27 & 15.90 & 4.29 & 0.00 & 0.00 & 1.03 & 5.55 & 0 & 0 \\
\hline 3 & 0.45 & 53.95 & 2.57 & 17.09 & 8.50 & 0.00 & 1.95 & 6.63 & 4.53 & 4.17 & 0.17 \\
\hline 3 & 0.00 & 61.20 & 0.20 & 18.22 & 5.78 & 0.00 & 0.00 & 1.89 & 6.50 & 10 & 0.12 \\
\hline 3 & 0.00 & 61.90 & 0.14 & 18 & 4.72 & 0.00 & 0.00 & 1.70 & 6 & 61 & 0 \\
\hline 3 & 0.00 & 62.22 & 0.12 & 18 & 4.13 & 0.14 & 0.00 & 1.25 & 5.83 & 7.53 & 0 \\
\hline 3 & 0.00 & 62.35 & 0.00 & 18 & 3.99 & 0.00 & 0.00 & 1.13 & 6 & 1 & 0 \\
\hline 3 & 0.00 & 62.53 & 0.28 & 18 & 4.07 & 0.00 & 0.00 & 1.68 & 6.10 & 6.63 & 0.26 \\
\hline 3 & 0.00 & 62.72 & 0.28 & 18 & 4.15 & 0.00 & 0.00 & 1.81 & 5.92 & 40 & 0.25 \\
\hline 3 & 0.00 & 65 & 0.38 & 16 & 4. & 0.00 & 0.00 & 1.13 & 5.62 & 6.61 & 0.20 \\
\hline 3 & 0.00 & 65.34 & 0.54 & 69 & 3.82 & 0.00 & 0.00 & 0.99 & 6.16 & .29 & 0.18 \\
\hline 3 & 0.00 & 66 & 0.31 & 28 & 4. & 0.00 & 0.00 & 1.12 & 5.65 & 6.21 & 0.16 \\
\hline 3 & 0.00 & 66.35 & 0.34 & 15.30 & 4.69 & 0.00 & 0.00 & 0.80 & 5.06 & 7.12 & \\
\hline 4 & 0.00 & 65.76 & 0.44 & 16.69 & 4.03 & 0.00 & 0.00 & 0.94 & 5.75 & 6.24 & 0.17 \\
\hline 4 & 0.00 & 65.99 & 0.65 & 16.59 & 3.89 & 0.00 & 0.00 & 1.09 & 5.95 & 5.85 & 0.00 \\
\hline 4 & 0.00 & 66.02 & 1.01 & 16.13 & 3.55 & 0.00 & 0.00 & 0.74 & 5.59 & 6.69 & 0.27 \\
\hline 4 & 0.00 & 66.17 & 0.40 & 15.89 & 4.50 & 0.00 & 0.00 & 0.93 & 5.51 & & 0.09 \\
\hline 4 & 0.00 & 66.72 & 0.49 & 16.80 & 3.07 & 0.00 & 0.00 & 0.74 & 6.12 & 8 & 0.11 \\
\hline 74 & 0.00 & 66.99 & 0.41 & 16.29 & 3.19 & 0.00 & 0.00 & 0.60 & 5.66 & 33 & 0.23 \\
\hline 4 & 0.00 & 67.15 & 0.41 & 15.15 & 3.95 & 0.16 & 0.00 & 0.92 & 5.13 & 22 & 0.20 \\
\hline 74 & 0.00 & 2 & 0.36 & 12 & 4.42 & 0.00 & 0.00 & 0.47 & 4. & 7.17 & 0.36 \\
\hline 75 & 0.00 & 64.39 & 0.35 & 16.20 & 5.09 & 0.00 & 0.00 & 1.07 & 5.57 & 7.17 & 0.16 \\
\hline 75 & 0.00 & 65.51 & 0.41 & 15.75 & 4.97 & 0.00 & 0.00 & 1.05 & 5.36 & 6.75 & 0.19 \\
\hline 75 & 0.00 & 65.52 & 0.37 & 15.84 & 4.90 & 0.00 & 0.00 & 1.29 & 5.47 & 6.43 & 0.19 \\
\hline 75 & 0.00 & 65.64 & 0.58 & 17.25 & 2.81 & 0.14 & 0.00 & 0.90 & 6.25 & 6.25 & 0.17 \\
\hline 75 & 0.00 & 65.64 & 0.42 & 16.45 & 4.26 & 0.00 & 0.00 & 0.96 & 5.59 & 6.48 & 0.20 \\
\hline 75 & 0.00 & 66.10 & 0.35 & 14.80 & 5.35 & 0.00 & 0.00 & 1.06 & 5.21 & 6. & 0.32 \\
\hline 75 & 0.00 & 66.87 & 0.53 & 16.65 & 2.80 & 0.00 & 0.00 & 0.68 & 5.94 & 6.33 & 0.19 \\
\hline 75 & 0.00 & 66.95 & 0.45 & 16.67 & 2.93 & 0.00 & 0.00 & 0.88 & 6.08 & 5.90 & 0.14 \\
\hline 75 & 0.00 & 68.43 & 0.43 & 15.00 & 3.41 & 0.00 & 0.00 & 0.57 & 5.54 & 6.29 & 0.33 \\
\hline 75 & .00 & 68.66 & 0.42 & 14 & 3.36 & 0.00 & 0.00 & 0.43 & 5.54 & 6.46 & 0.28 \\
\hline 76 & 0.00 & 61.30 & 0.19 & 18 & 4.30 & 0.00 & 0.00 & 1.20 & 6.19 & 8.13 & 0.28 \\
\hline 76 & 0.00 & 64.34 & 0.54 & 18.09 & 2.98 & 0.00 & 0.00 & 1.59 & 7.20 & 5.17 & 0.08 \\
\hline 76 & 0.00 & 64.81 & 0.52 & 16.46 & 4.83 & 0.00 & 0.00 & 1.31 & 5.71 & 6.23 & 0.13 \\
\hline 76 & 0.00 & 64.96 & 0.87 & 17.49 & 3.27 & 0.00 & 0.00 & 1.17 & 6.54 & 5.63 & 0.07 \\
\hline 76 & 0.00 & 65.13 & 0.52 & 16.03 & 4.83 & 0.00 & 0.00 & 1.23 & 5.61 & 6.50 & 0.15 \\
\hline 76 & 0.00 & 65.37 & 0.44 & 15.73 & 5.05 & 0.00 & 0.00 & 1.13 & 5.43 & 6.62 & 0.23 \\
\hline 76 & 0.00 & 65.42 & 0.39 & 16 & 4.49 & 0.00 & 0.00 & 1.17 & 5 & 6.35 & 0.17 \\
\hline 76 & 0.00 & 65.83 & 0.53 & 15 & 4.82 & 0.13 & 0.00 & 1.07 & 5.63 & 6.17 & 0.18 \\
\hline 76 & 0.00 & 66.49 & 0.22 & 15 & 4.41 & 0.00 & 0.00 & 0.77 & 5.13 & 6.91 & 0.43 \\
\hline 76 & 0.00 & 68.35 & 0.52 & 14.43 & 3.75 & 0.21 & 0.00 & 0.47 & 5.48 & 6.47 & 0.32 \\
\hline 7 & 0.70 & 52.54 & 2.89 & 14.38 & 2.39 & 0.30 & 2.80 & 7.60 & 2.07 & 4.22 & 0.10 \\
\hline 7 & 0.00 & 54.24 & 0.90 & 15.62 & 11.07 & 0.00 & 5.08 & 10.78 & 0.38 & 1.93 & 0.00 \\
\hline 77 & 0.00 & 55.79 & 0.94 & 14.94 & 12.27 & 0.00 & 3.76 & 8.90 & 0.60 & 2.80 & 0.00 \\
\hline 77 & 0.00 & 55.90 & 1.06 & 17.66 & 9.72 & 0.18 & 2.40 & 8.99 & 0.54 & 3.43 & 0.12 \\
\hline 77 & 0.00 & 56.14 & 1.20 & 14.72 & 12.52 & 0.00 & 3.40 & 8.80 & 0.36 & 2.76 & 0.09 \\
\hline 77 & 0.00 & 56.97 & 1.23 & 14.65 & 12.14 & 0.18 & 2.76 & 8.08 & 0.63 & 3.16 & 0.19 \\
\hline 7 & 0.00 & 61.66 & 1.29 & 15.98 & 6.64 & 0.14 & 1.58 & 8.50 & 0.47 & 3.68 & 0.06 \\
\hline 77 & 0.00 & 61.67 & 1.00 & 14.48 & 10.48 & 0.15 & 1.38 & 7.12 & 0.53 & 3.07 & 0.12 \\
\hline 77 & 0.00 & 63.80 & 0.65 & 17.54 & 4.12 & 0.00 & 0.00 & 1.72 & 6.73 & 5.37 & 0.06 \\
\hline 77 & 0.00 & 64.01 & 0.43 & 16.27 & 5.53 & 0.18 & 0.00 & 1.27 & 5.51 & 6.70 & 0.11 \\
\hline 77 & 0.00 & 65.15 & 0.36 & 16.07 & 4.76 & 0.00 & 0.00 & 0.84 & 5.48 & 7.11 & 0.22 \\
\hline 77 & 0.00 & 66.85 & 0.45 & 16.82 & 3.19 & 0.00 & 0.00 & 0.76 & 5.75 & 6.18 & 0.00 \\
\hline 1 & 0.00 & 67.20 & 0.56 & 14.54 & 4.62 & 0.00 & 0.00 & 0.51 & 5.32 & 6.99 & 0.27 \\
\hline 8. & 0.0 & & 0.88 & & 4.25 & 0.00 & 0.00 & 1.72 & 6.69 & 5.38 & 0.06 \\
\hline 78 & 0.00 & 65.64 & 0.59 & 15.81 & 4.76 & 0.00 & 0.00 & 0.95 & 5.54 & 6.60 & 0.12 \\
\hline 78 & 0.00 & 65.66 & 0.49 & 15.90 & 4.77 & 0.00 & 0.00 & 1.38 & 6.04 & 5.63 & 0.12 \\
\hline 78 & 0.00 & 65.79 & 0.43 & 15.20 & 5.46 & 0.19 & 0.00 & 0.83 & 5.03 & 6.87 & 0.21 \\
\hline $78-1$ & 0.00 & 65.83 & 0.46 & 16.35 & 4.42 & 0.17 & 0.00 & 1.15 & 6.19 & 5.30 & 0.13 \\
\hline $78-1$ & 0.00 & 66.32 & 0.39 & 14.50 & 5.55 & 0.00 & 0.00 & 0.67 & 4.94 & 7.45 & 0.19 \\
\hline $78-1$ & 0.00 & 66.33 & 0.41 & 14.57 & 5.44 & 0.00 & 0.00 & 0.59 & 4.99 & 7.39 & 0.26 \\
\hline 78-1 & 0.00 & 66.42 & 0.31 & 14.32 & 5.51 & 0.00 & 0.00 & 0.77 & 5.38 & 6.99 & 0.30 \\
\hline $78-1$ & 0.00 & 66.92 & 0.49 & 13.26 & 5.96 & 0.18 & 0.00 & 0.54 & 4.77 & 7.56 & 0.32 \\
\hline $8-1$ & 0.00 & 67.08 & 0.44 & 13.06 & 6.06 & 0.18 & 0.00 & 0.51 & 4.68 & 7.67 & 0.32 \\
\hline 8-1 & 0.00 & 67.20 & 0.43 & 13.26 & 5.97 & 0.14 & 0.00 & 0.53 & 4.79 & 7.31 & 0.37 \\
\hline
\end{tabular}


Appendix (continued).

\begin{tabular}{|c|c|c|c|c|c|c|c|c|c|c|c|}
\hline $\begin{array}{l}\text { AWI- } \\
\text { number }\end{array}$ & $\begin{array}{c}\mathrm{P}_{2} \mathrm{O}_{5} \\
(\%)\end{array}$ & $\begin{array}{c}\mathrm{SiO}_{2} \\
(\%)\end{array}$ & $\begin{array}{c}\mathrm{TiO}_{2} \\
(\%)\end{array}$ & $\begin{array}{c}\mathrm{Al}_{2} \mathrm{O}_{3} \\
(\%)\end{array}$ & $\begin{array}{l}\mathrm{FeO} \\
(\%)\end{array}$ & $\begin{array}{c}\mathrm{MnO} \\
(\%)\end{array}$ & $\begin{array}{c}\mathrm{MgO} \\
(\%)\end{array}$ & $\begin{array}{l}\mathrm{CaO} \\
(\%)\end{array}$ & $\begin{array}{l}\mathrm{K}_{2} \mathrm{O} \\
(\%)\end{array}$ & $\begin{array}{c}\mathrm{Na}_{2} \mathrm{O} \\
(\%)\end{array}$ & $\begin{array}{l}\mathrm{Cl} \\
(\%)\end{array}$ \\
\hline $78-2$ & 0.00 & 66.21 & 0.35 & 15 & 5.04 & 0.18 & 0.00 & 0.71 & 5.33 & 6.92 & 0.21 \\
\hline $78-2$ & 0.00 & 66.38 & 0.45 & 14.78 & 5.31 & 0.18 & 0.00 & 0.73 & 5.40 & 6.46 & 0.31 \\
\hline $78-2$ & 0.00 & 66.84 & 0.50 & 14.52 & 5.21 & 0.00 & 0.00 & 0.59 & 5.21 & 6.81 & 0.32 \\
\hline $78-2$ & 0.00 & 66.85 & 0.38 & 14.32 & 5.14 & 0.16 & 0.00 & 0.65 & 4.99 & 7.16 & 0.37 \\
\hline $78-2$ & 0.00 & 66.93 & 0.30 & 14.29 & 5.27 & 0.23 & 0.00 & 0.67 & 5.01 & 7.03 & 0.26 \\
\hline $78-2$ & 0.00 & 67.03 & 0.39 & 14.15 & 5.37 & 0.16 & 0.00 & 0.64 & 5.03 & 6.91 & 0.33 \\
\hline $78-2$ & 0.00 & 67.03 & 0.48 & 14.03 & 5.42 & 0.16 & 0.00 & 0.66 & 5.16 & 6.77 & 0.30 \\
\hline $78-2$ & 0.00 & 67.06 & 0.39 & 14.10 & 5.49 & 0.00 & 0.00 & 0.57 & 4.90 & 7.16 & 0.34 \\
\hline $78-2$ & 0.00 & 67.08 & 0.41 & 14.20 & 5.39 & 0.15 & 0.00 & 0.64 & 5.25 & 6.62 & 0.28 \\
\hline $78-2$ & 0.00 & 67.12 & 0.44 & 14.19 & 5.21 & 0.17 & 0.00 & 0.54 & 5.01 & 7.07 & 0.25 \\
\hline $79-1$ & 0.00 & 64.37 & 0.35 & 16.40 & 5.26 & 0.00 & 0.00 & 1.37 & 5.79 & 6.26 & 0.20 \\
\hline $79-1$ & 0.00 & 64.38 & 0.37 & 16.48 & 5.13 & 0.00 & 0.00 & 1.46 & 5.83 & 6.23 & 0.13 \\
\hline $79-1$ & 0.00 & 64.53 & 0.45 & 16.04 & 5.54 & 0.16 & 0.00 & 1.33 & 5.53 & 6.19 & 0.25 \\
\hline $79-1$ & 0.00 & 64.59 & 0.38 & 16.31 & 5.30 & 0.00 & 0.00 & 1.33 & 5.79 & 6.11 & 0.20 \\
\hline $79-1$ & 0.00 & 64.61 & 0.26 & 16.01 & 5.54 & 0.00 & 0.00 & 1.32 & 5.77 & 6.32 & 0.17 \\
\hline $79-1$ & 0.00 & 65.04 & 0.36 & 16.42 & 5.23 & 0.00 & 0.00 & 1.47 & 5.59 & 5.73 & 0.17 \\
\hline $79-2$ & 0.00 & 63.96 & 1.03 & 16.10 & 5.37 & 0.18 & 0.00 & 2.15 & 5.63 & 5.58 & 0.00 \\
\hline $79-2$ & 0.00 & 66.47 & 0.74 & 15.49 & 4.80 & 0.00 & 0.00 & 1.20 & 5.47 & 5.74 & 0.08 \\
\hline $79-2$ & 0.00 & 66.55 & 0.64 & 15.54 & 4.64 & 0.00 & 0.00 & 1.06 & 5.82 & 5.65 & 0.09 \\
\hline $79-2$ & 0.00 & 67.16 & 0.57 & 14.90 & 4.78 & 0.00 & 0.00 & 0.96 & 5.60 & 5.88 & 0.16 \\
\hline $79-2$ & 0.00 & 67.27 & 0.53 & 14.41 & 4.97 & 0.24 & 0.00 & 0.98 & 5.55 & 5.83 & 0.23 \\
\hline $79-2$ & 0.00 & 67.69 & 0.49 & 14.62 & 4.75 & 0.00 & 0.00 & 0.99 & 5.55 & 5.73 & 0.18 \\
\hline $79-2$ & 0.00 & 67.96 & 0.40 & 14.44 & 4.93 & 0.14 & 0.00 & 0.94 & 5.38 & 5.66 & 0.18 \\
\hline $79-2$ & 0.00 & 68.06 & 0.44 & 14.47 & 4.87 & 0.00 & 0.00 & 0.83 & 5.72 & 5.45 & 0.15 \\
\hline $79-2$ & 0.00 & 68.11 & 0.47 & 14.34 & 4.97 & 0.00 & 0.00 & 0.69 & 5.29 & 5.96 & 0.18 \\
\hline $79-2$ & 0.00 & 68.83 & 0.43 & 14.48 & 4.50 & 0.00 & 0.00 & 0.85 & 5.64 & 5.04 & 0.22 \\
\hline $79-2$ & 0.00 & 69.02 & 0.48 & 14.68 & 4.83 & 0.00 & 0.00 & 0.86 & 5.49 & 4.45 & 0.19 \\
\hline $79-2$ & 0.00 & 71.19 & 0.35 & 11.77 & 5.58 & 0.00 & 0.00 & 0.48 & 5.21 & 5.13 & 0.29 \\
\hline 80 & 0.00 & 72.84 & 0.22 & 13.22 & 2.72 & 0.00 & 0.00 & 0.49 & 5.01 & 5.10 & 0.40 \\
\hline 80 & 0.00 & 72.86 & 0.19 & 13.19 & 2.73 & 0.00 & 0.00 & 0.41 & 5.07 & 5.21 & 0.34 \\
\hline 80 & 0.00 & 72.96 & 0.14 & 13.37 & 2.51 & 0.00 & 0.00 & 0.41 & 5.13 & 5.18 & 0.31 \\
\hline 80 & 0.00 & 73.25 & 0.00 & 13.18 & 2.73 & 0.00 & 0.00 & 0.35 & 5.29 & 4.84 & 0.35 \\
\hline 80 & 0.00 & 73.26 & 0.20 & 13.13 & 2.84 & 0.00 & 0.00 & 0.34 & 5.12 & 4.76 & 0.34 \\
\hline 80 & 0.00 & 73.27 & 0.22 & 13.23 & 2.52 & 0.00 & 0.00 & 0.40 & 4.96 & 5.03 & 0.37 \\
\hline 80 & 0.00 & 73.35 & 0.16 & 12.97 & 2.77 & 0.00 & 0.00 & 0.38 & 4.95 & 5.04 & 0.38 \\
\hline 80 & 0.00 & 73.54 & 0.00 & 13.15 & 2.66 & 0.00 & 0.00 & 0.37 & 5.09 & 4.81 & 0.36 \\
\hline 80 & 0.00 & 74.89 & 0.14 & 12.28 & 2.30 & 0.00 & 0.00 & 0.27 & 5.57 & 4.24 & 0.32 \\
\hline $81-1$ & 0.00 & 65.08 & 0.76 & 17.22 & 4.49 & 0.00 & 0.00 & 1.46 & 5.58 & 5.29 & 0.12 \\
\hline $81-1$ & 0.00 & 65.77 & 0.39 & 14.47 & 5.69 & 0.00 & 0.00 & 0.78 & 5.08 & 7.50 & 0.32 \\
\hline $81-1$ & 0.00 & 66.05 & 0.40 & 16.14 & 5.51 & 0.00 & 0.00 & 1.07 & 4.99 & 5.66 & 0.18 \\
\hline $81-1$ & 0.00 & 66.23 & 0.63 & 17.45 & 4.10 & 0.00 & 0.00 & 1.45 & 4.97 & 5.08 & 0.10 \\
\hline $81-1$ & 0.00 & 66.68 & 0.63 & 17.45 & 4.81 & 0.00 & 0.00 & 1.53 & 4.47 & 4.36 & 0.07 \\
\hline $81-1$ & 0.00 & 66.72 & 0.51 & 16.57 & 5.33 & 0.15 & 0.00 & 1.19 & 4.49 & 4.87 & 0.16 \\
\hline $81-1$ & 0.00 & 66.79 & 0.71 & 17.55 & 4.85 & 0.00 & 0.00 & 1.42 & 4.10 & 4.49 & 0.08 \\
\hline $81-1$ & 0.00 & 67.13 & 0.56 & 16.52 & 5.18 & 0.00 & 0.00 & 1.17 & 4.55 & 4.73 & 0.17 \\
\hline $81-1$ & 0.00 & 67.92 & 0.43 & 14.56 & 6.02 & 0.15 & 0.00 & 0.73 & 4.78 & 4.95 & 0.46 \\
\hline $81-1$ & 0.00 & 69.28 & 0.47 & 14.48 & 6.27 & 0.00 & 0.00 & 0.78 & 4.16 & 4.18 & 0.39 \\
\hline $81-2$ & 0.00 & 50.23 & 3.68 & 15.28 & 12.74 & 0.20 & 3.28 & 9.58 & 1.90 & 3.12 & 0.00 \\
\hline $81-2$ & 0.00 & 52.26 & 3.75 & 13.14 & 14.52 & 0.16 & 3.53 & 8.78 & 1.33 & 2.54 & 0.00 \\
\hline $81-2$ & 0.00 & 52.29 & 3.58 & 13.43 & 14.74 & 0.20 & 3.60 & 8.82 & 1.09 & 2.25 & 0.00 \\
\hline $81-2$ & 0.00 & 52.43 & 3.62 & 13.49 & 13.69 & 0.21 & 3.70 & 8.89 & 1.14 & 2.82 & 0.00 \\
\hline $81-2$ & 0.00 & 52.99 & 3.34 & 13.70 & 13.23 & 0.19 & 3.82 & 9.45 & 0.83 & 2.45 & 0.00 \\
\hline $81-2$ & 0.00 & 53.55 & 3.77 & 13.55 & 13.58 & 0.15 & 3.05 & 8.44 & 1.08 & 2.83 & 0.00 \\
\hline $81-2$ & 0.00 & 53.88 & 3.59 & 13.68 & 13.12 & 0.22 & 3.33 & 8.26 & 1.19 & 2.73 & 0.00 \\
\hline 82 & 0.00 & 51.56 & 3.65 & 13.26 & 14.6 & 0.00 & 3. & 9.45 & 1.13 & 2.62 & 0.00 \\
\hline 82 & 0.00 & 51.88 & 3.52 & 13.48 & 14.05 & 0.17 & 3.8 & 9.02 & 1.16 & 2.63 & 0.25 \\
\hline 82 & 0.00 & 52.10 & 3.56 & 13.50 & 13.72 & 0.14 & 3.97 & 9.09 & 1.18 & 2.74 & 0.00 \\
\hline 82 & 0.00 & 52.16 & 3.71 & 13.24 & 14.42 & 0.15 & 3.57 & 8.74 & 1.29 & 2.71 & 0.00 \\
\hline 82 & 0.00 & 52.17 & 3.62 & 13.54 & 13.69 & 0.18 & 3.77 & 8.99 & 1.18 & 2.86 & 0.00 \\
\hline 82 & 0.00 & 52.21 & 3.45 & 13.45 & 13.73 & 0.16 & 3.88 & 8.85 & 1.36 & 2.92 & 0.00 \\
\hline 82 & 0.00 & 52.22 & 3.49 & 13.40 & 13.82 & 0.18 & 3.84 & 8.89 & 1.12 & 2.97 & 0.06 \\
\hline 82 & 0.00 & 52.27 & 3.59 & 13.88 & 13.78 & 0.00 & 3.67 & 8.93 & 1.01 & 2.87 & 0.00 \\
\hline 82 & 0.00 & 52.27 & 3.59 & 13.57 & 13.73 & 0.00 & 3.91 & 8.86 & 1.05 & 3.02 & 0.00 \\
\hline 82 & 0.00 & 52.46 & 3.43 & 13.51 & 13.86 & 0.00 & 3.86 & 9.11 & 1.14 & 2.62 & 0.00 \\
\hline 82 & 0.00 & 52.48 & 3.68 & 13.50 & 14.13 & 0.16 & 3.58 & 8.69 & 1.18 & 2.59 & 0.00 \\
\hline 82 & 0.00 & 52.52 & 3.45 & 13.68 & 13.70 & 0.00 & 3.74 & 9.15 & 1.08 & 2.68 & 0.00 \\
\hline 82 & 0.00 & 52.53 & 3.55 & 13.82 & 13.60 & 0.14 & 3.77 & 8.96 & 1.15 & 2.49 & 0.00 \\
\hline 82 & 0.00 & 52.54 & 3.65 & 13.31 & 13.81 & 0.18 & 3.71 & 9.08 & 1.16 & 2.56 & 0.00 \\
\hline 82 & 0.00 & 52.55 & 3.41 & 13.51 & 13.65 & 0.18 & 3.84 & 9.04 & 1.13 & 2.69 & 0.00 \\
\hline 82 & 0.00 & 52.83 & 3.47 & 13.53 & 13.79 & 0.00 & 3.82 & 8.89 & 1.06 & 2.61 & 0.00 \\
\hline 82 & 0.00 & 52.91 & 3.67 & 13.68 & 13.82 & 0.00 & 4.04 & 8.21 & 1.22 & 2.45 & 0.00 \\
\hline
\end{tabular}


Appendix (continued).

\begin{tabular}{|c|c|c|c|c|c|c|c|c|c|c|c|}
\hline 1- & & $\begin{array}{l}\mathrm{iO}_{2} \\
(\%)\end{array}$ & $\begin{array}{l}\mathrm{O}_{2} \\
\%)\end{array}$ & ${ }_{2} \mathrm{O}_{3}$ & $\begin{array}{l}\mathrm{FeO} \\
(\%)\end{array}$ & $\begin{array}{l}\text { InO } \\
\%)\end{array}$ & $\begin{array}{l}\mathrm{lgO} \\
\%)\end{array}$ & $\begin{array}{l}\mathrm{CaO} \\
(\%)\end{array}$ & $\begin{array}{l}2 \mathrm{O} \\
\%)\end{array}$ & $\begin{array}{l}\mathrm{Na}_{2} \mathrm{O} \\
(\%)\end{array}$ & \\
\hline-1 & $\begin{array}{l}00 \\
00 \\
00 \\
00 \\
00 \\
00\end{array}$ & $\begin{array}{l}49.25 \\
49.50 \\
49.86 \\
49.86 \\
50.28 \\
51.17\end{array}$ & $\begin{array}{l}3.82 \\
3.76 \\
3.38 \\
3.78 \\
3.04 \\
2.70 \\
4.01\end{array}$ & $\begin{array}{l}12.62 \\
13.95 \\
13.98 \\
14.38 \\
14.86 \\
15.11 \\
14.61\end{array}$ & $\begin{array}{l}15.92 \\
14.09 \\
13.00 \\
14.02 \\
12.13 \\
12.04 \\
14.33\end{array}$ & $\begin{array}{l}0.30 \\
0.74 \\
0.43 \\
0.52 \\
0.69 \\
0.37 \\
0.37\end{array}$ & $\begin{array}{l}91 \\
20 \\
23 \\
.09 \\
60 \\
53 \\
95\end{array}$ & $\begin{array}{r}10.45 \\
10.00 \\
10.57 \\
9.77 \\
10.52 \\
10.34 \\
7.60\end{array}$ & $\begin{array}{l}0.35 \\
0.60 \\
0.46 \\
0.36 \\
0.44 \\
0.45 \\
0.61\end{array}$ & $\begin{array}{l}2.38 \\
3.16 \\
3.10 \\
3.22 \\
3.43 \\
3.29 \\
3.28\end{array}$ & $\begin{array}{l}0.00 \\
0.00 \\
0.00\end{array}$ \\
\hline & $\begin{array}{l}00 \\
00 \\
00 \\
00 \\
00 \\
00 \\
00 \\
00\end{array}$ & $\begin{array}{l}49.91 \\
49.93 \\
50.06 \\
50.17\end{array}$ & $\begin{array}{l}3.75 \\
3.58 \\
3.46 \\
3.74 \\
4.14 \\
3.43 \\
3.80 \\
3.36\end{array}$ & $\begin{array}{l}14.66 \\
15.08 \\
14.84 \\
14.66 \\
15.51 \\
15.30 \\
14.99 \\
15.36\end{array}$ & $\begin{array}{l}13.28 \\
12.74 \\
12.90 \\
14.36 \\
12.18 \\
13.35 \\
12.31 \\
11.92\end{array}$ & & $\begin{array}{l}3.84 \\
.78\end{array}$ & $\begin{array}{l}9.22 \\
9.98 \\
9.55 \\
8.34 \\
8.75 \\
8.47 \\
8.68 \\
9.06\end{array}$ & $\begin{array}{l}0.91 \\
1.20 \\
1.22 \\
1.52 \\
1.68 \\
0.78 \\
1.97 \\
1.22\end{array}$ & $\begin{array}{l}3.94 \\
3.17 \\
3.58 \\
3.60 \\
3.84 \\
3.99 \\
3.66 \\
3.55\end{array}$ & $\begin{array}{l}0.00 \\
0.00 \\
0.00 \\
0.06 \\
0.00 \\
0.00 \\
0.00\end{array}$ \\
\hline $4-1$ & 00 & $\begin{array}{l}45.14 \\
48.34 \\
48.59 \\
49.16\end{array}$ & $\begin{array}{l}87 \\
66 \\
11 \\
55\end{array}$ & & $\begin{array}{l}1 \\
1 \\
1 \\
1\end{array}$ & & & $\begin{array}{r}10.30 \\
8.06 \\
9.27 \\
7.80 \\
1.64 \\
1.99\end{array}$ & $\begin{array}{l}0.35 \\
0.58 \\
0.52 \\
0.47\end{array}$ & & $\begin{array}{l}.09 \\
.00 \\
.00 \\
12 \\
.00\end{array}$ \\
\hline & $\begin{array}{l}0.00 \\
0.38\end{array}$ & $\begin{array}{l}45.57 \\
47.27 \\
47.43 \\
47.53\end{array}$ & $\begin{array}{l}3.59 \\
3.35\end{array}$ & & & & & 9.67 & & & $\begin{array}{l}.10 \\
.00 \\
.00 \\
.00 \\
.00 \\
.00\end{array}$ \\
\hline 1-2 & 0.00 & 52.76 & 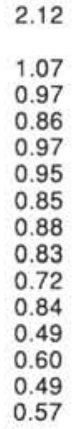 & $\begin{array}{l}1 \\
1 \\
1 \\
1 \\
1 \\
1 \\
1\end{array}$ & & & 7 & 1.27 & 5.68 & & 0 \\
\hline & & & & & & & & & & & $\begin{array}{l}0 \\
0\end{array}$ \\
\hline & & & & & & & & & & & \\
\hline $1-2$ & & & & & & & & $\begin{array}{l}2.11 \\
4.81\end{array}$ & $\begin{array}{l}1.71 \\
1.83 \\
3.57\end{array}$ & & 0.1 \\
\hline & & & & & $\begin{array}{r}10.8 \\
10.9 \\
7.0 \\
8.1\end{array}$ & & & & 4.8 & & $\begin{array}{l}0.22 \\
0.11 \\
0.22\end{array}$ \\
\hline & & & & & & & & & & & 0.27 \\
\hline
\end{tabular}


Appendix (continued).

AWl- $\mathrm{P}_{2} \mathrm{O}_{5} \quad \mathrm{SiO}_{2} \quad \mathrm{TiO}_{2} \quad \mathrm{Al}_{2} \mathrm{O}_{3} \quad \mathrm{FeO} \quad \mathrm{MnO} \quad \mathrm{MgO} \quad \mathrm{CaO} \quad \mathrm{K}_{2} \mathrm{O} \quad \mathrm{Na}_{2} \mathrm{O} \quad \mathrm{Cl}$

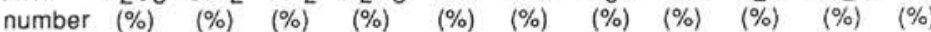

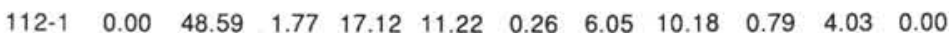
$\begin{array}{lllllllllllll}112-1 & 0.00 & 48.71 & 1.68 & 17.04 & 11.26 & 0.22 & 6.10 & 10.22 & 0.84 & 3.84 & 0.09\end{array}$ $\begin{array}{llllllllllll}112-1 & 0.00 & 48.74 & 1.82 & 17.21 & 10.93 & 0.20 & 5.99 & 10.34 & 0.85 & 3.86 & 0.06\end{array}$ $\begin{array}{llllllllllll}112-1 & 0.00 & 60.99 & 0.58 & 12.80 & 10.28 & 0.38 & 0.00 & 1.12 & 4.61 & 8.91 & 0.33\end{array}$ $\begin{array}{llllllllllll}112.1 & 0.00 & 61.14 & 0.73 & 12.84 & 10.33 & 0.37 & 0.00 & 1.18 & 4.61 & 8.27 & 0.37\end{array}$ $\begin{array}{lllllllllllll}112-1 & 0.00 & 74.31 & 0.18 & 11.94 & 2.86 & 0.19 & 0.00 & 0.30 & 5.05 & 4.82 & 0.34\end{array}$ $\begin{array}{llllllllllll}112-1 & 0.00 & 74.63 & 0.19 & 12.01 & 2.79 & 0.00 & 0.00 & 0.20 & 4.91 & 4.93 & 0.32\end{array}$

$\begin{array}{llllllllllll}112-2 & 0.00 & 45.17 & 3.08 & 16.30 & 11.85 & 0.27 & 6.14 & 12.86 & 1.12 & 3.21 & 0.00\end{array}$ $\begin{array}{llllllllllll}112-2 & 0.00 & 46.49 & 3.11 & 15.94 & 11.25 & 0.33 & 5.65 & 12.75 & 1.30 & 3.10 & 0.07\end{array}$ $\begin{array}{lllllllllllll}112-2 & 0.00 & 69.74 & 0.29 & 14.06 & 3.76 & 0.16 & 0.00 & 0.56 & 5.57 & 5.51 & 0.36\end{array}$ $\begin{array}{llllllllllll}112-2 & 0.00 & 74.33 & 0.18 & 11.46 & 3.08 & 0.00 & 0.00 & 0.26 & 4.90 & 5.17 & 0.62\end{array}$ $\begin{array}{llllllllllll}112-2 & 0.00 & 75.43 & 0.00 & 11.42 & 2.82 & 0.00 & 0.00 & 0.16 & 4.80 & 4.88 & 0.49\end{array}$ $\begin{array}{llllllllllll}112-2 & 0.00 & 75.98 & 0.23 & 11.83 & 2.46 & 0.00 & 0.00 & 0.30 & 4.67 & 4.30 & 0.23\end{array}$

$\begin{array}{llllllllllll}112-3 & 0.52 & 46.33 & 3.35 & 16.01 & 11.80 & 0.24 & 5.05 & 10.84 & 1.37 & 4.49 & 0.00\end{array}$ $\begin{array}{llllllllllll}112-3 & 0.46 & 46.44 & 3.29 & 16.40 & 11.82 & 0.25 & 5.02 & 10.81 & 1.37 & 4.16 & 0.00\end{array}$ $\begin{array}{llllllllllll}112-3 & 0.50 & 46.64 & 3.24 & 16.20 & 11.66 & 0.29 & 5.04 & 10.87 & 1.47 & 4.04 & 0.06\end{array}$ $\begin{array}{llllllllllll}112-3 & 0.26 & 46.70 & 3.42 & 16.03 & 11.88 & 0.30 & 4.93 & 10.87 & 1.34 & 4.27 & 0.00\end{array}$ $\begin{array}{lllllllllllll}112-3 & 0.43 & 47.01 & 3.30 & 15.89 & 11.88 & 0.16 & 5.11 & 10.88 & 1.38 & 3.97 & 0.00\end{array}$ $\begin{array}{llllllllllll}112-3 & 0.00 & 71.42 & 0.35 & 9.85 & 6.47 & 0.28 & 0.00 & 0.34 & 4.49 & 6.13 & 0.68\end{array}$

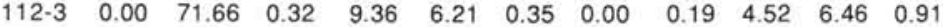

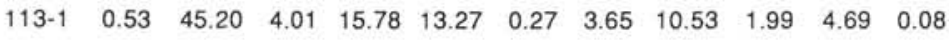

$\begin{array}{llllllllllll}113-2 & 0.17 & 50.37 & 3.79 & 14.53 & 12.96 & 0.29 & 4.12 & 8.77 & 1.29 & 3.71 & 0.00\end{array}$ $\begin{array}{llllllllllll}113-2 & 0.00 & 54.43 & 1.66 & 14.58 & 12.66 & 0.29 & 4.09 & 8.74 & 0.49 & 2.86 & 0.19\end{array}$ $\begin{array}{llllllllllll}113-2 & 0.00 & 55.04 & 0.88 & 17.04 & 9.66 & 0.29 & 4.27 & 9.06 & 0.35 & 3.33 & 0.07\end{array}$ $\begin{array}{rlllllllllll}113-2 & 0.00 & 55.79 & 0.99 & 15.87 & 11.01 & 0.26 & 3.94 & 8.98 & 0.44 & 2.59 & 0.13\end{array}$ $\begin{array}{llllllllllll}113-2 & 0.00 & 56.11 & 1.31 & 14.55 & 12.21 & 0.22 & 3.54 & 8.82 & 0.62 & 2.54 & 0.06\end{array}$

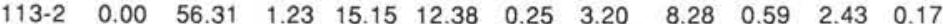
$\begin{array}{llllllllllll}113-2 & 0.00 & 57.80 & 1.46 & 16.91 & 9.11 & 0.24 & 0.67 & 4.61 & 3.86 & 5.21 & 0.12\end{array}$ $\begin{array}{llllllllllll}113-2 & 0.00 & 64.68 & 0.48 & 17.01 & 4.30 & 0.21 & 0.00 & 1.02 & 5.85 & 6.28 & 0.15\end{array}$

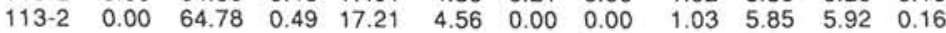

$\begin{array}{llllllllllll}113-4 & 0.00 & 55.10 & 1.10 & 16.03 & 12.25 & 0.19 & 3.40 & 8.69 & 0.59 & 2.64 & 0.00\end{array}$ $\begin{array}{lllllllllllll}113-4 & 0.00 & 55.31 & 0.96 & 15.55 & 11.26 & 0.18 & 4.29 & 9.05 & 0.53 & 2.73 & 0.14\end{array}$ $\begin{array}{llllllllllll}113-4 & 0.00 & 55.39 & 1.94 & 17.00 & 8.62 & 0.20 & 3.34 & 7.77 & 0.82 & 4.79 & 0.12\end{array}$

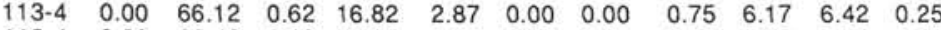
$\begin{array}{llllllllllll}113-4 & 0.00 & 66.48 & 0.00 & 15.95 & 4.38 & 0.00 & 0.00 & 0.52 & 5.00 & 7.24 & 0.43\end{array}$

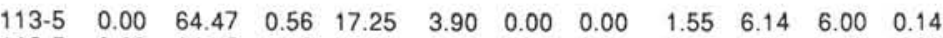
$\begin{array}{llllllllllll}113-5 & 0.00 & 64.75 & 0.36 & 16.34 & 4.68 & 0.20 & 0.00 & 1.14 & 5.59 & 6.73 & 0.20\end{array}$

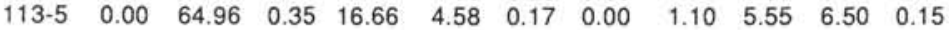

$\begin{array}{llllllllllll}114-1 & 0.00 & 66.59 & 0.64 & 15.96 & 3.30 & 0.24 & 0.00 & 0.66 & 5.90 & 6.46 & 0.24\end{array}$ $\begin{array}{llllllllllll}114-1 & 0.00 & 66.78 & 0.59 & 16.04 & 3.25 & 0.25 & 0.00 & 0.55 & 5.98 & 6.34 & 0.22\end{array}$ $\begin{array}{llllllllllll}114-1 & 0.00 & 67.06 & 0.50 & 15.86 & 3.28 & 0.24 & 0.00 & 0.56 & 5.84 & 6.46 & 0.20\end{array}$ $\begin{array}{llllllllllll}114-1 & 0.00 & 67.46 & 0.59 & 13.80 & 4.66 & 0.33 & 0.00 & 0.48 & 6.19 & 6.13 & 0.37\end{array}$ $\begin{array}{llllllllllll}114-1 & 0.00 & 74.47 & 0.22 & 12.42 & 2.85 & 0.00 & 0.00 & 0.29 & 4.66 & 4.82 & 0.27\end{array}$

$\begin{array}{llllllllllll}114-2 & 0.79 & 44.21 & 3.74 & 16.95 & 12.02 & 0.28 & 4.08 & 10.81 & 2.15 & 4.67 & 0.15\end{array}$ $\begin{array}{llllllllllll}114-2 & 0.61 & 44.41 & 4.22 & 16.17 & 14.50 & 0.27 & 4.64 & 9.27 & 1.24 & 4.32 & 0.09\end{array}$ $\begin{array}{llllllllllll}114-2 & 1.11 & 46.35 & 3.39 & 17.51 & 10.89 & 0.29 & 3.41 & 9.03 & 2.75 & 5.11 & 0.14\end{array}$ $\begin{array}{llllllllllll}114-2 & 0.54 & 46.48 & 2.90 & 18.01 & 10.99 & 0.26 & 3.28 & 8.96 & 3.03 & 5.20 & 0.13\end{array}$ $\begin{array}{llllllllllll}114-2 & 0.86 & 47.23 & 3.17 & 18.42 & 10.33 & 0.29 & 2.95 & 8.54 & 2.75 & 5.30 & 0.17\end{array}$ $\begin{array}{llllllllllll}114-2 & 0.00 & 61.19 & 0.46 & 19.12 & 3.75 & 0.00 & 0.00 & 1.14 & 6.53 & 7.56 & 0.25\end{array}$ $\begin{array}{llllllllllll}114-2 & 0.00 & 68.31 & 0.61 & 13.65 & 4.46 & 0.54 & 0.00 & 0.53 & 6.10 & 5.45 & 0.35\end{array}$

$\begin{array}{llllllllllll}114-5 & 0.00 & 47.08 & 2.36 & 16.66 & 11.90 & 0.23 & 6.54 & 10.90 & 0.65 & 3.67 & 0.00\end{array}$ $\begin{array}{llllllllllll}114-5 & 0.00 & 47.82 & 3.54 & 15.36 & 13.64 & 0.21 & 4.32 & 9.79 & 1.50 & 3.83 & 0.00\end{array}$ $\begin{array}{llllllllllll}114-5 & 0.90 & 51.16 & 2.38 & 15.55 & 13.04 & 0.27 & 2.14 & 6.41 & 2.76 & 5.24 & 0.14\end{array}$ $\begin{array}{llllllllllll}114-5 & 0.98 & 51.48 & 2.29 & 15.50 & 12.75 & 0.23 & 2.17 & 6.41 & 2.78 & 5.26 & 0.15\end{array}$

$\begin{array}{llllllllllll}115-2 & 0.00 & 63.07 & 0.74 & 16.46 & 5.65 & 0.26 & 0.00 & 1.67 & 6.16 & 5.91 & 0.07\end{array}$ $\begin{array}{llllllllllll}115-2 & 0.00 & 63.90 & 0.60 & 15.96 & 5.44 & 0.31 & 0.00 & 1.05 & 5.27 & 7.28 & 0.18\end{array}$ $\begin{array}{llllllllllll}115-2 & 0.00 & 63.95 & 0.51 & 15.63 & 5.74 & 0.27 & 0.00 & 1.09 & 5.40 & 7.26 & 0.15\end{array}$ $\begin{array}{llllllllllll}115-2 & 0.00 & 64.28 & 0.50 & 15.55 & 5.63 & 0.25 & 0.00 & 0.98 & 5.03 & 7.59 & 0.20\end{array}$ $\begin{array}{llllllllllll}115-2 & 0.00 & 64.29 & 0.44 & 15.71 & 5.66 & 0.36 & 0.00 & 0.90 & 5.07 & 7.38 & 0.18\end{array}$ $\begin{array}{llllllllllll}115-2 & 0.00 & 64.40 & 0.39 & 16.04 & 5.73 & 0.15 & 0.00 & 1.01 & 5.28 & 6.87 & 0.13\end{array}$ $\begin{array}{llllllllllll}115-2 & 0.00 & 64.45 & 0.34 & 14.65 & 6.37 & 0.30 & 0.00 & 0.68 & 4.91 & 8.02 & 0.27\end{array}$ $\begin{array}{llllllllllll}115-2 & 0.00 & 64.70 & 0.43 & 15.47 & 5.53 & 0.27 & 0.00 & 0.83 & 4.97 & 7.58 & 0.23\end{array}$ 
Appendix (continued).

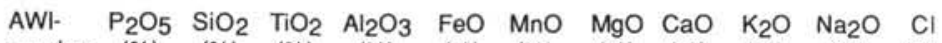

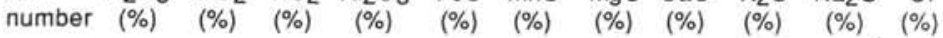
$\begin{array}{llllllllllll}115-3 & 0.00 & 44.22 & 3.73 & 16.35 & 14.00 & 0.27 & 5.39 & 11.25 & 0.93 & 3.73 & 0.00\end{array}$ $\begin{array}{lllllllllllll}115-3 & 0.00 & 44.69 & 3.34 & 16.85 & 12.87 & 0.24 & 6.34 & 10.95 & 0.87 & 3.85 & 0.00\end{array}$

$\begin{array}{lllllllllllll}115-3 & 0.97 & 44.95 & 3.46 & 18.36 & 10.38 & 0.25 & 3.76 & 10.48 & 2.33 & 5.00 & 0.08\end{array}$

$\begin{array}{llllllllllll}115-3 & 0.21 & 45.26 & 3.17 & 13.73 & 11.06 & 0.17 & 7.36 & 14.49 & 1.27 & 3.27 & 0.00\end{array}$

$\begin{array}{lllllllllllll}115-3 & 0.91 & 45.74 & 3.47 & 18.03 & 10.01 & 0.18 & 4.07 & 10.10 & 2.50 & 4.88 & 0.10\end{array}$

$\begin{array}{llllllllllll}115-3 & 0.89 & 45.99 & 3.17 & 17.86 & 10.53 & 0.25 & 3.61 & 9.60 & 2.65 & 5.32 & 0.13\end{array}$

$\begin{array}{llllllllllll}115-3 & 0.42 & 47.58 & 4.16 & 14.40 & 14.56 & 0.27 & 3.64 & 9.27 & 1.67 & 3.97 & 0.06\end{array}$

$\begin{array}{llllllllllll}115-3 & 0.77 & 47.93 & 3.67 & 14.62 & 13.37 & 0.26 & 3.96 & 8.93 & 1.87 & 4.32 & 0.11\end{array}$

$\begin{array}{llllllllllll}115-3 & 0.00 & 54.86 & 1.47 & 14.24 & 11.34 & 0.23 & 4.40 & 9.28 & 0.57 & 3.54 & 0.07\end{array}$

$\begin{array}{llllllllllll}115-3 & 0.00 & 58.45 & 1.38 & 15.63 & 9.92 & 0.42 & 2.13 & 7.17 & 1.02 & 3.73 & 0.14 \\ 115-3 & 0.00 & 59.06 & 1.25 & 16.89 & 9.36 & 0.00 & 1.59 & 7.20 & 0.79 & 3.74 & 0.12\end{array}$

$\begin{array}{llllllllllll}115-3 & 0.37 & 54.30 & 1.96 & 17.59 & 8.69 & 0.28 & 1.54 & 5.06 & 3.43 & 6.68 & 0.10\end{array}$

$\begin{array}{llllllllllll}115-5 & 1.01 & 42.52 & 3.51 & 14.84 & 12.55 & 0.24 & 4.37 & 12.24 & 2.56 & 5.97 & 0.19\end{array}$

$\begin{array}{llllllllllll}115-5 & 0.96 & 45.43 & 3.73 & 16.62 & 11.60 & 0.19 & 3.87 & 10.65 & 2.19 & 4.66 & 0.11\end{array}$

$\begin{array}{llllllllllll}115.5 & 1.10 & 45.74 & 3.35 & 16.73 & 11.58 & 0.20 & 4.17 & 9.77 & 2.26 & 5.01 & 0.11\end{array}$

$\begin{array}{llllllllllll}115-5 & 0.51 & 46.84 & 4.06 & 14.56 & 13.66 & 0.20 & 4.71 & 9.70 & 1.60 & 4.03 & 0.14\end{array}$

$\begin{array}{lllllllllllll}115-5 & 0.18 & 47.71 & 3.50 & 16.09 & 11.94 & 0.21 & 4.51 & 10.72 & 1.33 & 3.81 & 0.00\end{array}$

$\begin{array}{llllllllllll}116-1 & 0.76 & 47.76 & 3.69 & 15.19 & 13.75 & 0.32 & 3.73 & 8.33 & 1.90 & 4.48 & 0.09\end{array}$

$\begin{array}{llllllllllll}116-1 & 0.86 & 47.66 & 3.60 & 15.67 & 13.50 & 0.25 & 3.56 & 8.40 & 1.86 & 4.40 & 0.08\end{array}$

$\begin{array}{llllllllllll}116-2 & 0.00 & 64.40 & 0.77 & 16.14 & 5.15 & 0.17 & 0.00 & 1.46 & 6.06 & 5.84 & 0.00\end{array}$

$\begin{array}{llllllllllll}116-3 & 0.23 & 45.16 & 3.24 & 17.07 & 10.94 & 0.25 & 5.42 & 11.98 & 1.60 & 4.12 & 0.00\end{array}$

$\begin{array}{lllllllllllll}116-3 & 0.00 & 62.74 & 0.96 & 16.93 & 5.46 & 0.21 & 0.00 & 1.97 & 6.41 & 5.33 & 0.00\end{array}$

$\begin{array}{llllllllllll}116-3 & 0.00 & 62.90 & 0.41 & 17.29 & 4.95 & 0.25 & 0.00 & 1.25 & 5.53 & 7.17 & 0.25\end{array}$

$\begin{array}{llllllllllll}116-3 & 0.00 & 63.71 & 0.76 & 16.01 & 5.86 & 0.19 & 0.00 & 1.54 & 5.86 & 5.98 & 0.08\end{array}$

$\begin{array}{llllllllllll}116-4 & 0.37 & 45.69 & 2.82 & 17.21 & 12.25 & 0.22 & 6.54 & 10.45 & 0.96 & 3.50 & 0.00\end{array}$

$\begin{array}{lllllllllllll}116-4 & 0.35 & 45.70 & 2.90 & 17.20 & 12.06 & 0.16 & 6.66 & 10.33 & 0.91 & 3.73 & 0.00\end{array}$

$\begin{array}{llllllllllll}116-4 & 0.00 & 61.81 & 1.15 & 17.22 & 5.74 & 0.29 & 0.00 & 2.19 & 6.16 & 5.38 & 0.05\end{array}$

$\begin{array}{llllllllllll}116-4 & 0.00 & 61.98 & 1.15 & 17.10 & 5.47 & 0.27 & 0.00 & 2.18 & 6.38 & 5.47 & 0.00\end{array}$

$\begin{array}{llllllllllll}116-4 & 0.00 & 63.23 & 0.88 & 16.52 & 5.54 & 0.17 & 0.00 & 1.76 & 6.29 & 5.60 & 0.00\end{array}$

$\begin{array}{llllllllllll}116.4 & 0.00 & 65.64 & 0.58 & 15.38 & 5.34 & 0.28 & 0.00 & 1.11 & 5.49 & 6.10 & 0.08\end{array}$

$\begin{array}{llllllllllll}116-4 & 0.00 & 66.17 & 0.56 & 14.83 & 5.22 & 0.37 & 0.00 & 1.08 & 5.47 & 6.12 & 0.16\end{array}$

$\begin{array}{llllllllllll}116-4 & 0.00 & 67.17 & 0.50 & 14.20 & 5.15 & 0.17 & 0.00 & 0.80 & 5.33 & 6.49 & 0.18\end{array}$

$\begin{array}{llllllllllll}116-5 & 0.00 & 55.44 & 1.31 & 17.02 & 9.05 & 0.18 & 3.39 & 8.15 & 1.04 & 4.22 & 0.20\end{array}$

$\begin{array}{llllllllllll}116-5 & 0.00 & 61.74 & 1.17 & 17.37 & 5.78 & 0.27 & 0.00 & 2.21 & 6.14 & 5.31 & 0.00\end{array}$

$\begin{array}{llllllllllll}117-1 & 0.83 & 42.26 & 4.20 & 15.68 & 13.67 & 0.26 & 4.38 & 11.94 & 1.95 & 4.69 & 0.13\end{array}$

$\begin{array}{llllllllllll}117-1 & 0.79 & 42.62 & 4.36 & 15.82 & 14.09 & 0.27 & 4.57 & 11.38 & 2.11 & 3.92 & 0.08\end{array}$

$\begin{array}{llllllllllll}117-1 & 0.89 & 43.73 & 3.80 & 17.14 & 12.36 & 0.16 & 3.75 & 11.45 & 2.06 & 4.66 & 0.00\end{array}$

$\begin{array}{llllllllllll}117-1 & 0.99 & 43.74 & 4.03 & 16.34 & 13.02 & 0.35 & 3.87 & 11.03 & 2.26 & 4.22 & 0.15\end{array}$

$\begin{array}{llllllllllll}117-1 & 0.67 & 44.12 & 4.01 & 17.64 & 12.22 & 0.27 & 3.83 & 11.70 & 1.88 & 3.59 & 0.08\end{array}$

$\begin{array}{llllllllllll}117-1 & 0.40 & 52.58 & 1.81 & 18.41 & 9.41 & 0.17 & 1.65 & 5.78 & 3.28 & 6.36 & 0.14\end{array}$

$\begin{array}{llllllllllll}117-2 & 0.00 & 51.68 & 0.79 & 15.05 & 12.32 & 0.32 & 6.29 & 10.60 & 0.43 & 2.43 & 0.09\end{array}$

$\begin{array}{lllllllllllll}117-2 & 0.00 & 58.44 & 2.38 & 14.14 & 11.34 & 0.23 & 2.68 & 6.44 & 0.82 & 3.53 & 0.00\end{array}$

$\begin{array}{lllllllllllll}117-2 & 0.00 & 61.91 & 1.16 & 14.39 & 10.43 & 0.26 & 0.98 & 6.23 & 1.04 & 3.46 & 0.14\end{array}$

$\begin{array}{llllllllllll}117-3 & 1.04 & 42.17 & 3.15 & 15.06 & 11.24 & 0.34 & 4.75 & 13.69 & 2.58 & 5.76 & 0.23\end{array}$

$\begin{array}{llllllllllll}117-3 & 1.19 & 42.45 & 5.87 & 14.41 & 12.82 & 0.24 & 5.12 & 11.97 & 1.84 & 3.98 & 0.12\end{array}$

$\begin{array}{llllllllllll}117-3 & 0.97 & 43.19 & 2.70 & 17.24 & 11.52 & 0.27 & 3.77 & 11.81 & 2.56 & 5.76 & 0.23\end{array}$

$\begin{array}{lllllllllllll}117-3 & 0.65 & 43.90 & 3.12 & 18.23 & 11.68 & 0.20 & 3.67 & 10.59 & 2.45 & 5.19 & 0.20\end{array}$

$\begin{array}{llllllllllll}117-3 & 0.66 & 43.95 & 3.00 & 16.76 & 10.95 & 0.31 & 4.33 & 12.59 & 2.10 & 5.17 & 0.19\end{array}$

$\begin{array}{llllllllllll}117-3 & 0.34 & 44.40 & 2.86 & 16.89 & 10.60 & 0.37 & 5.12 & 12.64 & 2.08 & 4.54 & 0.16\end{array}$

$\begin{array}{lllllllllllll}117-3 & 0.43 & 44.57 & 3.11 & 17.79 & 11.00 & 0.22 & 4.13 & 11.52 & 2.16 & 4.95 & 0.12\end{array}$

$\begin{array}{llllllllllll}117-3 & 1.25 & 44.82 & 4.37 & 16.61 & 10.92 & 0.24 & 4.06 & 10.65 & 2.21 & 4.71 & 0.17\end{array}$

$\begin{array}{llllllllllll}117-3 & 1.22 & 44.83 & 4.46 & 16.39 & 10.82 & 0.29 & 4.11 & 10.64 & 2.24 & 4.85 & 0.16\end{array}$

$\begin{array}{llllllllllll}117-3 & 0.44 & 45.65 & 2.77 & 17.61 & 10.80 & 0.17 & 3.88 & 10.97 & 2.43 & 5.10 & 0.19\end{array}$

$\begin{array}{llllllllllll}117-3 & 0.80 & 46.87 & 3.85 & 15.74 & 11.81 & 0.30 & 4.15 & 10.13 & 1.99 & 4.24 & 0.11\end{array}$

$\begin{array}{llllllllllll}117-3 & 1.16 & 47.87 & 2.86 & 17.57 & 11.12 & 0.21 & 3.28 & 8.30 & 2.55 & 4.92 & 0.17\end{array}$

$\begin{array}{lllllllllllll}117-3 & 0.90 & 48.32 & 2.83 & 17.31 & 11.38 & 0.24 & 2.94 & 8.59 & 2.63 & 4.73 & 0.12\end{array}$

$\begin{array}{llllllllllll}117-4 & 0.77 & 43.80 & 4.01 & 16.82 & 11.21 & 0.34 & 4.25 & 11.55 & 2.04 & 5.14 & 0.07\end{array}$

$\begin{array}{lllllllllllll}117.4 & 0.87 & 50.15 & 3.28 & 16.00 & 11.85 & 0.34 & 2.44 & 6.75 & 2.97 & 5.25 & 0.10\end{array}$

$\begin{array}{lllllllllllll}117-4 & 0.00 & 61.24 & 0.26 & 18.82 & 4.36 & 0.20 & 0.00 & 1.33 & 6.35 & 7.18 & 0.26\end{array}$

$\begin{array}{lllllllllllll}117.4 & 0.00 & 62.29 & 0.58 & 18.33 & 4.26 & 0.00 & 0.00 & 1.56 & 6.44 & 6.39 & 0.16\end{array}$

$\begin{array}{llllllllllll}117-4 & 0.00 & 67.72 & 0.47 & 14.21 & 5.17 & 0.20 & 0.00 & 0.46 & 4.88 & 6.57 & 0.31\end{array}$

$\begin{array}{llllllllllll}117-5 & 0.59 & 47.41 & 2.84 & 18.27 & 10.25 & 0.26 & 2.78 & 8.16 & 2.87 & 6.32 & 0.25\end{array}$

$\begin{array}{lllllllllllll}117-5 & 0.90 & 47.62 & 2.73 & 19.44 & 9.76 & 0.27 & 1.97 & 7.71 & 2.73 & 6.62 & 0.24\end{array}$

$\begin{array}{llllllllllll}117-5 & 0.64 & 47.78 & 2.85 & 17.97 & 10.05 & 0.00 & 2.92 & 9.31 & 2.46 & 5.89 & 0.13\end{array}$

$\begin{array}{llllllllllll}117-5 & 0.80 & 48.15 & 2.60 & 18.34 & 9.67 & 0.23 & 2.48 & 8.25 & 2.85 & 6.39 & 0.24\end{array}$

$\begin{array}{llllllllllll}117.5 & 0.20 & 49.12 & 3.06 & 17.89 & 9.85 & 0.22 & 3.41 & 10.78 & 1.18 & 4.22 & 0.07\end{array}$

$\begin{array}{llllllllllllll}117-5 & 0.54 & 50.70 & 2.47 & 17.87 & 10.64 & 0.24 & 2.33 & 7.12 & 2.54 & 5.37 & 0.18\end{array}$

$\begin{array}{llllllllllll}117.5 & 0.00 & 67.42 & 0.63 & 16.07 & 3.36 & 0.22 & 0.00 & 0.50 & 6.38 & 5.28 & 0.15\end{array}$ 
Appendix (continued).

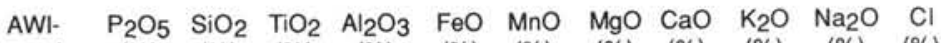

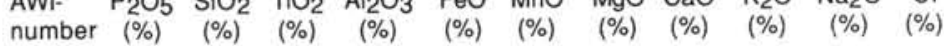

$\begin{array}{llllllllllll}118-1 & 0.26 & 45.08 & 3.71 & 16.23 & 12.20 & 0.19 & 4.96 & 12.04 & 1.54 & 3.72 & 0.06\end{array}$

$\begin{array}{llllllllllll}118-1 & 0.47 & 45.97 & 3.82 & 17.06 & 11.78 & 0.30 & 4.15 & 9.83 & 2.18 & 4.37 & 0.07\end{array}$

$\begin{array}{llllllllllll}118-1 & 0.51 & 46.17 & 4.00 & 17.13 & 11.79 & 0.22 & 4.11 & 9.72 & 2.11 & 4.16 & 0.08\end{array}$

$\begin{array}{llllllllllll}118-3 & 0.00 & 47.40 & 2.18 & 16.48 & 11.63 & 0.27 & 5.97 & 10.82 & 1.00 & 3.98 & 0.07\end{array}$

$\begin{array}{lllllllllllll}118-3 & 0.00 & 49.49 & 2.04 & 16.67 & 10.77 & 0.25 & 5.89 & 10.22 & 0.85 & 3.76 & 0.07\end{array}$

$\begin{array}{llllllllllll}118-3 & 0.00 & 49.60 & 2.03 & 16.38 & 11.46 & 0.00 & 5.45 & 10.34 & 0.84 & 3.90 & 0.00\end{array}$

$\begin{array}{llllllllllll}118-4 & 0.61 & 44.25 & 3.31 & 17.19 & 11.88 & 0.30 & 4.32 & 11.45 & 2.12 & 4.48 & 0.08\end{array}$

$\begin{array}{lllllllllllll}118-4 & 0.58 & 44.46 & 3.19 & 17.36 & 11.87 & 0.27 & 4.29 & 11.62 & 2.07 & 4.18 & 0.11\end{array}$

$\begin{array}{lllllllllllll}118-4 & 0.38 & 47.92 & 2.67 & 16.83 & 12.04 & 0.36 & 3.34 & 8.87 & 2.22 & 5.26 & 0.10\end{array}$

$\begin{array}{llllllllllll}118-5 & 0.00 & 48.48 & 1.72 & 17.22 & 11.13 & 0.25 & 6.31 & 10.05 & 0.80 & 4.05 & 0.00\end{array}$

$\begin{array}{llllllllllll}118-5 & 0.46 & 51.24 & 2.07 & 20.57 & 7.99 & 0.17 & 1.57 & 7.18 & 3.17 & 5.41 & 0.16\end{array}$

$\begin{array}{llllllllllll}119-2 & 0.00 & 46.90 & 2.32 & 16.59 & 11.53 & 0.23 & 6.42 & 10.41 & 1.00 & 4.59 & 0.00\end{array}$

$\begin{array}{lllllllllllll}119-2 & 0.00 & 47.18 & 2.15 & 16.53 & 11.80 & 0.20 & 6.35 & 10.41 & 1.00 & 4.29 & 0.07\end{array}$

$\begin{array}{llllllllllll}119-2 & 0.00 & 47.20 & 2.39 & 16.77 & 11.75 & 0.30 & 6.30 & 10.30 & 0.97 & 4.02 & 0.00\end{array}$

$\begin{array}{llllllllllll}119-2 & 0.00 & 47.22 & 2.26 & 16.53 & 11.48 & 0.24 & 6.54 & 10.28 & 1.00 & 4.25 & 0.06\end{array}$

$\begin{array}{lllllllllllll}119-2 & 0.00 & 47.36 & 2.26 & 16.58 & 11.69 & 0.17 & 6.41 & 10.50 & 0.98 & 4.06 & 0.00\end{array}$

$\begin{array}{llllllllllll}119-2 & 0.00 & 47.49 & 2.15 & 16.69 & 11.75 & 0.15 & 6.56 & 10.10 & 0.82 & 4.08 & 0.00\end{array}$

$\begin{array}{llllllllllll}119-2 & 0.00 & 48.43 & 1.96 & 16.92 & 10.84 & 0.20 & 6.47 & 10.22 & 0.97 & 3.89 & 0.10\end{array}$

$\begin{array}{llllllllllll}119-3 & 1.02 & 46.70 & 3.89 & 14.70 & 14.88 & 0.25 & 4.00 & 9.19 & 1.28 & 4.10 & 0.00\end{array}$

$\begin{array}{llllllllllll}119-3 & 0.00 & 66.64 & 0.63 & 14.78 & 5.52 & 0.36 & 0.00 & 1.67 & 5.17 & 5.07 & 0.16\end{array}$

$\begin{array}{llllllllllll}119-3 & 0.00 & 66.99 & 0.57 & 14.82 & 5.18 & 0.23 & 0.00 & 1.54 & 5.21 & 5.30 & 0.15\end{array}$

$\begin{array}{llllllllllll}119-3 & 0.00 & 69.73 & 0.33 & 14.09 & 3.99 & 0.13 & 0.00 & 1.13 & 5.41 & 5.06 & 0.13\end{array}$

$\begin{array}{llllllllllll}119-3 & 0.00 & 77.70 & 1.18 & 6.62 & 0.00 & 0.00 & 0.00 & 3.98 & 2.20 & 8.33 & 0.00\end{array}$

$\begin{array}{llllllllllll}146-1 & 0.51 & 45.10 & 3.41 & 17.96 & 10.06 & 0.27 & 3.98 & 10.57 & 2.67 & 5.33 & 0.13\end{array}$

$\begin{array}{lllllllllllll}146-1 & 0.57 & 45.47 & 3.39 & 17.97 & 9.57 & 0.16 & 4.14 & 10.70 & 2.71 & 5.19 & 0.12\end{array}$

$\begin{array}{llllllllllll}146-1 & 0.52 & 45.77 & 3.48 & 17.86 & 9.57 & 0.17 & 4.02 & 10.58 & 2.81 & 5.12 & 0.09\end{array}$

$\begin{array}{llllllllllll}146-1 & 0.63 & 46.09 & 3.31 & 18.24 & 9.44 & 0.18 & 3.62 & 10.18 & 2.80 & 5.36 & 0.17\end{array}$

$\begin{array}{lllllllllllll}146-1 & 0.19 & 46.30 & 2.92 & 16.51 & 11.33 & 0.31 & 5.16 & 11.69 & 1.27 & 4.24 & 0.08\end{array}$

$\begin{array}{llllllllllll}146-1 & 0.00 & 46.50 & 2.96 & 16.33 & 11.08 & 0.25 & 5.47 & 12.37 & 1.26 & 3.78 & 0.00\end{array}$

$\begin{array}{llllllllllll}146-1 & 0.74 & 46.60 & 3.89 & 16.05 & 12.37 & 0.24 & 3.88 & 9.67 & 1.68 & 4.83 & 0.06\end{array}$

$\begin{array}{llllllllllll}146-1 & 0.33 & 47.45 & 2.93 & 16.53 & 11.60 & 0.28 & 4.56 & 10.56 & 1.46 & 4.29 & 0.00\end{array}$

$\begin{array}{lllllllllllll}146-1 & 0.33 & 47.46 & 2.93 & 16.53 & 11.61 & 0.28 & 4.56 & 10.55 & 1.46 & 4.29 & 0.00\end{array}$

$\begin{array}{lllllllllllll}146-1 & 0.79 & 49.96 & 1.96 & 17.83 & 10.57 & 0.28 & 2.03 & 6.70 & 3.32 & 6.29 & 0.27\end{array}$

$\begin{array}{lllllllllllll}146-1 & 0.49 & 54.48 & 1.92 & 17.54 & 9.39 & 0.26 & 2.28 & 5.61 & 2.25 & 5.65 & 0.14\end{array}$

$\begin{array}{llllllllllll}146-1 & 0.00 & 75.53 & 0.00 & 12.02 & 2.37 & 0.00 & 0.00 & 0.32 & 5.27 & 4.22 & 0.28\end{array}$

$\begin{array}{llllllllllll}146-2 & 0.00 & 67.74 & 0.33 & 14.73 & 4.49 & 0.16 & 0.00 & 0.85 & 5.40 & 6.13 & 0.17\end{array}$

$\begin{array}{lllllllllllll}146-2 & 0.00 & 67.31 & 0.54 & 14.94 & 4.31 & 0.23 & 0.00 & 0.82 & 5.51 & 6.13 & 0.22\end{array}$

$\begin{array}{lllllllllllll}146-2 & 0.00 & 67.89 & 0.38 & 14.96 & 4.52 & 0.18 & 0.00 & 0.75 & 5.37 & 5.76 & 0.19\end{array}$

$\begin{array}{lllllllllllll}146-2 & 0.00 & 68.00 & 0.47 & 14.74 & 4.52 & 0.14 & 0.00 & 0.78 & 5.51 & 5.67 & 0.17\end{array}$

$\begin{array}{llllllllllll}146-3 & 0.58 & 46.90 & 3.69 & 16.32 & 12.73 & 0.32 & 4.67 & 9.21 & 1.44 & 4.08 & 0.06\end{array}$

$\begin{array}{lllllllllllll}146-3 & 0.00 & 47.23 & 2.29 & 16.07 & 13.08 & 0.31 & 6.24 & 11.16 & 0.49 & 2.98 & 0.00\end{array}$

$\begin{array}{lllllllllllll}146-3 & 0.00 & 61.62 & 0.69 & 11.95 & 10.49 & 0.33 & 0.00 & 1.19 & 4.64 & 8.60 & 0.30\end{array}$

$\begin{array}{lllllllllllll}146-3 & 0.00 & 61.62 & 0.69 & 11.95 & 10.49 & 0.33 & 0.00 & 1.19 & 4.64 & 8.60 & 0.30\end{array}$

$\begin{array}{llllllllllll}146-3 & 0.00 & 61.77 & 0.75 & 12.12 & 10.56 & 0.36 & 0.00 & 1.30 & 4.53 & 8.32 & 0.31\end{array}$

$\begin{array}{llllllllllll}146-3 & 0.00 & 61.78 & 0.67 & 12.19 & 10.58 & 0.37 & 0.00 & 1.22 & 4.59 & 8.31 & 0.29\end{array}$

$\begin{array}{lllllllllllll}146-3 & 0.00 & 61.87 & 0.65 & 12.13 & 10.62 & 0.36 & 0.00 & 1.19 & 4.50 & 8.41 & 0.28\end{array}$

$\begin{array}{lllllllllllll}146-3 & 0.00 & 61.87 & 0.65 & 12.13 & 10.62 & 0.36 & 0.00 & 1.19 & 4.50 & 8.41 & 0.28\end{array}$

$\begin{array}{lllllllllllll}146-3 & 0.00 & 61.88 & 0.71 & 16.12 & 7.59 & 0.33 & 0.00 & 2.10 & 5.31 & 5.86 & 0.09\end{array}$

$\begin{array}{llllllllllll}146-3 & 0.00 & 62.26 & 0.70 & 11.82 & 10.79 & 0.23 & 0.00 & 1.28 & 4.57 & 8.08 & 0.27\end{array}$

$\begin{array}{llllllllllll}146-3 & 0.00 & 62.36 & 0.59 & 12.19 & 10.33 & 0.32 & 0.00 & 1.22 & 4.68 & 8.02 & 0.30\end{array}$

$\begin{array}{llllllllllll}147-2 & 0.69 & 44.91 & 4.08 & 16.86 & 11.93 & 0.28 & 4.84 & 9.95 & 2.05 & 4.29 & 0.11\end{array}$

$\begin{array}{lllllllllllll}147-2 & 0.00 & 48.75 & 2.89 & 15.91 & 13.17 & 0.21 & 4.07 & 9.59 & 1.45 & 3.98 & 0.00\end{array}$

$\begin{array}{llllllllllll}147-2 & 0.00 & 48.97 & 3.08 & 15.61 & 13.02 & 0.25 & 3.94 & 9.65 & 1.43 & 3.99 & 0.06\end{array}$

$\begin{array}{rlllllllllll}147-2 & 0.00 & 49.07 & 2.66 & 15.94 & 12.47 & 0.28 & 4.26 & 10.27 & 1.32 & 3.73 & 0.00\end{array}$

$\begin{array}{llllllllllll}147-2 & 0.18 & 50.35 & 2.66 & 15.84 & 12.39 & 0.34 & 3.50 & 8.62 & 1.66 & 4.36 & 0.10\end{array}$

$\begin{array}{llllllllllll}147-2 & 0.00 & 50.93 & 2.62 & 15.92 & 12.39 & 0.28 & 3.36 & 8.61 & 1.71 & 4.19 & 0.00\end{array}$

$\begin{array}{lllllllllllll}147-2 & 0.00 & 52.13 & 2.34 & 15.84 & 12.00 & 0.32 & 2.92 & 8.08 & 1.97 & 4.40 & 0.00\end{array}$

$\begin{array}{lllllllllllll}147-2 & 0.00 & 52.62 & 2.60 & 15.78 & 11.42 & 0.31 & 3.05 & 7.98 & 2.02 & 4.01 & 0.08\end{array}$

$\begin{array}{llllllllllll}147-2 & 0.00 & 75.67 & 0.20 & 11.32 & 3.10 & 0.00 & 0.00 & 0.27 & 4.80 & 4.44 & 0.21\end{array}$ 

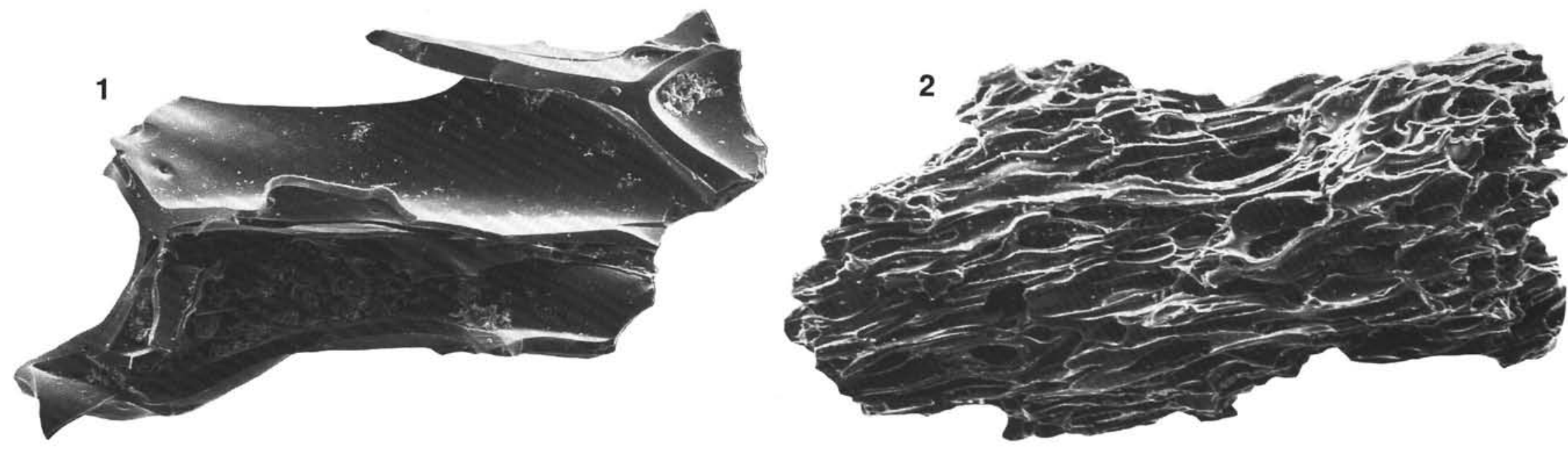

$0.1 \mathrm{~mm}$

$0.1 \mathrm{~mm}$
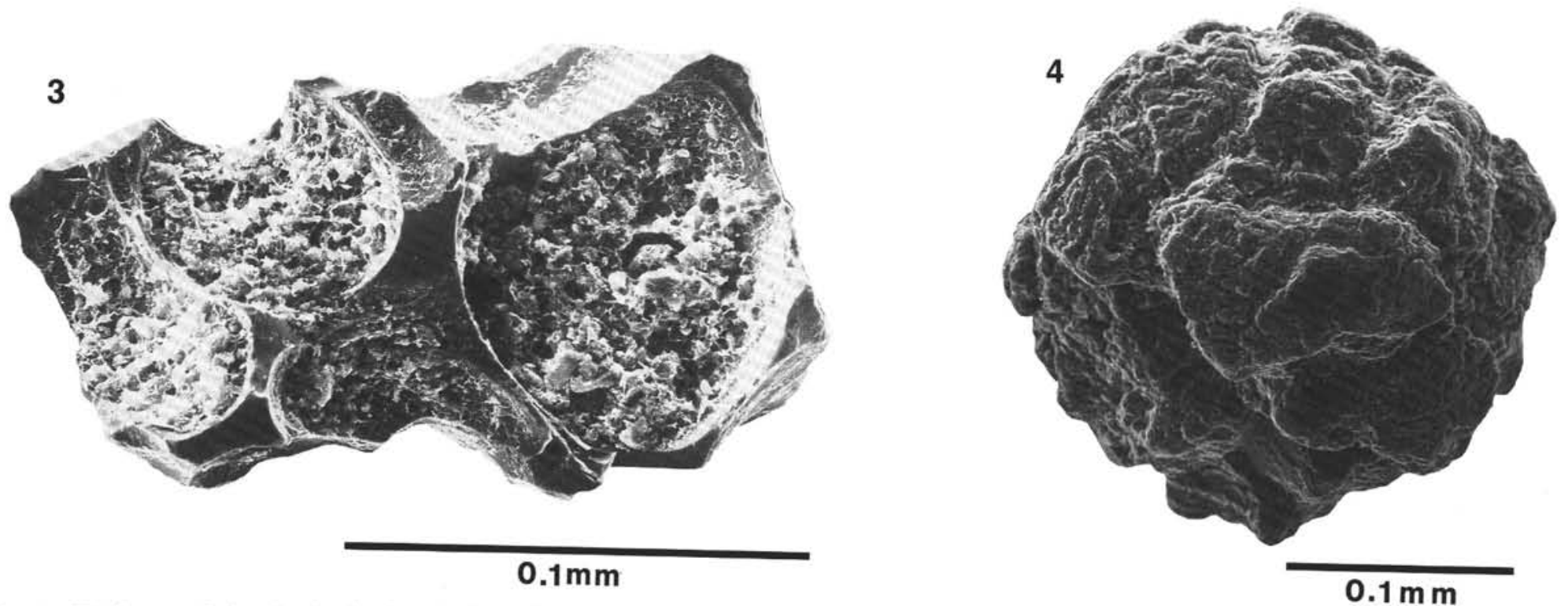

Plate 1. SEM images of glass shards, showing shard morphology and alteration of older ashes. Bar length is $0.1 \mathrm{~mm}$. 1. Fragment of elongated bubble cavities (Site 736). 2. Highly vesicular glass particle (Site 736). 3. Vesicular glass particle from an Oligocene tephra (Site 737). Glass alteration shown by vesicle filling with a secondary mineral. 4. Heavily altered blocky shaped glass
grain without vesicles (Site 737). 